\title{
The axial skeleton of Bagualia alba (Dinosauria: Eusauropoda) from the Early Jurassic of Patagonia
}

\author{
Kevin Leonel Gomez, Jose Luis Carballido, and Diego Pol
}

\begin{abstract}
Sauropod dinosaurs were the dominant large-bodied herbivores in many Mesozoic terrestrial ecosystems. Such predominance took place after a faunal replacement event linked to a global environmental change during the Early Jurassic (Pliensbachian-Toarcian), when the smaller basal sauropodomorphs were replaced by giant forms in the subclade Eusauropoda. Two main characteristics of this latter group are large body size (exceeding 10 tons) and a remarkably long neck. New data is presented on the axial anatomy of one of the earliest known eusauropods, Bagualia alba from the Toarcian levels of the Cañadón Asfalto Formation (Chubut Province, Argentina), which bear on the origins of these characteristics. Optimizing relevant axial characters on sauropodomorph phylogeny highlights important changes in cervical anatomy in the evolution of Sauropoda: a greater elongation of the neck due to both an increase in the vertebral count and the elongation of each cervical vertebra, and the appearance of pneumatic structures, first on the external surface (e.g., pleurocoels, laminae within the pleurocoels, accessory laminae of the neural arch) and subsequently invading the internal body of the vertebrae. These two changes have been considered essentials for body mass increase in Eusauropoda. The Toarcian age of Bagualia indicates that several important modifications in the axial skeleton and especially the neck occurred early in eusauropod evolution. These modifications are of ecomorphological importance and likely influenced the success of the eusauropods during the climatic changes surrounding the Pliensbachian-Toarcian crisis.
\end{abstract}

Kevin Leonel Gomez. Consejo Nacional de Investigaciones Científicas y Técnicas, Museo Paleontológico Egidio Feruglio, Fontana 140, 9100, Trelew, Argentina. kgomez@mef.org.ar

Jose Luis Carballido. Consejo Nacional de Investigaciones Científicas y Técnicas, Museo Paleontológico Egidio Feruglio, Fontana 140, 9100, Trelew, Argentina. jcarballido@mef.org.ar

Diego Pol. Consejo Nacional de Investigaciones Científicas y Técnicas, Museo Paleontológico Egidio Feruglio, Fontana 140, 9100, Trelew, Argentina.dpol@mef.org.ar

Keywords: Sauropoda; Argentina; cervical vertebrae; body size evolution

Submission: 5 July 2021. Acceptance: 18 November 2021.

Gomez, Kevin Leonel, Carballido, Jose Luis, and Pol, Diego. 2021. The axial skeleton of Bagualia alba (Dinosauria: Eusauropoda) from the Early Jurassic of Patagonia. Palaeontologia Electronica, 24(3):a37. https://doi.org/10.26879/1176

palaeo-electronica.org/content/2021/3490-axial-skeleton-of-bagualia

Copyright: December 2021 Society of Vertebrate Paleontology.

This is an open access article distributed under the terms of the Creative Commons Attribution License, which permits unrestricted use, distribution, and reproduction in any medium, provided the original author and source are credited.

creativecommons.org/licenses/by/4.0 


\section{INTRODUCTION}

Sauropods were giant, herbivorous dinosaurs that predominated in many Mesozoic ecosystems (Upchurch et al., 2004). However, our understanding of the early evolution and diversification of Sauropoda is poor, due to the scarce fossil record prior to the Middle Jurassic (Allain and Aquesbi, 2008; McPhee et al., 2015). Sauropods from the Early Jurassic are known from Africa (e.g., Cooper, 1984; Allain and Aquesbi, 2008; McPhee et al., 2014, 2015; Nicholl et al., 2018), Europe (e.g., Upchurch and Martin, 2002; Stumpf et al., 2015), India (e.g., Kutty et al., 2007; Bandyopadhyay et al., 2010), China (e.g., He et al., 1988; Li et al., 2010), and South America (e.g., Cúneo et al., 2013). The records of sauropods from the EarlyMiddle Jurassic from South America come from Central Patagonia (Chubut Province, Argentina) and currently include four taxa: Amygdalodon pata- gonicus Cabrera 1947, from the Cerro Carnerero Formation (Toarcian-Bajocian; Rauhut, 2003), and Volkheimeria chubutensis Bonaparte 1979, Patagosaurus fariasi Bonaparte 1979, and Bagualia alba Pol et al., 2020 from the Cañadón Asfalto Formation (Figure 1), currently dated as ToarcianBajocian (Cúneo et al., 2013). In recent analyses, Amygdalodon and Volkheimeria have been recovered as non-eusauropod sauropods, while Patagosaurus and Bagualia are early eusauropods (Carballido and Pol, 2010; Becerra et al., 2017; Holwerda and Pol, 2018; Mannion et al., 2019a; Pol et al., 2020). The Cañadón Asfalto Formation not only preserves a good record of late Early Jurassic sauropods, but also evidences floral and faunal changes (Cúneo et al., 2013; Figari et al., 2015) potentially reflecting global climatic changes linked to a Pliensbachian-Toarcian volcanic event

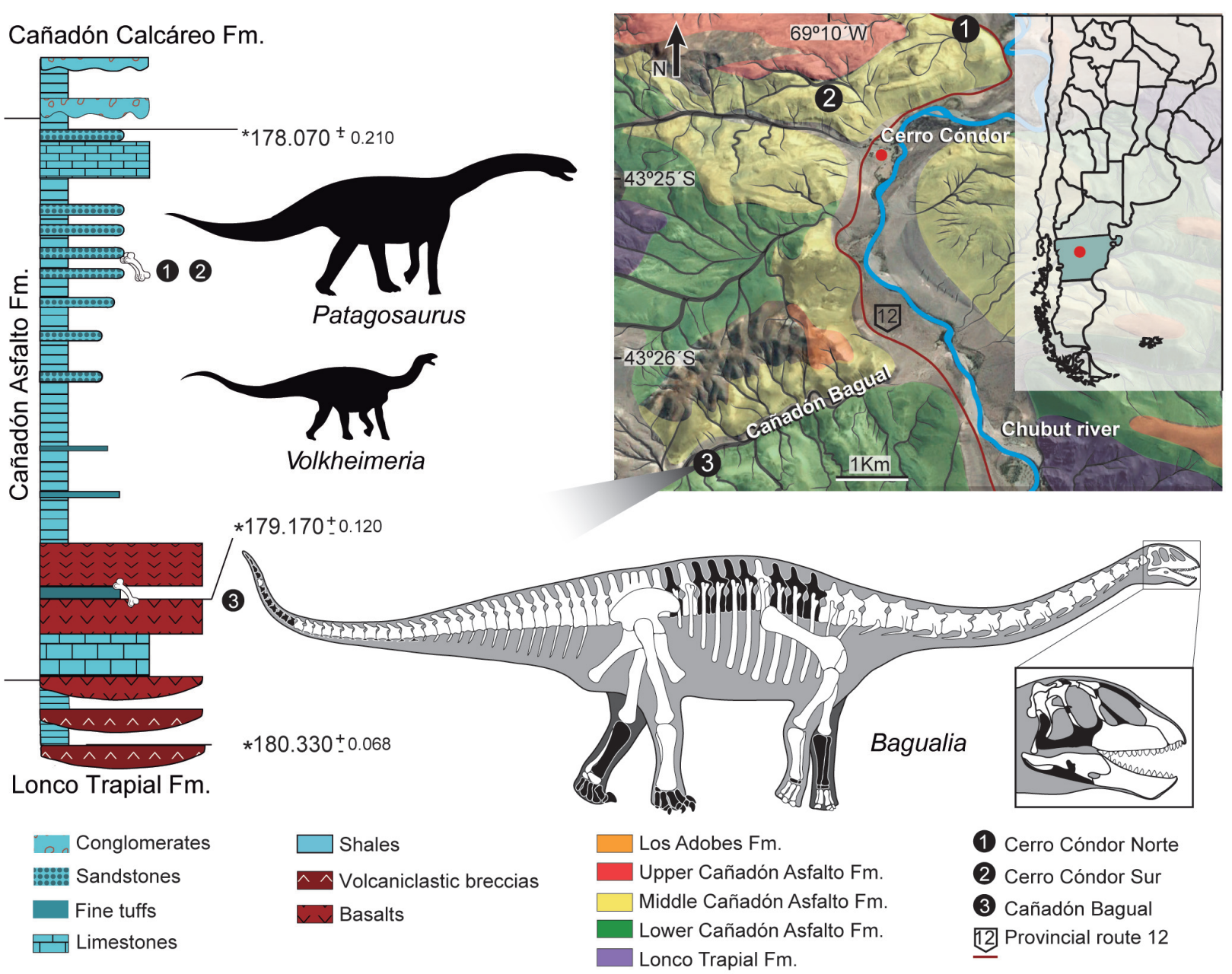

FIGURE 1. Location map and stratigraphic distribution of the main sauropods of the Cañadón Asfalto Formation, and reconstructed skeleton of Bagualia alba. Preserved bones in white and missing elements in black. Stratigraphy modified from Pol et al. (2020) and Figari (2005). Map data: Google, (C2021 CNES/Airbus, Landsat/ Copernicus, Maxar Technologies, Map data (C2021 
(180-185 Ma; Hesselbo et al., 2000; Sell et al., 2014; Burges et al., 2015).

The early evolution of sauropods was characterized by an increase in body size, elongation of the neck, and a transition from bipedal to quadrupedal posture (Wilson, 2002; Rauhut et al., 2011). The length of the sauropod neck exceeds that of all other known animals (Wedel, 2006) and is a hallmark change in the evolution of the group, allowing them not only to reach greater browsing heights but also to cover a wider feeding area without the need to move their massive bodies (Sander et al., 2011; Barrett, 2014). Elongation of the neck would have occurred through a combination of three morphological changes over the course of sauropod evolution: 1) appearance of new elements in the cervical region; 2) conversion of a dorsal vertebra and its incorporation into the cervical region; and 3) elongation of pre-existing cervical vertebrae (Upchurch, 1994; Wilson and Sereno, 1998). Recent studies on neck development in archosaurs, based on embryonic stages of crocodiles and birds (Mansfield and Abzhanov, 2010; Böhmer et al., 2019; Bui and Larsson, 2021), can provide insights on the evolution of sauropodomorph necks (Böhmer et al., 2015), but require corroboration with fossil data. Only a few basal sauropods preserve nearly complete axial series (e.g., Shunosaurus, Spinophorosaurus; Zhang, 1988; Remes et al., 2009), so our knowledge about the early evolution of neck elongation at the base of Sauropoda (i.e., the sequence of appearance of the three changes in neck anatomy listed above) is still limited. Relevant to this issue is the recent discovery of a new basal eusauropod from the Cañadon Asfalto Formation (Bagualia alba) represented by abundant axial material. The sediments that buried Bagualia have been dated with high precision (U$\mathrm{Pb}$ ID-TIMS at $179.12 \pm 0.24 \mathrm{Ma}$; Toarcian), making it the earliest definite record of Eusauropoda and marking the onset of eusauropod predominance in terrestrial ecosystems (Pol et al., 2020). Therefore, its cervical anatomy can inform the sequence of anatomical changes occurring during the rise of Eusauropoda.

The axial skeleton of Bagualia is described in detail and compared not only with those taxa recorded in the same formation (i.e., Patagosaurus and Volkheimeria), but also with other phylogenetically proximate taxa (basal, i.e., non-neosauropod, sauropods). Based on an expanded version of our recent phylogenetic analysis, we corroborate the position of Bagualia as a basal eusauropod closely related to Spinophorosaurus and Patagosaurus.
We use the phylogenetic trees obtained in this study to optimize certain axial characters related to lengthening of the neck to reevaluate key changes in sauropod evolution.

\section{Institutional Abbreviations}

AMNH, American Museum of Natural History, New York, U.S.A.; BYU, Brigham Young University, Museum of Paleontology, Provo, U.S.A.; CM, Carnegie Museum of Natural History, Pittsburgh, U.S.A.; CPT, Museo de la Fundación Conjunto Paleontológico de Teruel-Dinópolis, Aragón, Spain; GCP, Grupo Cultural Paleontológico de Elche, Museo Paleontológico de Elche, Spain; GMNH, Gunma Museum of Natural History, Gunma, Japan; IVPP, Institute of Vertebrate Paleontology and Paleoanthropology, Beijing; LCM, Leicester City Museums, Leicester; LFGT, Bureau of Land and Resources of Lufeng Country, Yunnan, China; MACN, Museo Argentino de Ciencias Naturales Bernardino Rivadavia, Buenos Aires, Argentina; MB, Museum für Naturkunde, Leibniz-Institut für Evolutions- und Biodiversitätsforschung, Berlin, Germany; MLP, Museo de La Plata, Buenos Aires, Argentina; $\mathbf{M M C H}$; Museo Municipal "Ernesto Bachmann", Villa el Chocón, Neuquén, Argentina; MNN, Musée National du Niger, Niamey, Republic of Niger; MPEF, Museo Paleontológico Egidio Feruglio, Chubut, Argentina; MUCPv, Museo de la Universidad del Comahue-Patagonia, Argentina; NHMUK, Natural History Museum, London, United Kingdom; NM, National Museum, Bloemfontein, South Africa; NMB, Staatliches Naturhistorisches Museum Braunschweig, Germany; PMU, Palaeontological Museum, Uppsala, Sweden; PVL, Fundacion Miguel Lillo, Universidad Nacional de Tucumán, San Miguel de Tucumán, Argentina; PVSJ, Instituto y Museo de Ciencias Naturales, San Juan, Argentina; TMM, Vertebrate Paleontology Laboratory, Austin, U.S.A.; ZDM, Zigong Dinosaur Museum, Zigong, China.

\section{Anatomical Abbreviations}

acdl, anterior centrodiapophyseal lamina; acpl, anterior centroparapophyseal lamina; al, accessory lamina; avk, anterior ventral keel; $\mathbf{c p}$, capitulum; cpof, centropostzygapophyseal fossa; cpol, centropostzygapophyseal lamina; cprl, centroprezygapophyseal lamina; cr, caudal recess; dp, diapophysis; epi, epipophysis; eprl, epipophysealprezygapophyseal lamina; hypa, hypantrum; hypo, hyposphene; Icpol, lateral centropostzygapophyseal lamina; mcpol, medial centropostzygapophyseal lamina; mdcpol, medial division of the 
centropostzygapophyseal lamina; mdcprl, medial division of the centroprezygapophyseal lamina; nc, neural canal; ppdI, paradiapophyseal lamina; pp, parapophysis; ped, pedicels; pl, pleurocoel; pcdl, posterior centrodiapophyseal lamina; posl, postspinal lamina; poz, postzygapophysis; podl, postzygodiapophyseal lamina; prsl, prespinal lamina; prz, prezygapophysis; prdl, prezygodiapophyseal lamina; prepi, pre-epipophysis; pvk, posterior ventral keel; stpol, single intrapostzygapophyseal lamina; stprl, single intraprezygapophyseal lamina; spol, spinopostzygapophyseal lamina; sprl, spinoprezygapophyseal lamina; str, striations; tb, tuberculum; tpol, intrapostzygapophyseal lamina; tprl, intraprezygapophyseal lamina.

\section{MATERIALS AND METHODS}

\section{Materials}

The studied material comprises axial elements (cervical, dorsal, sacral, and caudal vertebrae) both from the holotype and referred specimens of Bagualia alba (see below for details, and Appendix 1 for tables of measurements). Fossae and laminae are described following the nomenclature of Wilson et al. (2011) and Wilson (1999), respectively. We use the term pleurocoel to refer to a deep lateral depression of the centrum with well-defined edges, where the posterior could be weakly marked, as defined by Carballido and Sander (2014).

\section{Methods}

Elongation of cervical vertebrae. The elongation of the cervical vertebrae was calculated according to the Elongation Index (EI) sensu Wedel et al. (2000) as the anteroposterior length of the centrum divided by the midline height of the posterior articular face. In the case of highly deformed elements such as those with both lateromedial and dorsoventral centra crushed, the average of the width and height of the posterior articular face was used to calculate the El (El sensu Chure et al., 2010).

Source of comparative data. Comparisons with other sauropodomorphs made in the description and discussion were based on the published literature, photographs, and personal observations on certain taxa (see Table 1 in Appendix 1). The comparisons are primarily focused on non-neosauropod sauropodomorphs and some representative taxa of the main groups within Neosauropoda.

Phylogenetic analysis. The phylogenetic analysis was carried out using a modified version of the data matrix presented by Pol et al. (2020). The changes in the data matrix include score modifications for some species, expansion of the character sampling (incorporating 90 characters), removal of some characters and taxa, and modification in some character definitions (see Phylogenetic Analysis in Appendix 1). Thus, the resulting matrix is composed of a total of 104 taxa and 583 characters (Appendix 2 in .nex format). The phylogenetic analysis was carried out using an equal weights search in TNT (Goloboff et al., 2008).

\section{SYSTEMATIC PALEONTOLOGY}

DINOSAURIA Owen, 1842

SAURISCHIA Seeley, 1887

SAUROPODA Marsh, 1878

EUSAUROPODA Upchurch, 1995

Bagualia alba Pol, Ramezani, Gomez, Carballido, Paulina Carabajal, Rauhut, Escapa, and Cúneo, 2020

Figures 1-26

Holotype. MPEF-PV 3301 includes a posterior half of a skull articulated with seven cervical vertebrae. The material comprises: left lacrimal (MPEF-PV 3301/11), left prefrontal (MPEF-PV 3301/6), both frontals fused (MPEF-PV 3301/3), left parietal (MPEF-PV 3301/8), right postorbital (MPEF-PV 3301/10a,b), right and left squamosals (MPEF-PV 3301/9 and MPEF-PV 3301/7, respectively), right quadrate (MPEF-PV 3301/4), braincase (MPEF-PV 3301/1-2), one right proatlas (MPEF-PV 3301/5), atlas (MPEF-PV 3301/18), and cervical vertebrae from the axis (second) to the seventh cervical element (MPEF-PV 3301/12-16).

Referred specimens. All referred materials were found at the same locality as the holotype. These elements were recovered from a bonebed in which multiple individuals were found in association with isolated theropod teeth and a theropod dentary. Sauropod materials represent a minimum number of three individuals, based on the presence of three right humeri. The specimens are similarly sized (repeated humeri varying up to $15 \%$ in length). The repeated elements lack anatomical differences, leading us to consider this bonebed as a monospecific assemblage. Except for the articulated elements of the holotype, most other elements found at the excavation are catalogued as single specimens, as no obvious association or articulation can be established, although few specimens are composed of some articulated elements. The materials recovered provide an extensive sample of the anatomy of Bagualia (Figure 1) and include: left premaxilla (MPEF-PV 3305), two left maxillae (MPEF-PV 3304, 3341a), right maxilla 

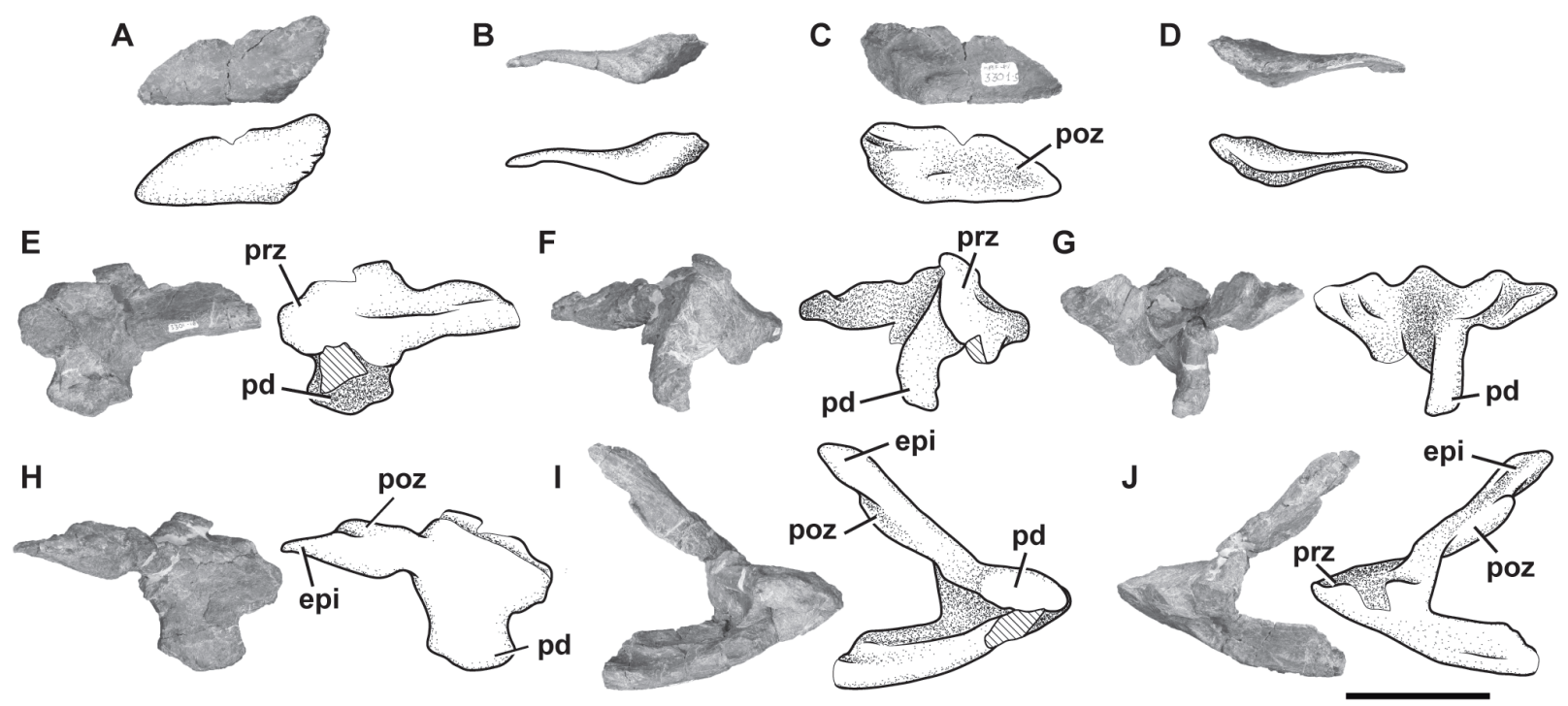

FIGURE 2. Proatlas and atlas of Bagualia alba. A-D, right proatlas (MPEF-PV3301/5) in lateral (A), ventral (B), medial (C) and dorsal (D) views; E-J, atlas (MPEF-PV 3301/18) in left lateral (E), anterior (F), posterior (G), right lateral $(\mathbf{H})$, ventral (I), and dorsal (J) views. Abbreviations: epi, epipophysis; pd, pedicel; poz, postzygapophysis; prz: prezygapophysis. Hatched pattern represents broken surfaces. Scale bar equals $5 \mathrm{~cm}$.

(MPEF-PV 3204), right nasal (MPEF-PV 3340), left quadrate (MPEF-PV3342), both dentaries and right surangular (MPEF-PV 3202), right surangular (MPEF-PV 3339), left pterygoid (MPEF-PV 11017), several isolated teeth (MPEF-PV 3146, 3174-3176, $3203,3205,3207-3209,11030-11039,11041-$ 11047, 11050), two middle cervical vertebrae (MPEF-PV 11040), mid-posterior cervical vertebra (MPEF-PV 3408), cervical centrum (MPEF-PV 3327), posterior cervical vertebrae (MPEF-PV 3348, 3349), anteriormost dorsal vertebra (MPEFPV 11023), four dorsal centra (MPEF-PV $3343,3403,3405,11012$ ), dorsal neural arch (MPEF-PV 11027), posterior dorsal vertebra (MPEF-PV 11000), two posterior dorsal neural arches with sacrum, both ilia, right pubis and the first ten caudal vertebrae articulated (MPEF-PV 11011), two segments of three articulated anterior caudal vertebrae (MPEF-PV 3316, 11044), 29 isolated caudal vertebrae (MPEF-PV 3179, 3300, $3314,3315,3317-3326,3328-3331,3344-3346$, $3389,3401,3402,3404,3406,3407,3409$, 11026), cervical ribs (MPEF-PV 11052), dorsal ribs (MPEF-PV 11058), five isolated caudal transverse processes (MPEF-PV 11001-11005), 13 haemal arches (MPEF-PV3351-3359, 3390, 11009, 11010, 11025), two coracoids (MPEF-PV3387, 11015), three scapulae (MPEF-PV 3382-3384), two scapulocoracoids (MPEF-PV 3385, 3386), five humeri (MPEF-PV 3311,3338,3380,3381,11020), radius (MPEF-PV 3313), two ulnae (MPEF-PV 3312,
3379), ilium (MPEF-PV 3369), three pubes (MPEFPV 11011, 11019, 11051), two ischia (MPEF-PV 3337, 11016), five femora (MPEF-PV 3303, 3371, 11024, 11021, 11022), tibia (MPEF-PV 3374), two fibulae (MPEF-PV 3306, 3376), two astragalus (MPEF-PV 3307, 3308), calcaneum (MPEF-PV 11018), four metapodials (MPEF-PV 3309, 33323334/), four non-ungual phalanges (MPEF-PV $3335,11028,11029,11049$ ), and two ungual phalanges (MPEF-PV 3310, 3410).

Geographic occurrence. Bagual Canyon, $5 \mathrm{~km}$ south of Cerro Cóndor, Chubut, Argentina (Figure 1).

Stratigraphic occurrence. Lower levels of the Cañadón Asfalto Formation, dated at $179.17 \pm$ $0.12 \mathrm{Ma}$, Early Jurassic, Toarcian (Pol et al., 2020). Emended diagnosis. To the original diagnosis of Bagualia alba (see Pol et al., 2020) we added 11 characters (new characters are indicated by ${ }^{+}$and autapomorphies are marked by *): *pointed process on the anteroventral end of the premaxilla and anterodorsal end of the dentary; *anterior margin of the premaxilla without a marked step; *orbital margin of the prefrontal with a close 'V-shape' pointed medially, resulting in a short contribution to the orbit; *supretemporal fenestra about as anteroposteriorly long as lateromedially wide; *strongly marked proatlantal facets on the laterodorsal margin of the foramen magnum; +foramen magnum vertically taller than transversely wide (different to wider than tall foramen magnum present in Spino- 
phorosaurus and Nebulasaurus); ${ }^{*}$ concave ventral margin of the distal portion of the cultriform process; axis with the anterior process in the dorsal part of the neural spine (convergent in Jobaria and Europasaurus); *accessory lamina below the PCDL in middle cervical vertebrae; EPRL present in middle cervical vertebrae; ${ }^{+*}$ cervical vertebrae with an accessory lamina below the EPRL within the SDF; +dorsal centra without pleurocoels (different from eusauropods and derived taxa, in which pleurocoels are present); +dorsal vertebrae with single prespinal lamina (shared with Omeisaurus, Mamenchisaurus, Turiasauria, and several neosauropods); +middle and posterior dorsal centrum slightly dorsoventrally compressed with a height/ width ratio between 0.8 and 1 (shared with Cetiosaurus, Omeisaurus, and some neosauropods); +subcircular posterior dorsal neural canal (different from the slit-shaped canal of Patagosaurus and Spinophorosaurus); +PRDL in anteriormost caudal vertebrae (shared with Tazoudasaurus and absent in Spinophorosaurus and Patagosaurus); 'tubercle above the transverse process of the anterior and middle caudal vertebrae (present in Mamenchisaurus, Losillasaurus, and some neosauropods); +radial condyle of the humerus undivided on its anterior face (different from the divided condyle of other basal eusauropods); +tibia with a proximal condyle expanded transversely (shared with mamenchisaurids, Mierasaurus, and some neosauropods); +foramina at the base of the ascending process (different from most eusauropods and neosauropods).

\section{DESCRIPTION}

\section{Cervical Vertebrae}

A total of 12 cervical vertebrae were recovered in the quarry, of which the first seven cervical vertebrae plus one proatlas pertain to the holotype specimen (MPEF-PV 3301/5, /18, /12-16) whereas the other five are considered different specimens because no clear association was found between them (MPEF-PV 3327, MPEF-PV 3348; MPEF-PV 3349, MPEF-PV 3408, MPEF-PV 11040). The holotype specimen provides anatomical information for the anterior and middle section of the cervical series. Although the exact position of each referred specimen cannot be known, the specimens described herein provide information on the anterior to the posteriormost cervical vertebrae, such that combined they provide a nearly complete understanding of neck anatomy in Bagualia.
The description proceeds in an anterior-posterior direction, firstly describing in detail the holotype, followed by the referred specimens. One specimen, composed of two cervical vertebrae (MPEF-PV 11040), overlaps with the holotype and is briefly described after it. All cervical vertebrae are opisthocoelous (a synapomorphy of Amygdalodon and more derived taxa; Carballido and Sander, 2014) with both articular faces as wide as tall. They have elongate centra (with an elongation index between 2 and 4.6), a ventral keel, and marked pleurocoels. The lamination is well developed (see Table 2 in Appendix 1), as in all sauropods more derived than Vulcanodon (Wilson, 1999).

Proatlas. A single right proatlas (MPEF-PV 3301/ 5 ) was recovered from the quarry. In lateral view the proatlas is robust and rhomboidal in shape (Figure 2A-D), as in Adeopapposaurus (Martínez, 2009, figure 14), but differing from the subtriangular proatlas present in Leyesaurus (Apaldetti et al., 2011, figure $6 \mathrm{~A}$ ) and the gracile and elongate proatlas of Camarasaurus (Madsen et al., 1995, figure 50). This element becomes thinner posteriorly and thicker anteriorly, where it is medially flat and laterally convex, acquiring a D-shape in cross section. The anterior surface is flat, slightly beveled medially and oriented anterodorsally. In Camarasaurus, the anterior articular surface is not visible in medial view (Madsen et al., 1995, figure 50). This surface contacts the exoccipital and supraoccipital along the lateral and dorsal margin of the foramen magnum, respectively. Near the dorsal end of the anterior surface there is a short, anterodorsally oriented groove. The anteroventral edge is rounded and bulbous. The anteriormost end (anterodorsal margin) is thinner than the ventral one and is above the level of the dorsal margin of the postzygapophysis (Figure 2C). In contrast, Leyesaurus has an anterior end located in the middorsoventral length of the main body of the proatlas, below the dorsal margin of the postzygapophysis (Apaldetti et al., 2011, figure 6A). This margin is straight and probably contacts (or nearly so) the other proatlas to form the roof of the neural canal (Apaldetti et al., 2011). The lateral surface of the anterior margin of the proatlas is rough with short, thin striations oriented anteroposteriorly. In lateral view, the ventral margin is straight and the dorsal margin of the postzygapophysis is convex, posteroventrally oriented, and ends in a sharp point. The sharp posterior end of the postzygapophysis is also seen in some basal sauropodomorphs (e.g., Riojasaurus, Adeoppaposaurus; Bonaparte and 
Pumares, 1995, figure 5C-D; Martínez, 2009, figure 14). The lateral surface of the postzygapophysis is convex, and the medial one is slightly concave for the articulation with the prezygapophysis of the atlas.

Atlas. Only the neurapophyses of the atlas is present, which were clearly not fused to the intercentrum and with the right one completely preserved (MPEF-PV 3301/18; Figure 2E-J). The neural arch is anteroposteriorly elongated, approximately two times longer than high (measured from the anterior end of the prezygapophysis to the posterior end of the postzygapophysis). As in other sauropods, the neurapophyses are wing-shaped and posterodorsally directed (Figure 2E). Both neurapophyses appear anterodorsally fused, an uncommon morphology, since in sauropods both neurapophyses remain, in general, separated from each other as independent units (Harris, 2006). We interpret the neurapophyseal dorsal fusion as a consequence of diagenetic processes instead of the natural condition of the vertebra. Whereas the left pedicel is broken, the right one is nearly complete. Although the distal articular surface of the pedicel is slightly damaged, it can be seen that it was somewhat Vshaped in lateral view, as in other sauropodomorphs (e.g., Leyesaurus, Apaldettiet al., 2011, figure 6D), albeit open at a greater angle than that of other sauropodomorphs, such as Thecodontosaurus (Yates, 2003, figure 10C), Europasaurus (Carballido and Sander, 2014, figure 1), and Rapetosaurus (Curry Rogers, 2009, figure 6). In other sauropodomorphs the form of the articular surface of the pedicel is variable, having a rounded shape as in Adeopapposaurus (Martínez, 2009, figure 14A) or a flat shape as in Anchisaurus (Galton, 1976, figure 15A-B).

The prezygapophyses are anteriorly extended (around $2.4 \mathrm{~cm}$ ) from the anterior margin of the pedicel, unlike the anteroposteriorly short prezygapophyses of other sauropodomorphs, which are located posteriorly relative to the pedicel (e.g., Adeopapposaurus; Leyesaurus, Europasaurus, Camarasaurus, Apatosaurus; Apaldetti et al., 2011, figure 6D; Martínez, 2009, figure 14A; Carballido and Sander, 2014, figure 1H; GMNH 101; Gilmore, 1936, figure 6).

The postzygapophyses are well developed, with the articular surfaces having an elliptical shape with its longest axis anteroposteriorly oriented along the process. As in Adeopapposaurus and Leyesaurus, the prominent epipophyses are posteriorly directed from the mid-height of the postzygapophyses and end in a thin tip (preserved in the right neurapophysis; Figure $2 \mathrm{H}-\mathrm{J}$ ). The right complete epipophysis is separated from the articular surface of the postzygapophysis by a long deep groove, which extends posteriorly around $2 \mathrm{~cm}$ from the posterior margin of the articulation. Therefore, the epipophysis is almost as long anteroposteriorly as the postzygapophysis, being relatively shorter than that of Thecodontosaurus (Yates, 2003, figure 10C).

Axis. The preserved axis (MPEF-PV 3301/13) has been distorted by lateromedial compression (Figure 3). Its El is 2.3 (see Table 3 in Appendix 1) similar in proportions to other taxa (e.g., Tazoudasaurus, Patagosaurus; Allain et al., 2004, figure 8A-B; MACN-CH 936). In contrast, other eusauropods have lower Els (e.g., Cetiosaurus, Omeisaurus, Jobaria; Upchurch and Martin, 2002, figure 2A; He et al., 1988, figure 18; MNN TIG 35 ). The centrum has very deep lateral fossae (pleurocoels sensu Carballido and Sander, 2014) separated by a thin bony plate (approximately $1 \mathrm{~mm}$ thick). Whereas the anterior, dorsal, and ventral margin of the pleurocoel is well marked, the posterior margin is subtle, as in Tazoudasaurus (Allain and Aquesbi, 2008, figure 8A-B). This morphology is clearly different from that observed in several sauropods, which have pleurocoels completely bounded by sharp margins (e.g., Cetiosaurus, Patagosaurus, Jobaria; Upchurch and Martin, 2002, figure 2A; Holwerda et al., 2021; Sereno et al., 1999, figure 3, MNN TIG 3-5). As in most sauropods, the pleurocoel of Bagualia is simple instead of divided by an oblique lamina, contrasting with the divided pleurocoels present in derived eusauropods such as Omeisaurus (He et al., 1988, figure 18). In lateral view, the ventral margin of the centrum is slightly concave throughout its anteroposterior length. On the ventral surface of the centrum there is a ventral keel, which is slightly marked in the anterior portion and seems to become higher and stouter towards the posterior margin, though this is probably due to taphonomic deformation (Figure 2E). A ventral keel is a common feature present in basal sauropodomorphs (e.g., Sarahsaurus, Yizhousaurus, Leyesaurus; Marsh and Rowe, 2018, Zhang et al., 2018; Apaldetti et al., 2011), and is retained in basal sauropods and eusauropods (e.g., Amygdalodon, Tazoudasaurus, Spinophorosaurus, Patagosaurus, Jobaria; Rauhut, 2003; Allain and Aquesbi, 2008; Remeset al., 2009; Holwerda et al., 2021; MNN TIG3) and few neosauropods (e.g., Dicraeosaurus, Demandasaurus; Upchurch et al., 2004; Torcida et al., 2011). In some sauropodomorphs, the keel is 

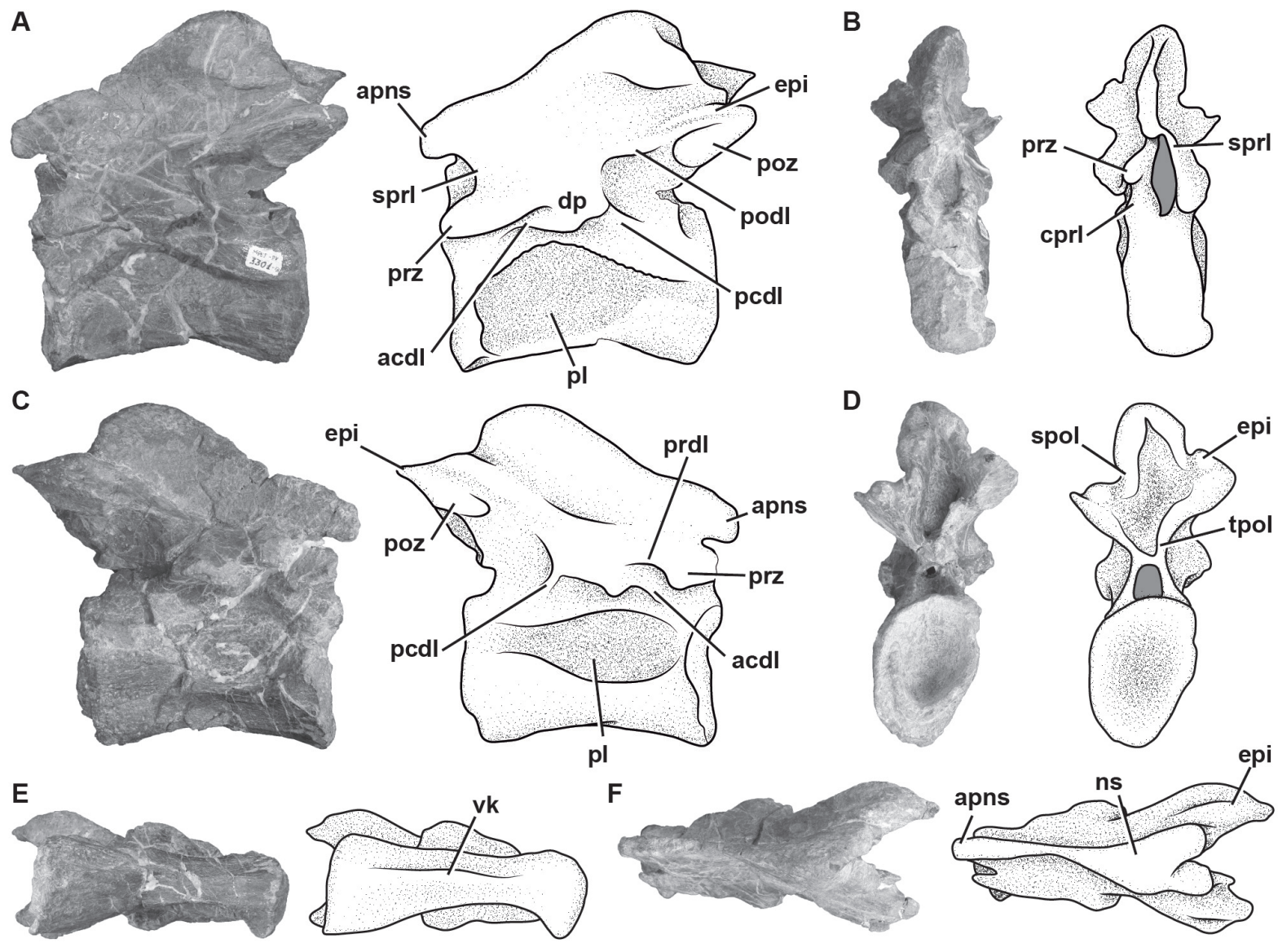

FIGURE 3. Axis of Bagualia alba (MPEF-PV3301/13) in left lateral (A), anterior (B), right lateral (C), posterior (D), ventral (E), and dorsal (F) views. Abbreviations: acdl, anterior centrodiapophyseal lamina; apns, anterior process of the neural spine; cprl, centroprezygapophyseal lamina; dp, diapophysis; epi, epipophysis; ns, neural spine; pcdl, posterior centrodiapophyseal lamina; podl, postzygodiapophyseal lamina; poz, postzygapophysis; prdl, prezygodiapophyseal lamina; prz, prezygapophysis; spol, spinopostzygapophyseal lamina; sprl, spinoprezygapophyseal lamina; tprl, intraprezygapophyseal lamina; vk, ventral keel. Scale bar equals $10 \mathrm{~cm}$.

absent in the anterior and middle cervical vertebrae (e.g., Riojasaurus, Plateosaurus, Lessemsaurus; Apaldetti et al., 2011), while in others it is found in all elements of the neck including the axis (e.g., Sarahsaurus, Yizhousaurus, Adeoppaposaurus, Leyesaurus; Marsh and Rowe, 2018, Zhang et al., 2018; Martínez, 2009; Apaldetti et al., 2011). In the basal sauropodomorph Yunnanosaurus, the keel appears from the third cervical onwards (Lu et al., 2007), while in Xingxiulong it appears between the fourth and ninth vertebra (e.g., Wang et al., 2017), and in Thecodontosaurus it is only observed in the tenth (i.e., the last vertebra of the neck; Yates, 2003). In the basal eusauropod Cetiosaurus, a ventral keel is present in vertebrae 1-3 (LCM G468.1968) and in cervical 12 (Upchurch and Martin, 2002). The restricted presence of a ventral keel is also observed in Jobaria, where it is present in the third cervical and from the ninth onwards (Upchurch and Martin, 2003). Although the parapophyses are poorly preserved in Bagualia, they are noticeably smaller than those of Patagosaurus (Bonaparte, 1986, figure 35) and are positioned differently, being in the mid-dorsoventral length of the centrum and directed lateroposteriorly from the anterior articular surface of the centrum.

The neural arch occupies the full anteroposterior length of the centrum, and there is a marked neurocentral suture, which would indicate that the axis belongs to a subadult individual (Carballido and Sander, 2014). The prezygapophyses are anteroposteriorly short and smooth (Figure 3B), and do not extend beyond the anterior articular face of the centrum. A thin PRDL runs from the prezygapophysis to the diapophysis (Figure $3 \mathrm{C}$ ), 
as is also observed in other sauropods including Patagosaurus (Bonaparte, 1986, figure 35).

The postzygapophyses are sheet-like with the long axis oriented anteroposteriorly and facing lateroventrally, so they are visible in lateral view, unlike Tazoudasaurus (Allain and Aquesbi, 2008) in which the postzygapophyses are more horizontally oriented. The postzygapophyses are better developed than the prezygapophyses and have a crest-shaped epipophysis on their dorsal surface, which extends about $0.5 \mathrm{~cm}$ from their posterior margin (Figure 3A, C). The TPOL (Wilson, 1999; Janensch, 1929; Osborn and Mook, 1921) is short and joins the postzygapophyses forming the roof of the neural canal, whereas the CPOLs are absent (Figure 3D). The transverse processes are well developed and anteroposteriorly short. In contrast to Tazoudasaurus, the ACDL is present and larger than the PCDL, which does not reach to the posterior margin of the articular surface of the centrum. The presence of centrodiapophyseal laminae is shared with other sauropods, such as Patagosaurus (Bonaparte, 1986, figure 35).

In lateral view, the neural spine is slightly concave anteroposteriorly, similar to but not so deep as that of Omeisaurus (He et al., 1988, figure 18B), Cetiosaurus (Upchurch and Martin, 2002, figure 2A), and Mamenchisaurus (Young and Zhao, 1972), and different from the flat or convex neural spine present in Tazoudasaurus (Allain and Aquesbi, 2008, figure 8B), Patagosaurus (Bonaparte, 1986, figure 35), and Jobaria (MNN TIG 35). The SPRLs are narrow and form the roof of the neural canal. In the anterior distal end of the neural spine, just above the junction of both the SPRLs, there is an anterior process (apns) that is also observed in the basal sauropodomorph Anchisaurus (Galton, 1976, figure 15D), the eusauropod Jobaria (MNN TIG 3-5), and a few neosauropods (i.e., Europasaurus, Erketu; Carballido and Sander, 2014, figure 2; Ksepka and Norell, 2006, figure 3). The basal sauropodomorph Sarahsaurus also has this process, but only in the middle and posterior cervical vertebrae, and also has a distal posterior projection of the spine (Marsh and Rowe, 2018). Therefore, the presence of an apns seems to be a character that was convergently acquired by various sauropodomorphs, both basal and derived. This process is considered as a part of the PRSL by Tschopp et al. (2015: character 151), which is described by Carballido and Sander (2014) in Europasaurus. The distal portion of the neural spine becomes broad (Figure 3F), and on its dorsal surface there are pits and grooves. In contrast with
Patagosaurus (Bonaparte, 1986), in Bagualia, the posterior end of the neural spine does not extend past the posterior articular surface of the centrum.

Third cervical vertebra. This vertebra (MPEF-PV $3301 / 12$ ) is completely preserved, but it is deformed at an angle of 45 degrees towards the right side, with respect to the longitudinal axis, so that the right apophyses and zygapophyses are above the level of the left ones by around $2 \mathrm{~cm}$ (Figure 4). The centrum has an El of 3.6 (see Table 3 in Appendix 1). The ventral surface is flat and bears a keel that occupies the anterior half of the anteroposterior length of the centrum and fades posteriorly (Figure 4E). A single ventral keel is present in many sauropodomorphs (e.g., Adeopapposaurus, Amygdalodon, Tazoudasaurus, Patagosaurus; Martínez, 2009; Rauhut, 2003; Allain and Aquesbi, 2008; Holwerda et al., 2021) with a few exceptions that have a forked keel (e.g., Spinophorosaurus, Barapasaurus; GCP-CV-4229; Bandyopadhyay et al., 2010). On the lateral sides there are well-marked pleurocoels that extend anteroposteriorly throughout almost the entire length of the centrum (Figure 4A, C). The septum that separates the left and right pleurocoels is very narrow, and like the axis, presents a rough lateral texture, and does not communicate with internal chambers or with other pneumatic cavities. Rough surfaces are typical of immature specimens (Varricchio, 1997), indicating fast-growing tissue (e.g., Marpmann et al., 2015), which in the case of the holotype specimen of Bagualia also accords with the visible neurocentral suture on its cervical vertebrae preserved. The centrum has well-developed ovoid-shaped parapophyses. As in the axis, the ventral margin of the pedicels of the neural arch is easily recognized (on both sides) due to the presence of the neurocentral suture, which has a rough and zipper-like shape. No dorsal notch on the margin of the posterior articular surface is present. This notch has been described as an autapomorphy of Europasaurus (Sander et al., 2006), but it is also present in other camarasauromorphs such as Giraffatitan (MB.R.2181) and Euhelopus (PMU 233) (Carballido and Sander, 2014). In lateral view, at the base of the neural arch, there is a U-shaped recess which was previously considered an autapomorphy of Spinophorosaurus (Remes et al., 2009). The transverse processes are well developed. The complete right diapophysis (the articular surface for the tuberculum of the cervical rib) is ellipticallyshaped as in other sauropods (e.g., Tazoudasaurus, Patagosaurus; Allain and Aquesbi, 2008, figure 9A-B; Holwerda et al., 2021, figure 4). 

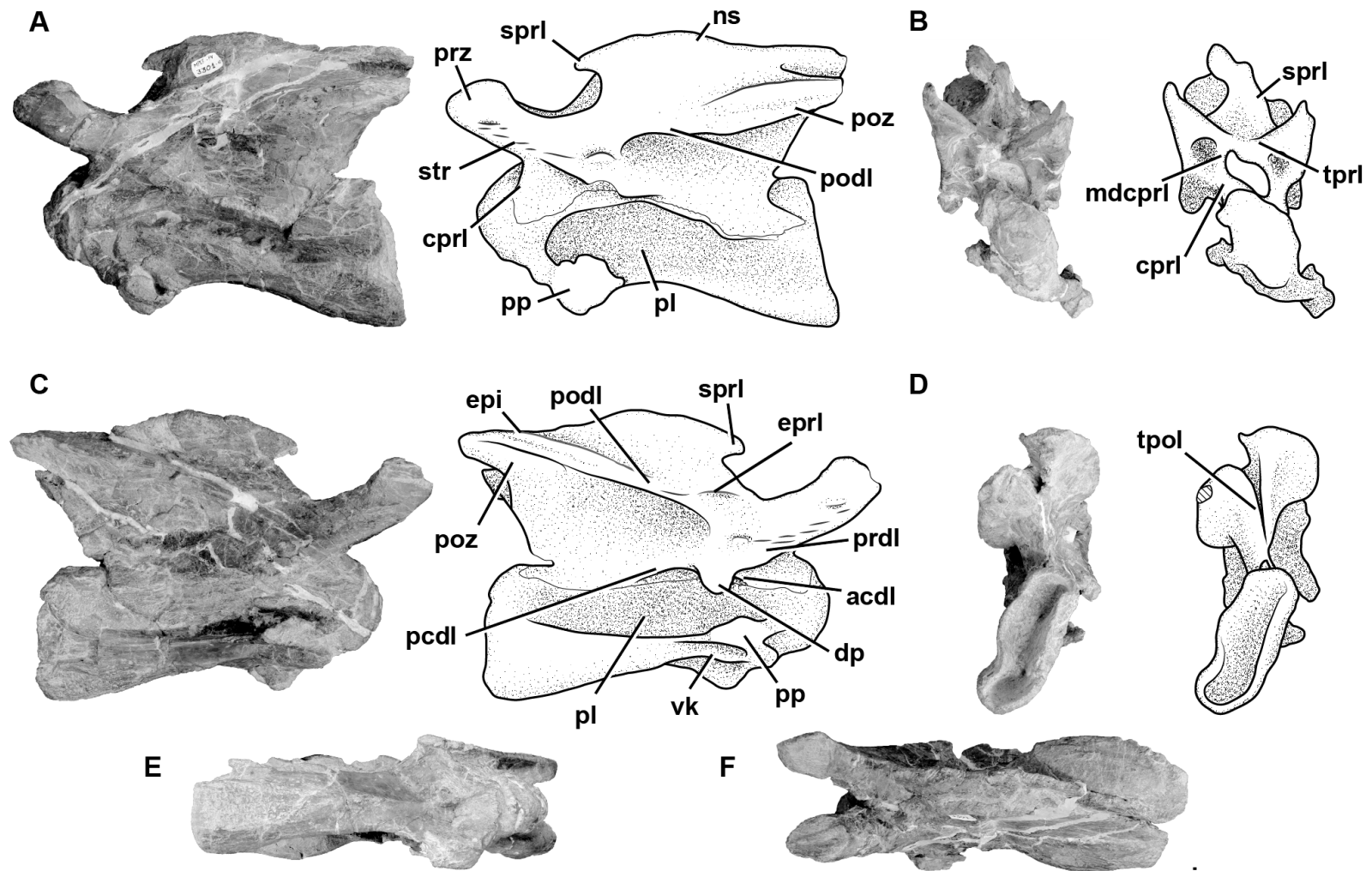

E
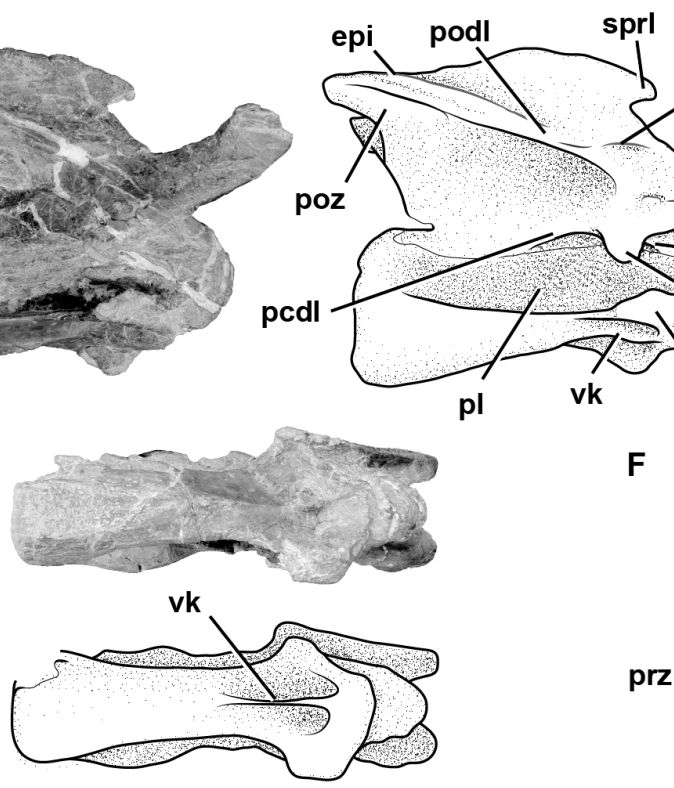

eprl

D
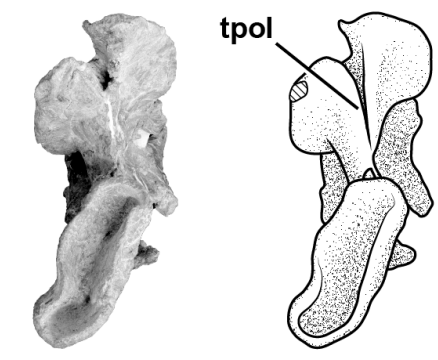

pl

vk
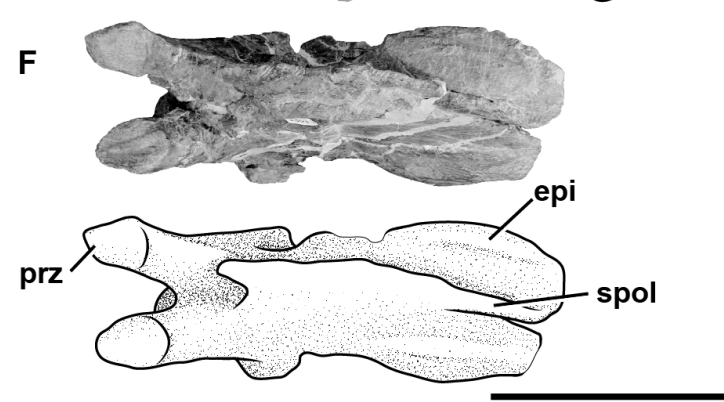

FIGURE 4. Third cervical vertebra of Bagualia alba (MPEF-PV 3301/12) in left lateral (A), anterior (B), right lateral (C), posterior (D), ventral (E), and dorsal (F) views. Abbreviations: acdl, anterior centrodiapophyseal lamina; cprl, centroprezygapophyseal lamina; cr, caudal recess; dp, diapophysis; epi, epipophysis; eprl, epipophyseal-prezygapophyseal lamina; mdcprl, medial division of the centroprezygapophyseal lamina; ns, neural spine; pcdl, posterior centrodiapophyseal lamina; pl, pleurocoel; podl, postzygodiapophyseal lamina; poz, postzygapophysis; pp, parapophysis; prdl, prezygodiapophyseal lamina; prz, prezygapophysis; spol, spinopostzygapophyseal lamina; sprl, spinoprezygapophyseal lamina; str, striations; tpol, intrapostzygapophyseal lamina; tprl, intraprezygapophyseal lamina; vk, ventral keel. Hatched pattern represents broken surfaces. Scale bar equals $10 \mathrm{~cm}$.

On the lateral surface of the prezygapophyseal process, on both right and left sides, there are numerous small horizontal smooth striations (Figure $4 \mathrm{~A}, \mathrm{C})$. These extend to the transverse process, staying close to the anterior border of the PRDL. At the dorsal base of the transverse process, a marked horizontal bulge develops, which could be related to the aforementioned striations. On the right lateral surface, a low EPRL is present (Figure 4C). This lamina is better developed in the middle and posterior vertebrae (see below).

The PCDL extends at an angle of about $10^{\circ}$ from the diapophysis to nearly contact the posterior articular surface of the centrum, as in Jobaria (MNN TIG 3-5) and Spinophorosaurus (GCP-CV
4229). The ACDL is shorter than the PCDL but reaches the anterior margin of the centrum. These laminae define a CDF below the transverse process, which is weakly marked. The neural canal is anteriorly semicircular; posteriorly it is crushed dorsoventrally and displaced to the left side due to the general deformation of the vertebra. The prezygapophyses are well preserved, and their articular surfaces are flat and lateral surfaces convex. Both articular surfaces of the prezygapophyses are directed dorsolaterally and surpass the anterior articular surface of the centrum by around $2 \mathrm{~cm}$. This feature is also observed in other sauropods (e.g., Spinophorosaurus, Jobaria; GCP-CV 4229, MNN TIG 3-5). On the lateral surface of the prezy- 
gapophyseal process, close to the anteroventral margin, there is an anteroposteriorly oriented short, low crest. This small crest does not contact the CPRL and could be homologous to the pre-epipophyses (sensu Wilson and Upchurch, 2009; see below). The CPRLs are short and are dorsally divided. Only the medial ramus of the CPRL (mdCPRL) contacts the TPRL and defines a deep CPRF (Wilson et al., 2011; Figure 4B), as in most sauropods (e.g., Spinophorosaurus, Europasaurus; GCP-CV 4229; Carballido and Sander, 2014). The TPRLis short and contacts directly with the neural canal, forming the roof of it (Figure 4B). The PRDL is well developed (especially on the right side) and is running from the lateroventral edge of the prezygapophyseal process up to the anterior margin of the diapophysis. As in most sauropods the prezygapophyses are flat and surpass the articular surface of the centrum, in the case of this element by around $2 \mathrm{~cm}$. The postzygapophyses are above to prezygapophyses, as in other sauropods (Bonaparte, 1999; McPhee et al., 2015). Due to deformation, the right postzygapophysis is approximately $2 \mathrm{~cm}$ higher than the left one. As in most sauropodomorphs, on the laterodorsal surface of the postzygapophyses there are tall, discrete epipophyses (Figure 4F). However, in some basal sauropodomorphs the epipophyses are flat and low (e.g., Thecodontosaurus; Yates, 2003). The epipophysis in Bagualia runs posteriorly and surpasses the posterior margin of the postzygapophysis as in other sauropodomorphs (e.g., Thecodontosaurus, Patagosaurus, Spinophorosaurus, Turiasaurus, Jobaria, Euhelopus; Yates, 2003; PVL 4170; Remes et al., 2009; CPT 1220; MNN TIG 35; Wilson and Upchurch, 2009). The epipophyses run separately from the SPOL and do not reach it, although this may be related to the position of the vertebra in the cervical series (see below). The postzygapophyses of this vertebra have a greater angle than in the axis, resulting in a long TPOL (Figure 4D). The TPOL is long and extends downwards to the neural canal through a short STPOL. Therefore, unlike in the axis, the dorsal margin of the neural canal ceases to be in contact with the TPOL. The CPOLs are considered absent in this vertebra, as no clear lamina can be traced, except for the thin walls of the neural canal as in Tazoudasaurus (Allain and Aquesbi, 2008, figure 9l-J). The PODL are very well developed, running from the diapophysis to the laterodorsal margin of the postzygapophyseal facet. Below the PODL there is a deep, triangular POCDF, which is more elongated than the one present in the axis.
The neural spine is non-bifurcated and five times anteroposteriorly longer than lateromedially wide, and its anteroposterior length is about $43 \%$ of the centrum length. The neural spine is low, with its dorsal edge slightly higher than the postzygapophyses. Its width does not vary throughout its anteroposterior length. Its height is half the height of the centrum (at the posterior articular surface). The dorsal edge of the neural spine is almost flat, with a horizontal edge. The anterior half of the dorsal margin is slightly convex, and the posterior one slightly concave. Spinal laminae (SPRL, SPOL) are thin and enclose deep fossae (SPRF, SPOF). In the anterior distal end of the neural spine, both SPRLs extend anteriorly, producing a process in lateral view. Both SPRLs contacting each other dorsally (maybe a result of preservation), so the SPRF is enclosed by them dorsally. This morphology is also present in the middle and posterior cervical vertebrae of Spinophorosaurus (GCP-CV 4229) and Patagosaurus (MACN-CH 936). Unlike Bagualia, Spinophorosaurus also has lateral extensions at the end of the neural spine, which appear to be the distal ends of the spinodiapophyseal laminae (SPDLs).

Fourth cervical vertebra. The fourth cervical vertebra (MPEF-PV 3301/11) is lateromedially compressed and deformed, so that it tilts laterally at an angle of about $45^{\circ}$ to the base of its centrum (Figure 5). This deformation has caused the right zygapophyses to meet at unequal angles, and to make the ventral face of the centrum visible in right lateral view. This vertebra is more elongated than the previous vertebrae (El: 4.1; see Table 3 in Appendix 1).

As in most sauropods, both sides of the centrum have well-defined pleurocoels (Wilson and Sereno, 1998) that occupy almost its entire length (Figure 5A, C), similar to the condition of the more anterior cervical vertebrae described above. These pleurocoels are excavated more deeply in the anterior portion. Though the dorsal, ventral, and anterior margins of the pleurocoels are well marked, the posterior margin is absent, so the pleurocoel fades posteriorly as in Patagosaurus (Holwerda et al., 2021, figure 5) and Spinophorosaurus (Remes et al., 2009, figure 3A). The septum that separates the pleurocoels is extremely narrow and has a rough texture. The pleurocoels are not connected with any internal chambers or other pneumatic structures, as is common in most nonneosauropod sauropods with few exceptions, such as some mamenchisaurids with camellate internal cavities (e.g., Mamenchisaurus; Wedel, 2003, 


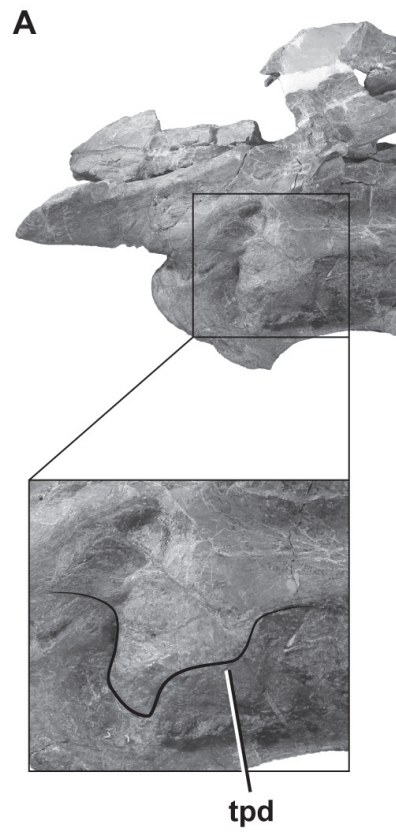

D

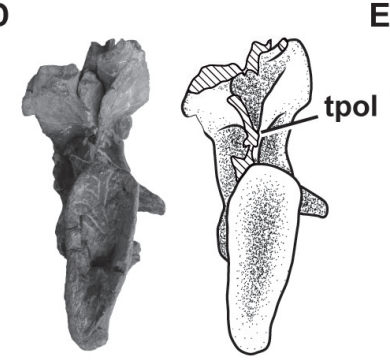

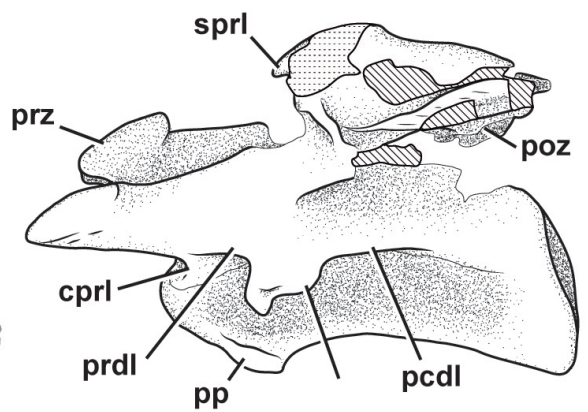

B

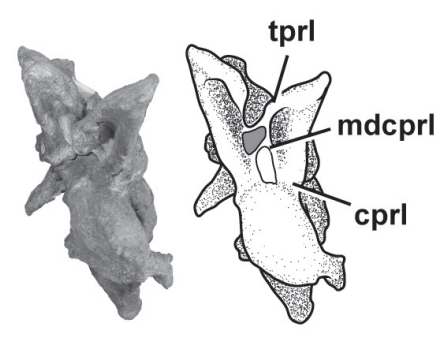

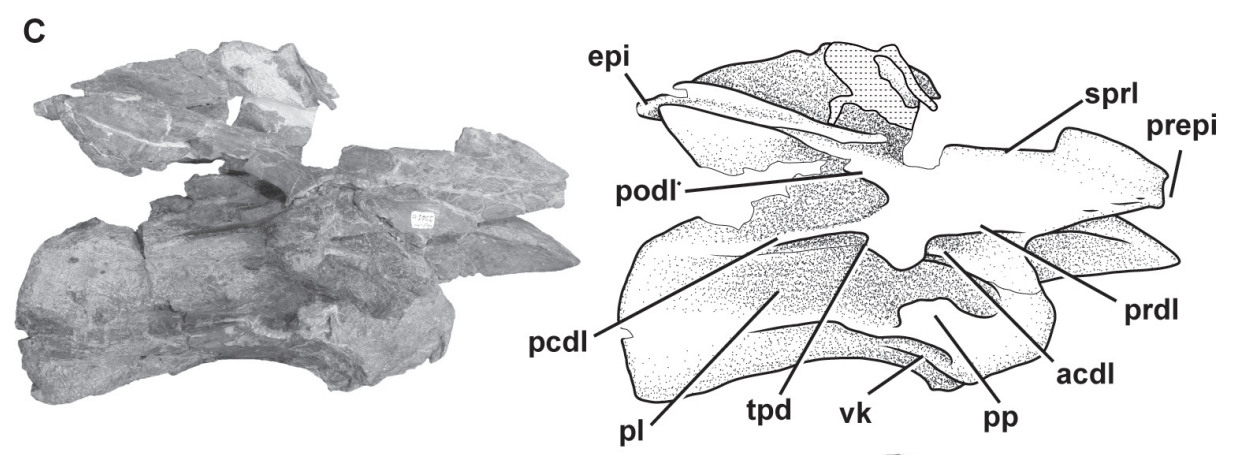

$\mathrm{E}$
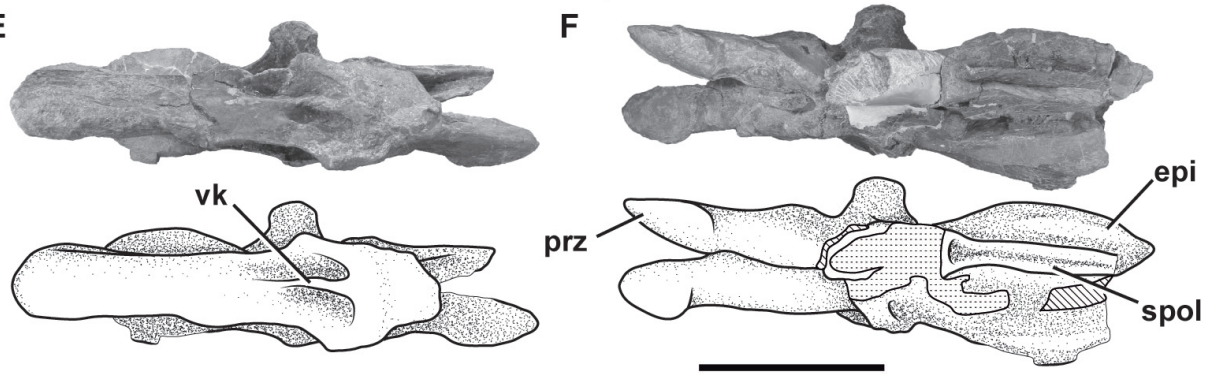

FIGURE 5. Fourth cervical vertebra of Bagualia alba (MPEF-PV 3301/11) in left lateral (A), anterior (B), right lateral $(\mathbf{C})$, posterior $(\mathbf{D})$, ventral $(\mathbf{E})$, and dorsal $(\mathbf{F})$ views. Abbreviations: acdl, anterior centrodiapophyseal lamina; $\mathbf{c p r l}$, centroprezygapophyseal lamina; dp, diapophysis; epi, epipophysis; mdcprl, medial division of the centroprezygapophyseal lamina; ns, neural spine; pcdl, posterior centrodiapophyseal lamina; pl, pleurocoel; podl, postzygodiapophyseal lamina; poz, postzygapophysis; pp, parapophysis; prdl, prezygodiapophyseal lamina; prepi, preepipophysis; prz, prezygapophysis; spol, spinopostzygapophyseal lamina; sprl, spinoprezygapophyseal lamina; tpd, triangular process of the diapophysis; tprl, intraprezygapophyseal lamina; vk, ventral keel. Hatched pattern represents broken surfaces and dotted pattern represents putty. Scale bar equals $10 \mathrm{~cm}$.

2005). The parapophyses are well developed, having an elliptic shape with the major axis oriented anteroposteriorly. They are situated at the anteroventral corner of the centrum. At the ventral surface of the centrum, there is a marked ventral keel that extends posteriorly for more than a third of the total length of the centrum (Figure 5E), as seen in other sauropods (e.g., Tazoudasaurus, Spinophorosaurus, Patagosaurus; Allain and Aquesbi, 2008; GCP-CV 4229; MACN-CH936). On each side of the ventral keel, there is a deep fossa which fades towards the posterior portion of the centrum, as in other sauropods (e.g., Amygdalodon, Tazoudasaurus, Jobaria; Rauhut, 2003, figure 1D; Allain and
Aquesbi, 2008, figure 9E-F; MNN TIG 3-5). The zipper-like neurocentral suture is also visible in this vertebra. The transverse processes are well-developed and directed lateroventrally. The development of diapophyseal laminae is greater than in the third cervical. The PCDL runs posteroventrally from the diapophysis to its contact with the centrum. On the posterior margin of the transverse process, there is a triangular process (Figure $5 \mathrm{~A}, \mathrm{C}$ ) that is also present in other sauropods (e.g., Shunosaurus, Cetiosaurus, Spinophorosaurus, Omeisaurus, Turiasaurus; ZDM T5401; LCM G468.1986; Remes et al., 2009; He et al., 1988, figures 20-21; CPT 1220). Patagosaurus does not have this trian- 
gular process in the holotype (PVL 4170), although it is found in a cervical vertebra referred to this taxon (MACN-CH 936). On both sides, the PCDL extend almost to the posterior articular face. This lamina traverses the vertebra with an inclination hardly greater than that observed in the previous cervical, resulting from the higher position of the diapophysis. The ACDLs are well marked and define, together with the PRDL, a deep PRCDF. Below the transverse process, there is a CDF delimited by the ACDL (anteriorly), the PCDL (posteriorly), and the dorsal edge of the pleurocoel (ventrally). Also, between the PCDL, and the PODL, a POCDF is developed. The PODL has a greater angle than that of the anteriormost cervical vertebrae described above. The prezygapophyseal process surpasses the anterior end of the centrum, and its articular surface is flat, whereas the lateral face is convex. The right prezygapophyseal process is directed dorsally at an angle of $30^{\circ}$ with respect to the left one, which is horizontal. As in the anterior vertebra, small horizontal striations can be recognized on the lateral surface of the prezygapophyseal process, and a rough protuberance on the dorsal surface of the diapophysis process (Figure $5 \mathrm{~A}$ ). In the distal ventral margin of the prezygapophyseal process, there is a protruding preepipophysis that reaches the anterior end of the process without exceeding it. Pre-epipophyses were initially described as a diagnostic character of Jobaria (Sereno et al., 1999; Wilson, 2002; Upchurch et al., 2004), but are also present in other non-neosauropod eusauropods (e.g., Shunosaurus, Spinophorosaurus, Patagosaurus, Mamenchisaurus, Turiasaurus; IVPP V 9065; GCP-CV-4229; PVL 4170, MACN CH 936; Ouyang and Ye, 2002, figure 17; CPT 1220) and some more derived taxa (e.g., Camarasaurus, Apatosaurus; BYU 9047, CM 84), so its distribution is greater than previously thought. In some taxa, the pre-epipophyses extend on the lateral side of the prezygapophyseal process towards the diapophysis as a tall, rugose crest (e.g., Klamelisaurus, Moabosaurus, Euhelopus, Kaatedocus; Moore et al., 2020; Britt et al., 2017, figure 16B; Wilson and Upchurch, 2009, figure 9; Tschopp et al., 2015, figure $51 \mathrm{~A}$ ) or as low striations, as seen in Bagualia (also, e.g., Turiasaurus, Spinophorosaurus, Patagosaurus, Malawisaurus; CPT 1220; GCP-CV4229 / HB1; MACN-CH 936; Gomani, 2005, figure 9). These lateral striations in Bagualia coincide with the site of attachment for epaxial musculature (Wedel and Sanders, 2002; Tsuihiji, 2005).
The CPRLs are short and define, together with the lateral walls of the neural canal and the TPRL, deep CPRFs (Figure 5B). The TPRLs contact each other medially, close to the roof of the neural canal. Only the proximal and distal (at the distal tip of the neural spine) portions of the thin SPRL are preserved. The right postzygapophysis is approximately $2 \mathrm{~cm}$ above the left one due to the deformation, and its articular surfaces are flat and sheet-like. Both postzygapophyses have an angle of approximately 30 degrees with respect to the horizontal plane of the centrum, which is common in sauropods, and causes them to always be located above the prezygapophyses and exceed the posterior face of the centrum (e.g., Shunosaurus, Spinophorosaurus; Zhang, 1988, figures 2427; Remes et al., 2009, figure 3A). In contrast, the basal sauropodomorphs have postzygapophyses with a low angle, so they are located at the same height or slightly higher than the prezygapophyses (e.g., Yizhousaurus, Adeopapposaurus, Leyesaurus; Zhang et al., 2018, figure 4A; Martínez, 2009, figure 15; Apaldetti et al., 2011, figure 7). There are no CPOLs. The TPOL is thin, and due to the lack of the portion between this lamina and the roof of the neural canal, it is not possible to say with certainty if there is a sTPOL (Figure 5D). Finally, the SPOLs are well defined but fuse at the posterior end of the neural spine.

The finger-shaped epipophyses extend posteriorly from the posterior margin of the postzygapophysis (Figure 5C, F). The right epipophysis exceeds (by about $0.5 \mathrm{~cm}$ ) the posterior margin of the postzygapophysis, whereas this extension is not preserved on the other side. On the right lateral side there is preserved a proximal portion of the EPRL (Figure 5C). This lamina is better developed in the mid and posterior cervicals (see below). On the left side there is a vertical lamina extending from the PODL to the anterior margin of the neural spine, although this may be due to asymmetry in the vertebra.

Despite the neural spine being poorly preserved, it is clearly low, longer anteroposteriorly than medially, and is about $30 \%$ of the length of the centrum. It is around three times longer than tall. Its width is constant along the anteroposterior length, whereas this increases to around four times more in the dorsal portion than its base. The dorsoventral height of the neural spine is equal to half of the height of the vertebral centrum.

Fifth cervical vertebra. This vertebra (MPEF-PV 3301/14) is well preserved but was strongly deformed by the force of lateromedial compression 


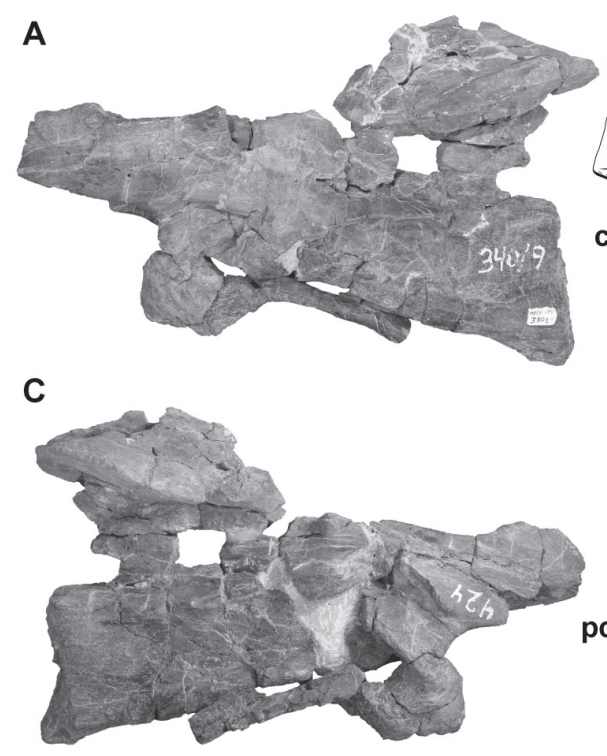

E
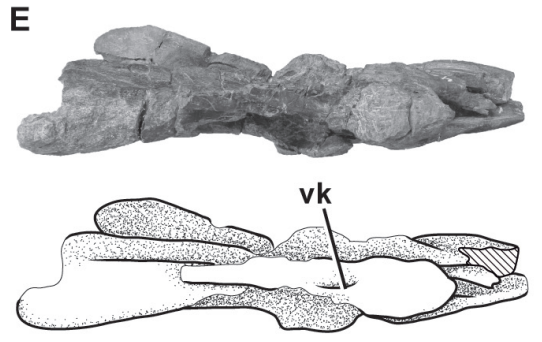
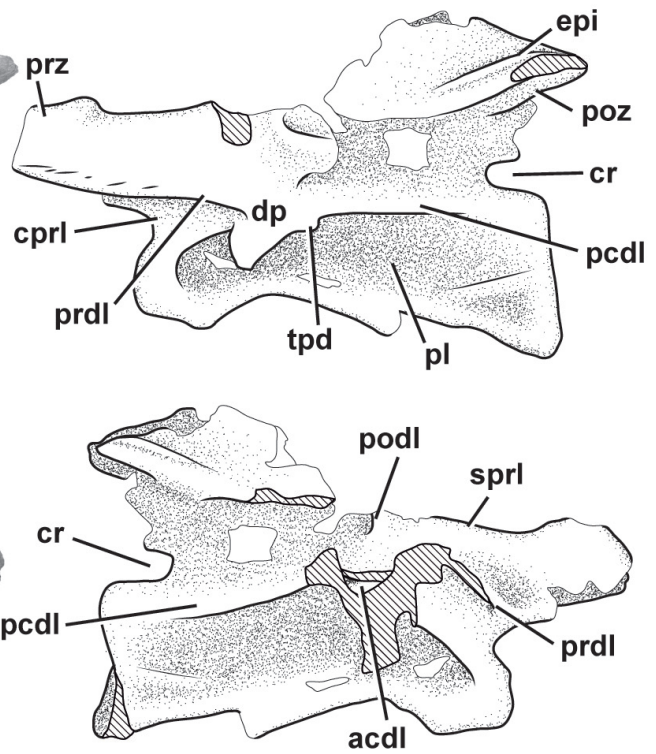

D

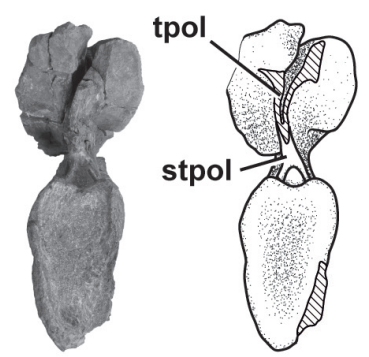

$\mathbf{F}$
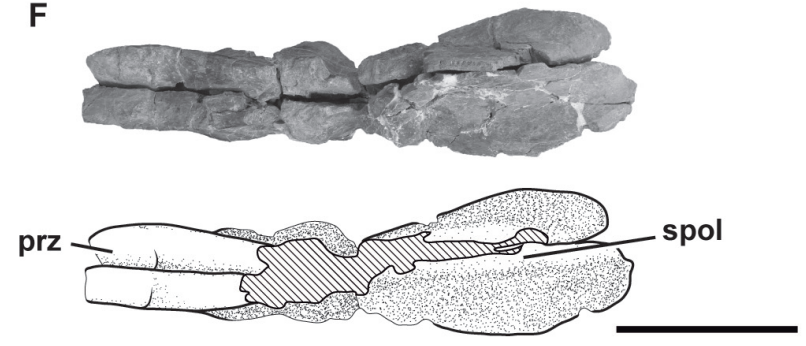

FIGURE 6. Fifth cervical vertebra of Bagualia alba (MPEF-PV 3301/14) in left lateral (A), anterior (B), right lateral (C), posterior (D), ventral (E), and dorsal (F) views. Abbreviations: acdl, anterior centrodiapophyseal lamina; cprl, centroprezygapophyseal lamina; cr, caudal recess; dp, diapophysis; epi, epipophysis; pcdl, posterior centrodiapophyseal lamina; pl, pleurocoel; podl, postzygodiapophyseal lamina; poz, postzygapophysis; prdI, prezygodiapophyseal lamina; prz, prezygapophysis; sprl, spinoprezygapophyseal lamina; spol, spinopostzygapophyseal lamina; stpol, single intrapostzygapophyseal lamina; tpd, triangular process of the diapophysis; tpol, intrapostzygapophyseal lamina; tprl, intraprezygapophyseal lamina; vk, ventral keel. Hatched pattern represents broken surfaces. Scale bar equals $10 \mathrm{~cm}$.

during the diagenetic process (Figure 6). As a result of this deformation, the posterior portion of the centrum, the neural spine, and the postzygapophyses are slightly inclined to the right. This vertebra has an elongate centrum with an El of 3.8 (see Table 3 in the Appendix 1). The ventral surface of the centrum bears a short keel (Figure 6E), which is restricted to the anterior portion of the centrum, being relatively shorter than that of more anterior cervical vertebrae. This shortening of the keel on a middle cervical vertebra also occurs in Spinophorosaurus (GCP-CV-4229), but it does not happen in Patagosaurus, where the ventral keel exceeds half the length of the centrum (MACN-CH 936; Holwerda et al., 2021, figure 6E). The wellmarked pleurocoel occupies almost the entire length of the centrum (Figure 6A, C). The septum that separates left and right pleurocoels is wider than that of anterior cervical vertebrae, although it maintains the rough texture described above. As in more anterior cervicals, the pleurocoels are not communicating with internal pneumatic cavities. On the posteroventral corner of the centrum there is a triangular deepening, separated from the pleurocoel by a low and thin crest that runs posterodorsally from the ventral margin of the centrum to reach the posterior articular surface (Figure 6A, C). The parapophyses are low and slightly ventrally projected, and the cervical ribs were not fused.

The maximum height of the neural arch is similar to the maximum height of the posterior articular surface of the centrum. As in more anterior cervical vertebrae, the base of the neural arch is triangular in anterior and posterior views, and the neural canal is semicircular (higher than wide), which seems to be a preservational artifact due to lateromedial compression. In this element, the neurocentral suture is still visible anteriorly, but is less 
distinct than in the more anterior cervical vertebrae. This is interpreted as evidence of greater fusion with the centrum than in the anterior vertebrae and a neurocentral closure pattern from posterior to anterior in both the cervical series as a whole and within each vertebra, a typical feature of sauropods (Irmis, 2007). As in the third cervical vertebra, there is a caudal recess in the posteroventral end of the neural arch, although it is larger. Only the left transverse process is preserved. The transverse process is lateroventrally oriented, but at a lower angle than those of more anterior cervical vertebrae. The ACDL is a short and vertical lamina positioned just below the transverse process as is seen in Spinophorosaurus (GCP-CV 4229).

The PCDL extends from the posterior margin of the transverse process to close to the posterior articular surface of the centrum. As in the fourth cervical vertebra, there is a triangular process on the posterior margin of the transverse process. The prezygapophyses are directed anteriorly and horizontally, surpassing the anterior articular face of the centrum by $7 \mathrm{~cm}$. The articular surface is slightly convex and faces dorsomedially at an angle of around 45 degrees. The CPRLs are stout and single laminae. A thin TPRL runs medially to both prezygapophyses (Figure 6B), and it is not possible to recognize a STPRL due to poor preservation of this zone. The PRDL is preserved on the left side of this vertebra and is well developed, extending posteroventrally from the ventral margin of the prezygapophysis at a lower angle than the fourth cervical vertebra. As previously described, on the lateral surface of the prezygapophyseal process, close to the anterior margin of the PRDL, there are numerous, small, horizontal striations. The PODLs are directed dorsally at an angle of 30 degrees. From the left PODL, a smooth EPRL appears to extend towards the SPRL, although this area is badly damaged. The postzygapophyses are flat, and the dorsolateral surfaces bear weak epipophyses that extend posteriorly but do not pass beyond the posterior margin of the postzygapophyses (Figure 6A). Therefore, the epipophyses of this vertebra are not as posteriorly developed as those of the more anterior cervical vertebrae, but are dorsoventrally higher. Both postzygapophyses are medially connected to each other through a thin TPOL. The STPOL runs from the roof of the neural canal to the TPOL. The STPOL is longer than the present in the third cervical vertebra (Figure 6D). The elongation of the STPOL through the series is common in other sauropods (e.g., Cetiosaurus,
Camarasaurus, Apatosaurus; Carballido and Sander, 2014).

The neural spine is poorly preserved, missing most of their anterior half and its dorsal margin. The thin SPOLs are close to each other due to the lateromedial compression of this element (Figure $6 \mathrm{~F})$.

Sixth cervical vertebra. The sixth cervical vertebra (MPEF-PV 3301/15) is incomplete, lacking the anterior half of its centrum, so the parapophyses and the ventral keel (if was present) were not preserved (Figure 7). As in the other, more anterior cervical vertebrae, it is lateromedially compressed. This deformation also caused the right zygapophyses to be $3 \mathrm{~cm}$ below the level of the left ones. Both sides of the centrum have well-marked pleurocoels. The preserved posterior half of the pleurocoel extends to near the posterior margin of the centrum (Figure 7A, C). As in the fifth cervical vertebra, on the posteroventral corner of the centrum there is a triangular deepening, which is separated anterodorsally from the pleurocoel by a thin, almost horizontal lamina. This deepening is shallower than in the fifth cervical vertebra. This feature is also seen in Amygdalodon (MLP 46-VIII-21-1/8) and Spinophorosaurus (GCP-CV 4229), but is absent in most other sauropods (e.g., Cetiosaurus, Haplocantosaurus, Camarasaurus, Giraffatitan). The septum separating the pleurocoels is thicker than that of the fifth cervical vertebra, and exhibits the rough lateral texture also observed in the other elements of the holotype.

The neurocentral suture, recognized in the previous vertebrae, is not visible in this element, but its presence is somewhat uncertain considering that its anterior portion is not preserved. As in the third and fifth cervical vertebrae, there is a caudal recess in the base of the neural arch. The transverse processes are directed lateroventrally, with the diapophyses facing ventrally. The transverse process is ventrally supported by a short, vertical ACDL, slightly more developed in this vertebra than in the more anterior one. The PCDL extends from the diapophysis to near the dorsal margin of the posterior face of the centrum. This lamina is longer than those present in the middle cervical vertebrae of other sauropods, such as Volkheimeria (PVL4077), Spinophorosaurus (GCP-CV 4229), and Patagosaurus (PLV 4170).

The prezygapophyseal processes are horizontally oriented and anteriorly directed, with sheet-like flat surfaces facing dorsolaterally at an angle between 75 degrees (the left one) and 45 degrees (the right one). As in the fourth cervical 
A
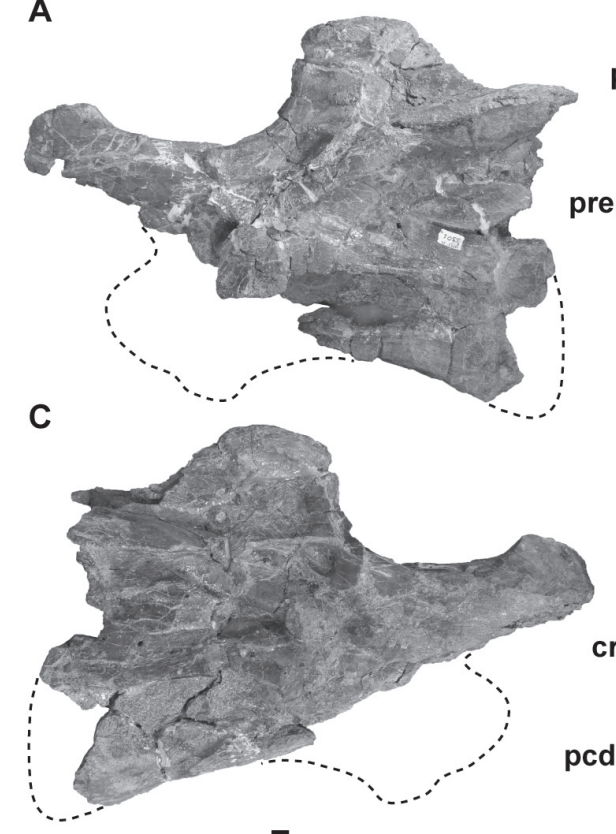

E

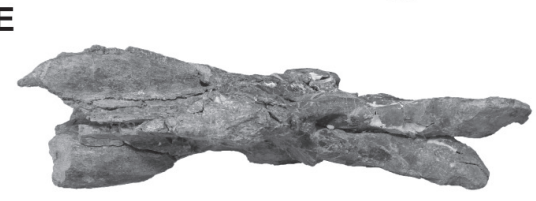

B

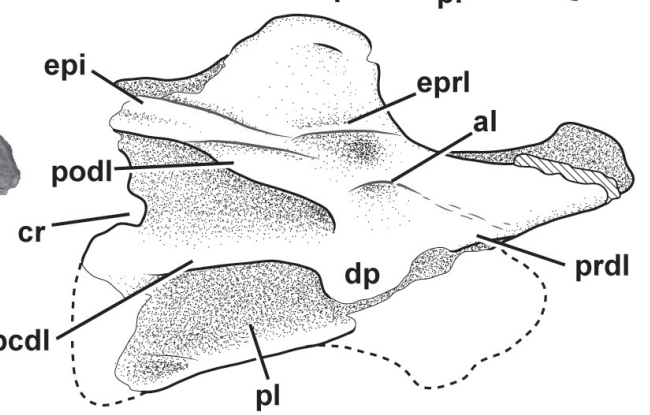

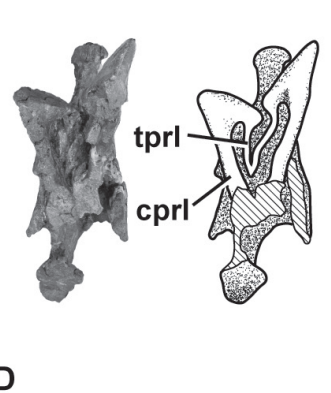
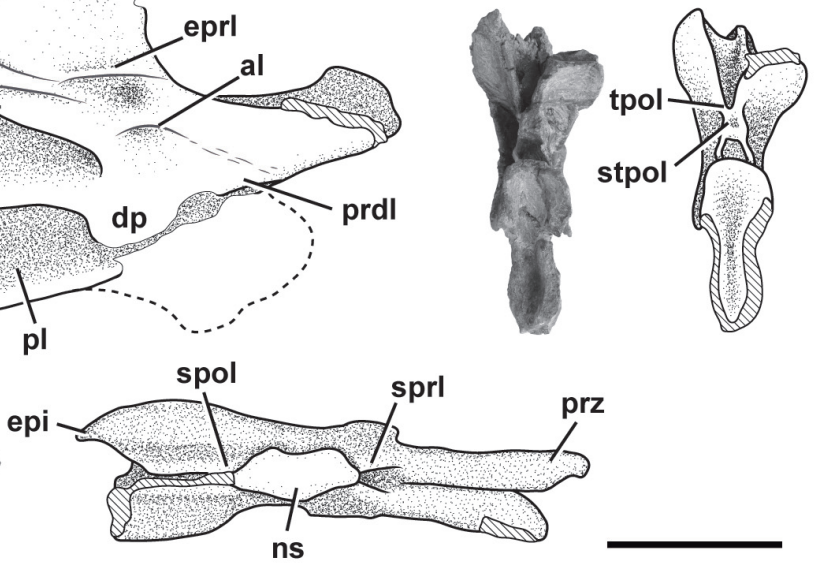

FIGURE 7. Sixth cervical vertebra of Bagualia alba (MPEF-PV 3301/15) in left lateral (A), anterior (B), right lateral (C), posterior (D), and dorsal (E) views. Abbreviations: al, accessory lamina; cprl, centroprezygapophyseal lamina; $\mathbf{c r}$, caudal recess; dp, diapophysis; epi, epipophysis; eprl, epipophyseal-prezygapophyseal lamina; ns, neural spine; pcdl, posterior centrodiapophyseal lamina; pl, pleurocoel; podl, postzygodiapophyseal lamina; poz, postzygapophysis; prdl, prezygodiapophyseal lamina; prepi, pre-epipophysis; prz, prezygapophysis; spol, spinopostzygapophyseal lamina; sprl, spinoprezygapophyseal lamina; stpol, single intrapostzygapophyseal lamina; tpd, triangular process of the diapophysis; tpol, intrapostzygapophyseal lamina; tprl, intraprezygapophyseal lamina. Dashed line for the reconstructed parts, and hatched pattern for broken surfaces. Scale bar equals $10 \mathrm{~cm}$.

vertebra, there is a pre-epipophysis on the distal ventral margin of the prezygapophyseal process. In this case, the pre-epipophysis is better developed than that of the fourth cervical vertebra, forming an anteroventrally directed protrusion that extends slightly past the anterior end of the prezygapophyses. The lateral surface of the prezygapophyseal process has numerous horizontal striations continuous with the pre-epipophysis. The PRDL are poorly preserved on both sides. The TPRL is narrow and long (Figure 7B). Due to poor preservation of the anteroventral portion of the neural arch, it is not possible to recognize the presence of a STPRL.

The ACDL and PCDL enclose a small CDF, which lies just below the transverse process. As in the anterior vertebrae there is a triangular process on the posterior margin of the transverse process. The PODL is posterodorsally directed from the diapophysis at an angle of 50 degrees, to contact the postzygapophysis anteriorly. This lamina is straight in dorsal view, as in most sauropods (e.g., Tazoudasaurus, Cetiosaurus, Jobaria, Nigersaurus; Allain and Aquesbi, 2008, figure 9; Upchurch and Martin, 2002, figure 2; MNN TIG 3-5; MNN GAD 512). However, the PODL is concave in dorsal view in middle cervical vertebrae of certain taxa (e.g., Spinophorosaurus, Patagosaurus, Mamenchisaurus, Euhelopus; GCP-CV 4229; MACN-CH 936; Ouyang and Ye, 2002, figure 15; Wilson and Upchurch, 2009, figure 11). The PODL delimits a deep POCDF dorsally. As described in the fourth cervical vertebra, an EPRL projects anteriorly from the lateral margin of the postzygapophysis onto the SDF, converging with the SPRL (Figure 7A, C). The EPRL was initially identified as a synapomorphic feature of Rebbachisauridae (e.g., Nigersaurus, Demandasaurus, Zapalasaurus; Sereno et al., 2007; Torcida et al., 2011; Salgado et al., 2006), but this lamina presents a broader distribution, and has also been recognized in some basal macronar- 
ians, such as Camarasaurus (BYU 9047) and Euhelopus (Wilson and Upchurch, 2009, figures 910 ), and the titanosaur Rapetosaurus (Curry Rogers, 2009). Within non-neosauropod sauropodomorphs, a similar lamina to that seen in Bagualia is present in a few basal eusauropods (e.g., Moabosaurus, Klamelisaurus; Britt et al., 2017, figures 13G, 14B, 16B, 17B; Moore et al., 2020). In contrast to Bagualia and the aforementioned taxa, the posterior cervical vertebrae of Patagosaurus exhibit a short, smooth lamina restricted within the SDF and without contacting any other structure (MACN-CH 936), so this lamina is not interpreted here as a true EPRL. A second lamina within the SDF is present below the EPRL in Bagualia, which is less marked and more curved. Both laminae are smooth and define small subfossae within the SDF (Figure 7A, C). The postzygapophyses are flat and visible laterally. There are no CPOLs developed, as the lateral walls of the neural canal are thin and dorsally connected to the sTPOL. The STPOL forms the roof of the neural canal and connects it to the ventral margin of the TPOLs (Figure 7D). The TPOLs are ventromedially directed from the postzygapophyses at an angle of some 80 degrees. The complete left epipophysis extends posteriorly around $0.5 \mathrm{~cm}$ from the posterior end of the postzygapophysis.

The neural spine is rectangular, with rounded anterior and posterior edges in lateral view. Its anteroposterior length is three times its lateromedial width. The dorsoventral height is less than the dorsoventral height of the vertebral centrum (measured on its posterior articular surface). The SPRLs are well developed and are joined in the mid dorsoventral length of the neural spine (Figure 7E). In this vertebra it seems that the SPRLs are not anteriorly extended as in cervical 3 . The SPOL is thin and reaches the posterior dorsal end of the neural spine.

Seventh cervical vertebra. MPEF-PV 3301/16 has been deformed by lateromedial compression, which caused the right side to be above the left one (Figure 8). The vertebra is almost complete, lacking only its left postzygapophysis and a fragment of its middle ventral portion of the centrum. This centrum has the greatest elongation index in the preserved cervical series of the holotype specimen MPEF-PV 3301 (aEl: 4.6; see Table 3 in the Appendix 1). The ventral surface of the centrum has two well-marked medial keels, an anterior and a posterior one (Figure $8 \mathrm{~F}$ ). The anterior one is 4.5 $\mathrm{cm}$ long and is slightly longer than the posterior one, and is positioned at the level of the parapoph- yses and is bounded by two shallow fossae. The posterior ventral keel extends around $3 \mathrm{~cm}$ anterior to the posterior articular surface of the centrum. This accessory posterior keel is also seen in other mid-posterior cervical vertebrae from the referred specimens (see below). This posterior keel is also present in Spinophorosaurus (GCP-CV 4229), but absent in Patagosaurus (MACN-CH 936; Holwerda et al., 2021, figure $8 \mathrm{C}$ ). Both sides of the centrum have well-marked pleurocoels that occupy almost the entire anteroposterior length of the centrum (Figure 8A, D). The septum that separates the pleurocoels is thicker than in the sixth cervical vertebra, following the trend of posterior widening, but has a similar rough texture. As in the anterior vertebrae, there is a deep, triangular fossa on the posteroventral corner of the centrum, although in this vertebra it is shallower, and the anterodorsal crest is low. The right parapophysis is almost complete and lateroventrally directed, whereas the left one is shorter, rounded, and dorsolaterally directed as a result of taphonomic deformation. As in more anterior cervical vertebrae, the cervical ribs were not fused to the centrum.

The base of the neural arch is triangular in shape in anterior view, but the neural canal is ovoid and compressed lateromedially. A zipper-like neurocentral suture is weakly marked at the anterior end of this element. The right transverse process and its diapophysis are complete whereas the left transverse process is broken, only preserving some of the laminae that connect the diapophysis to the centrum and neural arch. The ACDL is a short and slightly anteroventrally oriented lamina positioned just below the transverse process. The PCDL is longer than the ACDL and extends from the posterior margin of the transverse process to the posterodorsal surface of the centrum. As in the previous vertebrae, there is a triangular process on the posterior margin of the transverse process, which is present in other mid-cervical vertebrae found in the quarry (see below). Below the transverse process there is a deep, triangular CDF defined anterodorsally by the ACDL, posterodorsally by the PCDL, and ventrally by the centrum. Posteriorly and parallel to the ACDL and below the $\mathrm{PCDL}$, there is a short accessory lamina (Figure $8 \mathrm{~A}$ ). This is an uncommon lamina, which has previously been described in the rebbachisaurid Nigersaurus (MNN GAD 512) and the macronarian Europasaurus, and in the latter taxon was called the accessory ACDL (Carballido and Sander, 2014, figures $4 \mathrm{D}, 7 \mathrm{E})$. 

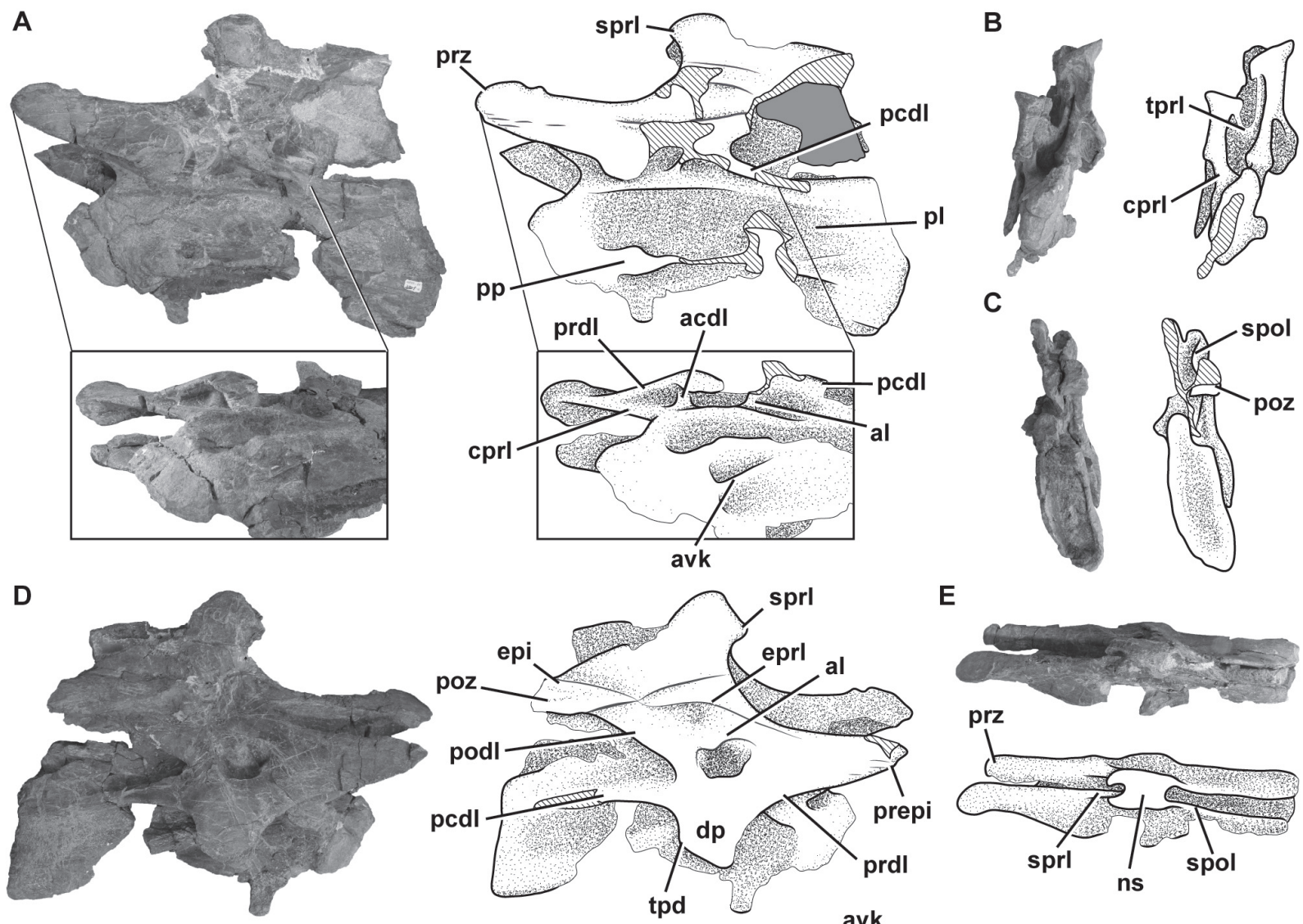

E
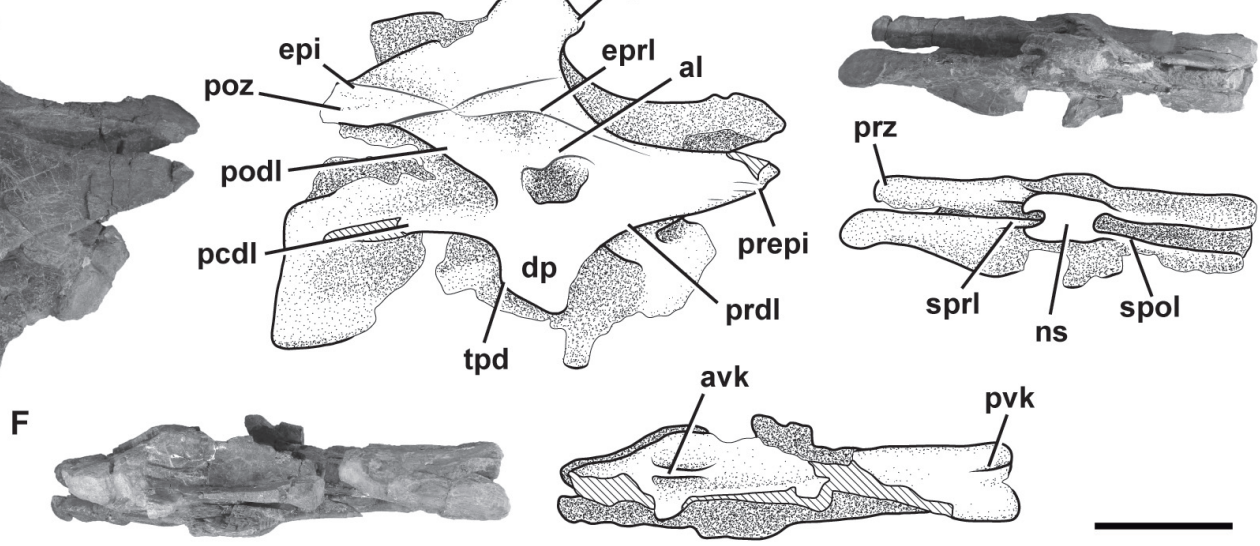

FIGURE 8. Seventh cervical vertebra of Bagualia alba (MPEF-PV 3301/16) in left lateral and ventrolateral (A), anterior (B), posterior (C), right lateral (D), dorsal (E), and ventral (F) views. Abbreviations: acdl, anterior centrodiapophyseal lamina; al, accessory lamina; avk, anterior ventral keel; cprl, centroprezygapophyseal lamina; dp, diapophysis; epi, epipophysis; eprl, epipophyseal-prezygapophyseal lamina; ns, neural spine; pcdl, posterior centrodiapophyseal lamina; pl, pleurocoel; podl, postzygodiapophyseal lamina; poz, postzygapophysis; pp, parapophysis; prdl, prezygodiapophyseal lamina; prepi, pre-epipophysis; prz, prezygapophysis; pvk, posterior ventral keel; spol, spinopostzygapophyseal lamina; sprl, spinoprezygapophyseal lamina; tpd, triangular process of the diapophysis; tprl, intraprezygapophyseal lamina. Hatched pattern represents broken surfaces. Scale bar equals $10 \mathrm{~cm}$.

The CPRL is long and forms the lateral walls of the neural canal, while a thin and short TPRL (poorly preserved) is directed ventrally, directly contacting the roof of the neural canal (Figure 8B). The prezygapophyses have flat, sheet-like articular surfaces. Both are directed anteriorly, parallel to the plane of the centrum, and extend past the condyle for about $4 \mathrm{~cm}$. As in the fourth and sixth cervical vertebrae, there is a marked pre-epipophysis, although it is more weakly developed and does not reach the anterior tip of the prezygapophysis. The lateral surface of the prezygapophyseal process possesses small horizontal striations, as have been described for the anterior vertebrae. The PRDL also has a greater angle of inclination than in the anterior vertebrae. The right postzygapophysis is complete and well preserved, whereas the left one is broken. The preserved postzygapophysis is directed horizontally backward without extending beyond the posterior surface of the centrum. Its lateral edge is convex and the epipophysis is present and prominent, being stout and dorsoventrally high. Whereas the epipophyses of the anteriormost cervical vertebrae are posteriorly directed, surpassing the postzygapophysis, in this vertebra the posterior edge of the epipophysis is at 
the same level as the postzygapophysis. Below the postzygapophyses, it is not possible to recognize the TPOLs or STPOL due to lateral crushing of the vertebra and general poor preservation. As has been described in the anterior vertebrae, there are EPRLs (Figure 8A, D). This lamina is long and defines the dorsal margin of a small fossa within the SDF. Beneath this small fossa, there is an accessory lamina that does not contact any other lamina and that defines a very deep fossa on the dorsal surface of the transverse process, although this is likely to be due to the crushing of the vertebra.

The neural spine is oriented slightly anteriorly and is anteroposteriorly short, being around one sixth the length of the centrum. The distal end is around twice as lateromedially wide than the base of the spine, and is 1.5 times anteroposteriorly longer than lateromedially wide at its distal tip. The dorsoventral length of the spine is less than the dorsoventral length of the vertebral centrum. The SPRL extends from the dorsal surface of the prezygapophysis to the distal end of the neural spine (Figure 8E). As in the third cervical vertebra, both SPRLs extend anteriorly at the distal end of the spine. The SPOLs are thin and extend from the postzygapophyses to the posterodorsal margin of the neural spine (Figure $8 \mathrm{C}$ ). Both anteriorly and posteriorly, deep fossae are enclosed between the SPRLs and SPOLs, respectively. Neither PRSL nor POSL are present and the anterior and posterior surfaces are rough. As in Patagosaurus (Holwerda et al., 2021), the SPDL is not present in any cervical vertebrae of Bagualia.

MPEF-PV 11040. This specimen consists of two middle cervical vertebrae (which, by comparison to the holotype specimen, are interpreted as the fourth and fifth or perhaps the fifth and sixth cervical vertebrae). As this specimen overlaps with two of the cervical vertebrae previously described (either 4-5 or 5-6), only a brief description is given here.

The more anterior vertebra (MPEF-PV 11040/ 1 ) is an incomplete neural arch (including the neural spine) that lacks its anterior portion, including the prezygapophyses and transverse processes (Figure 9). Both postzygapophyses are preserved and the thin, paired TPOL runs ventrally from them to contact the roof of the neural canal (Figure 9C). Above the postzygapophyses, there are weakly developed epipophyses, and although their posterior tips are missing, they project posteriorly to the postzygapophyses (as described for MPEF-PV 3301-13/11/15; Figure 9A, D, E). In this vertebra, an EPRL is preserved within the SDF, which is smooth and extends from the PODL to the SPRL and appears to have a slightly marked contact with the epipophysis (Figure 9A, D).

The neural spines of the fourth and fifth cervical vertebrae of the holotype were not preserved, making it impossible to directly compare the morphology of this specimen with the holotype. The neural spine is around four times anteroposteriorly longer than lateromedially wide. Based on the shortening trend described for the neural spine, and the proportions of the third and sixth vertebrae, this neural spine can be interpreted as a fourth or fifth position. The anterodorsal edge and the posterior portion of the neural spine are incomplete. Therefore, it is not possible to distinguish the presence of an anterior extension of the SPRLs (Figure 9B), which was present in the third, fourth, and seventh cervical vertebrae of MPEF-PV 3301. A thin SPOL runs from the postzygapophyses up to the posterodorsal end of the neural spine (Figure $9 \mathrm{E})$. The neural spine widens lateromedially towards its distal end (around twice that of its base). The dorsal surface is convex and rough with small striations anteroposteriorly oriented.

The following cervical vertebra of this specimen (MPEF-PV 11040/2) is nearly complete (Figure 10). The centrum is opistocoelus and elongated. The elongation index estimated for this element (aEl: 3.1 ; see Table 3 in Appendix 1) is not exact, given that the centrum is incomplete. The ventral surface is flat in transverse section, and bears two median keels, one at the anterior section, at the level of the parapophysis, and the other at the posterior third as seen in the seventh cervical vertebra of MPEF-PV 3301 (Figure 10F). Both keels have the same length (around $7 \mathrm{~cm}$ ) and extend towards the mid length of the centrum before fading. On each side of the anterior keel, there is a deep fossa that becomes deeper anteriorly. On the left side (where it is possible to observe), there is a concavity posterior to the parapophysis, which is almost continuous with the pleurocoel (Figure 10D). Dorsally-excavated parapophyses without a septum dividing them from the pleurocoels are also observed in Spinophorosaurus (GCP-CV 4229) and Patagosaurus (MACN$\mathrm{CH}$ 936, PVL 4170). In contrast, a marked septum is present in neosauropods (Upchurch, 1998). Both transverse processes are incomplete, but the right retains its posterior margin, where a portion of the triangular process described in MPEF-PV 3301 is present (Figure 10A). The ACDL is situated anterior to and below the transverse process and is ori- 

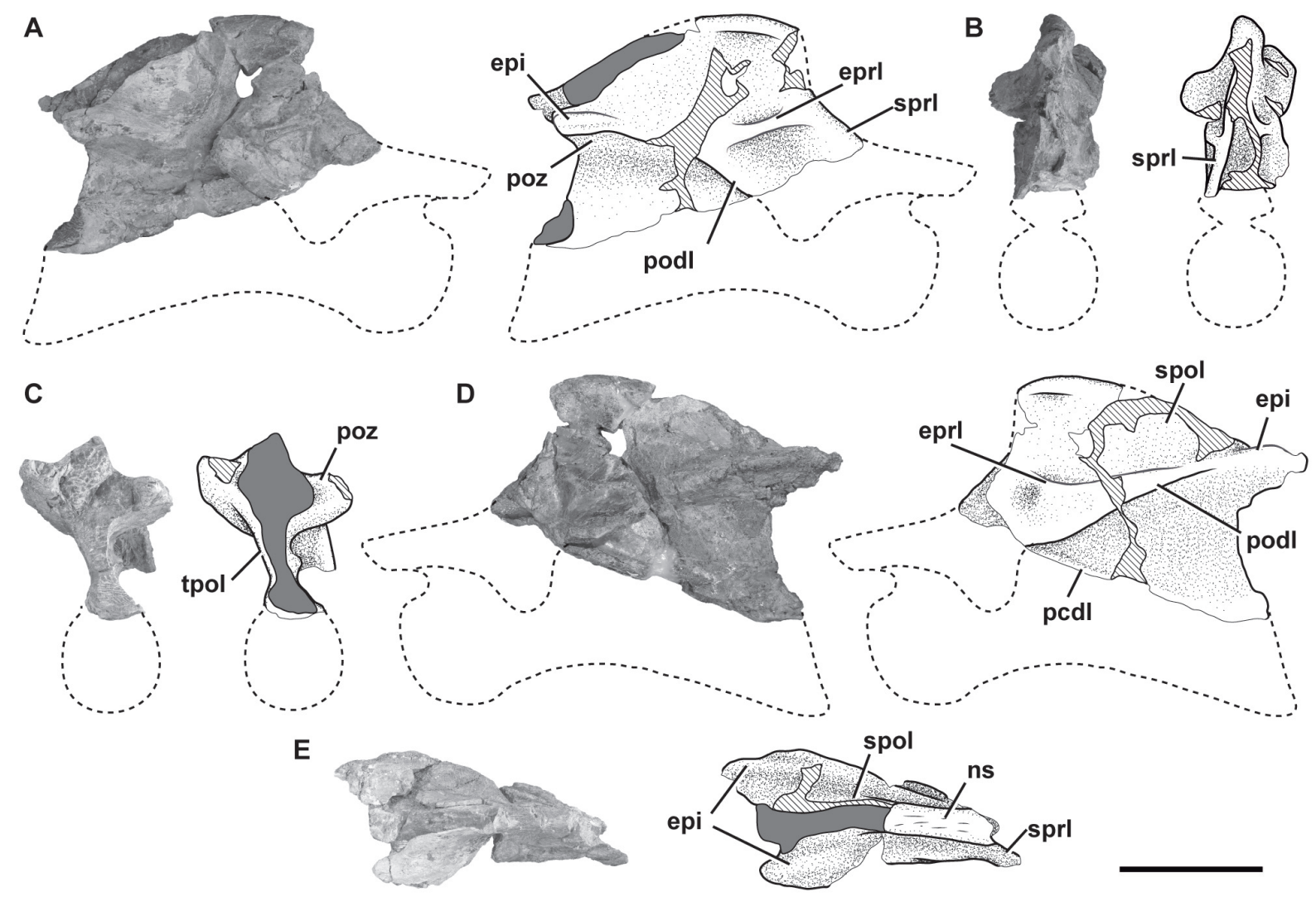

FIGURE 9. Middle cervical vertebra of Bagualia alba (MPEF-PV 11040/1) in right lateral (A), anterior (B, posterior (C), left lateral (D), and dorsal (E) views. Abbreviations: epi, epipophysis; eprl, epipophyseal-prezygapophyseal lamina; ns, neural spine; pcdl, posterior centrodiapophyseal lamina; podl, postzygodiapophyseal lamina; poz, postzygapophysis; sprl, spinoprezygapophyseal lamina; tpol, intrapostzygapophyseal lamina. Dashed line for the reconstructed parts. Hatched pattern represents broken surfaces and grey form represents sediment. Scale bar equals $10 \mathrm{~cm}$.

ented anteroventrally, contacting the CPRL before reaching the centrum. As in the seventh cervical vertebra, on the left side (where it is preserved) there is a short accessory lamina below the PCDL that runs anteroventrally (Figure 10D). Only the base of the left prezygapophyseal process is preserved. As seen in MPEF-PV 3301, the CPRL bifurcates in an mdCPRL that connects the centrum with the sTPRL (Figure 10B). The mdCPRL forms the walls of the neural canal and is present in most sauropods (e.g., Spinophorosaurus, Europasaurus; GCP-CV 4229; Carballido and Sander, 2014). The roof of the neural canal is connected with the TPRL via a thin STPRL. Both the CPRLs and TPRL delimit a single deep CPRF below the prezygapophyseal process on each side of the neural canal. This fossa is deeper than those present in the anterior cervical vertebrae (which preserve this area) of MPEF-PV 3301. This difference is interpreted as a product of the more posterior position of this cervical element. As described in the anterior vertebrae, there is an EPRL within the SDF which extends from the lateral margin of the postzygapophysis to the SPRL (Figure 10A, D). This lamina forms the dorsal margin of a deep subfossa within the SDF. Furthermore, there is an accessory lamina below the EPRL that does not contact any other lamina and defines a marked subfossa both dorsally and ventrally.

From the medial edge of the postzygapophyses the TPOLs run ventromedially to the roof of the neural canal (Figure 10C). The epipophyses are poorly developed on the dorsal surface of the postzygapophyses. The preserved portion of the left epipophysis extends posteriorly from the postzygapophysis, slightly surpassing the posterior articular surface of the centrum. From the dorsomedial surface of the postzygapophysis, the SPOL is oriented anterodorsally at an angle of 45 degrees towards the posterior margin of the distal tip of the neural spine. The SPOF is as deep as the SPRF and also occupied the total length of the neural spine. 
A

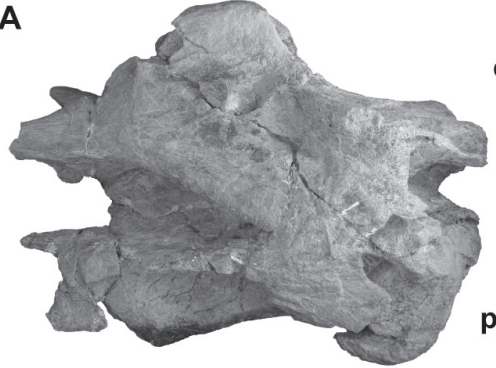

C

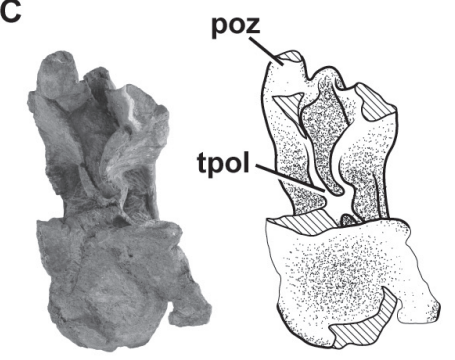

E
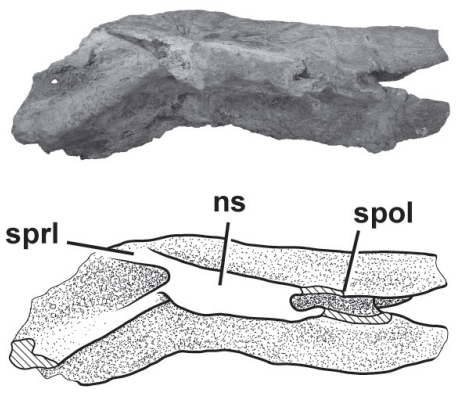
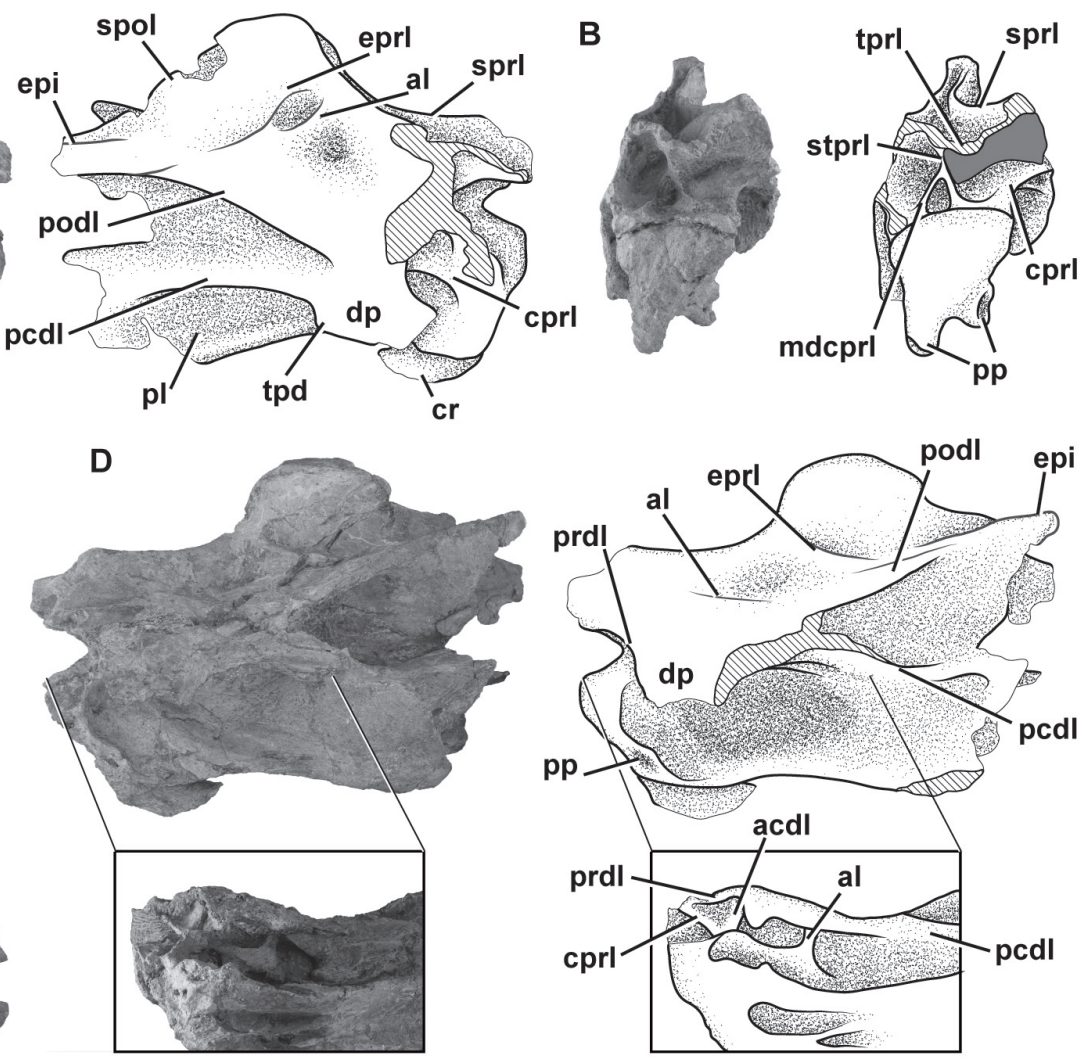

$\mathbf{F}$

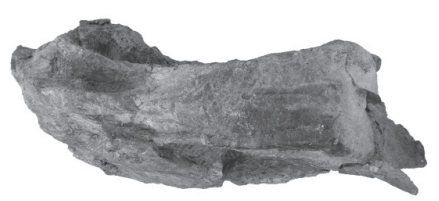

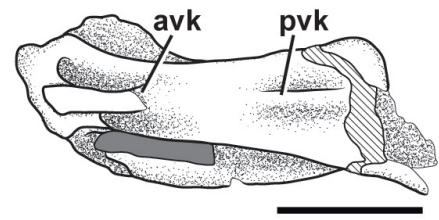

FIGURE 10. Middle cervical vertebra of Bagualia alba (MPEF-PV 11040/2) in right lateral (A), anterior (B), posterior (C), left lateral and lateroventral (D), dorsal (E), and ventral (F) views. Abbreviations: acdl, anterior centrodiapophyseal lamina; al, accessory lamina; avk, anterior ventral keel; cprl, centroprezygapophyseal lamina; cr, cervical rib; dp, diapophysis; epi, epipophysis; eprl, epipophyseal-prezygapophyseal lamina; mdcprl, medial division of the centroprezygapophyseal lamina; ns, neural spine; pcdl, posterior centrodiapophyseal lamina; pl, pleurocoel; podl, postzygodiapophyseal lamina; poz, postzygapophysis; pp, parapophysis; prdl, prezygodiapophyseal lamina; pvk, posterior ventral keel; spol, spinopostzygapophyseal lamina; sprl, spinoprezygapophyseal lamina; stprl, single intraprezygapophyseal lamina; tpol, intrapostzygapophyseal lamina; tpd, triangular process of the diapophysis; tprl, intraprezygapophyseal lamina. Hatched pattern represents broken surfaces and grey form represents sediment. Scale bar equals $10 \mathrm{~cm}$.

The neural spine widens lateromedially towards its distal end, being 2.7 times anteroposteriorly longer than lateromedially wide (like the sixth cervical of the holotype) and three times shorter than the total anteroposterior length of the centrum (Figure 10E). As the sixth cervical vertebra, the SPRLs do not extend anteriorly from the distal end of the neural spine.

MPEF-PV 3408. This cervical vertebra is almost complete, although strongly dorsoventrally crushed (Figure 11). Given its low elongation index and general morphology it is considered a posterior cervical vertebra. The centrum is opisthocoelous and has an El of 2.5 (see Table 3 in Appendix 1) with a long ventral keel (Figure 11E). The height of the keel decreases posteriorly from near the midlength of the centrum. At the anterior portion of the centrum, at the level of the parapophyses, the ventral keel is bounded by paired fossae, as was described for the middle cervical vertebrae (see above). In transverse section, the ventral surface is flat throughout the posterior half. The parapophy- 
A
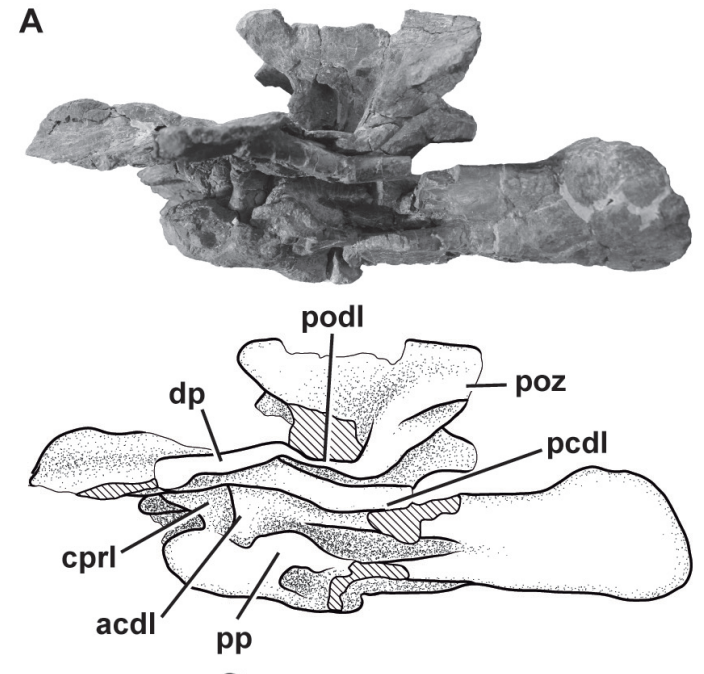

E

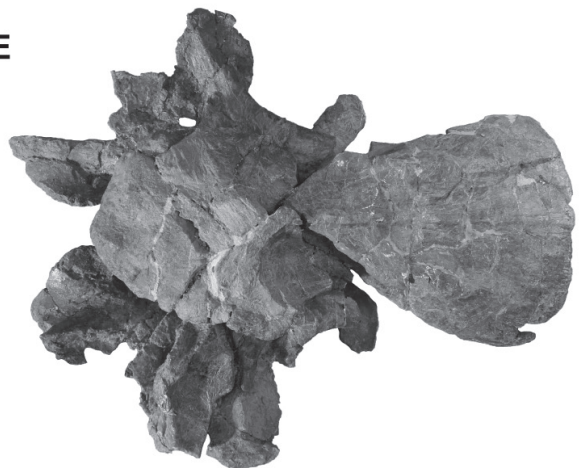

B

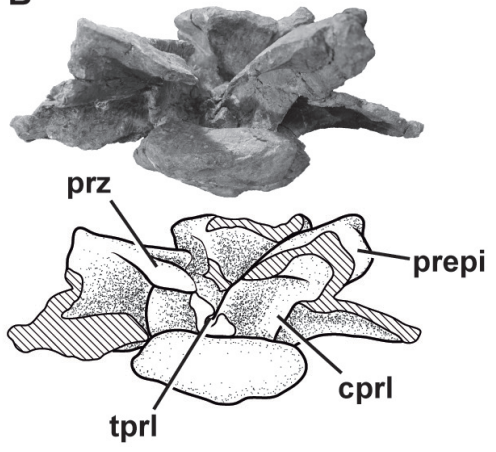

C

poz
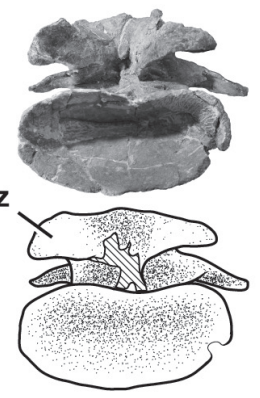

D
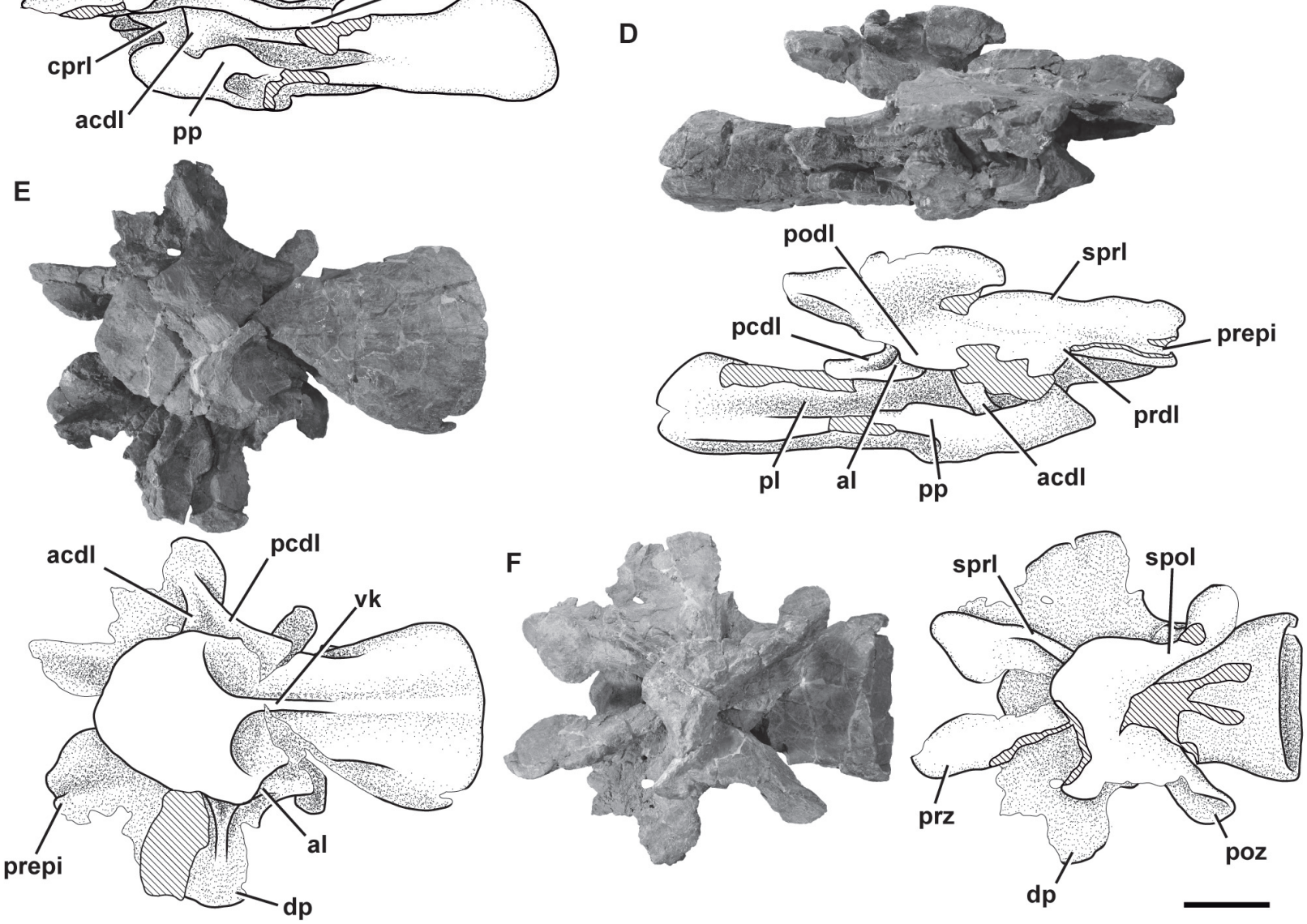

FIGURE 11. Middle-posterior cervical vertebra of Bagualia alba (MPEF-PV3408) in left lateral (A), anterior (B), posterior (C), left lateral (D), ventral (E), and dorsal (F) views. Abbreviations: acdl, anterior centrodiapophyseal lamina; al, accessory lamina; cprl, centroprezygapophyseal lamina; dp, diapophysis; pcdl, posterior centrodiapophyseal lamina; pl, pleurocoel; podl, postzygodiapophyseal lamina; poz, postzygapophysis; pp, parapophysis; prdl, prezygodiapophyseal lamina; spol, spinopostzygapophyseal lamina; sprl, spinoprezygapophyseal lamina; tprl, intraprezygapophyseal lamina; vk, ventral keel. Hatched pattern represents broken surfaces and grey form represents sediment. Scale bar equals $10 \mathrm{~cm}$.

ses are low and are restricted to the anteroventral corner of the centrum. They are ovoid in shape and directed ventrolaterally. Large, undivided pleurocoels occupy most of the lateral surface of the centrum (Figure 11A, D). As in the rest of the cervical vertebrae, the pleurocoels are defined by their anterior, dorsal, and ventral margins, being well excavated anteriorly and becoming shallow posteriorly. The transverse process is anteroposteriorly long and low. Both transverse processes are nearly complete, lacking only their posterior margins. These processes are flat, lateromedially longer than dorsoventrally high (due to the dorsoventral compression), and oriented laterally and slightly 
ventrally. The transverse process is supported ventrally by a short, thick ACDL and a long PCDL. The $A C D L$ is ventrally positioned in the transverse process and dorsally reaches the PCDL at the distal tip of the diapophysis. The PCDL is as broad as the $A C D L$, and extends almost horizontally from the ventral margin of the transverse process to the posterodorsal margin of the centrum without reaching the posterior articular surface. The ACDL, PCDL, and the dorsal border of the pleurocoel form the anterodorsal, posterodorsal, and ventral edges of the CDF, respectively. The CDF is triangular and dorsoventrally crushed. On the right side, there is an accessory lamina below the transverse process just anterior to the PCDL (Figure 11D). This sheet could be homologous with the accessory lamina seen in the middle cervical vertebrae (MPEF-PV $3301 / 16$, MPEF-PV 11040/2), but it is oriented posteroventrally. Also, this lamina is only seen on one side, so it is considered as a particular variation in the lamination of this vertebra.

A marked PRDL runs from the ventrolateral surface of the prezygapophysis to the diapophysis, projecting horizontally. In lateral view, the prezygapophyseal processes are horizontal and extend anteriorly, surpassing the anterior articular surface of the centrum. The prezygapophyses are flat and oval in shape, with the major margin oriented dorsomedially (Figure 11F). On the ventrolateral surface of the prezygapophysis there is a marked preepipophysis, as in the anterior and mid-cervical vertebrae. Both prezygapophyses are linked by very thin paired TPRLs that run ventrally (Figure 11B). No sTPRL is visible due to damage. The neural canal is crushed and broken anteriorly, and posteriorly is compressed. It is small and ovoid in shape. A stout CPRL runs vertically from the ventral base of the prezygapophyseal process, ventral to the laterodorsal corner of the centrum. The CPRL defines, with the ACDL and the PRDL, a deep and triangular PRCDF. This fossa has the shape of an inverted triangle with its base oriented dorsally. Anteriorly, the TPRL and the CPRL define a long, dorsoventrally oriented CPRF that extends along the entire ventral surface of the prezygapophyseal process. The postzygapophyses are oriented laterally without reaching the level of the posterior articular surface of the centrum, unlike the more anterior cervical vertebrae (Figure 11C). Despite being very deformed, the postzygapophyses of this element are dorsally inclined and they do not reach the posterior articular surface of the centrum, as occurs in most sauropods (e.g., Patagosaurus, Mamenchisaurus; PVL 4170; Ouyang and Ye, 2002, figure 13). No marked CPOLs are recognized, as in the more anterior cervical vertebrae. The complete left PODL extends from the posterior margin of the diapophyses to the ventrolateral surface of the postzygapophysis. This lamina is oriented posterodorsally, defining the dorsal edge of a POCDF that is dorsoventrally crushed.

The neural spine is dorsoventrally crushed and lacks the left anterolateral portion of its distal end. The neural spine maintains the trend of shortening throughout the cervical series, and is lateromedially wider than anteroposteriorly long (Figure $11 \mathrm{~F}$ ). The prezygapophyses are linked with the neural spine by long SPRLs. These incomplete laminae are oriented dorsomedially from the dorsomedial edge of the prezygapophyses to the distal tip of the neural spine where they widen laterally. Both SPRLs bound the SPRF, in which no signs of a PRSL are observed. The slightly anterodorsally directed SPOLs are as long as the SPRLs and extend from the postzygapophyses up to the dorsal end of the neural spine. They are parallel to each other and form the lateral walls of the SPOF, which extends across nearly the whole posterior surface of the neural spine. As with the PRSL, the POSL is absent. The undamaged posterior surface of the neural spine appears to be rough in texture.

MPEF-PV 3327. This element is poorly preserved and very deformed. In anterior view the vertebra is inclined towards the left at about 45 degrees. The vertebra preserves a portion of its centrum and the right transverse process. In the ventral surface of the centrum there is a keel restricted to the anterior zone (where it is more marked) that vanishes posteriorly, occupying two thirds of the length of the centrum. On both sides of the anterior part of the centrum, there are two deep concavities on each side of the keel. The pleurocoels are deep without division. The septum that separates the pleurocoels is rough, and does not communicate with internal chambers. The transverse process is directed downwards, perhaps by the general deformation of the vertebra. The neural canal is only visible in posterior view, as it is deformed.

MPEF-PV 3349. This vertebra is incomplete (Figure 12). The centrum is opisthocoelous with its posterior articular surface strongly concave. Although deformed, the centrum is notably shorter than previous vertebrae (El: 2; see Table 3 in Appendix 1), in accordance with a posterior position for this element. On the ventral surface of the centrum, there is a keel extending for nearly the first half of the total length then fading posteriorly (Figure 12E). Only the bases of the parapophyses 


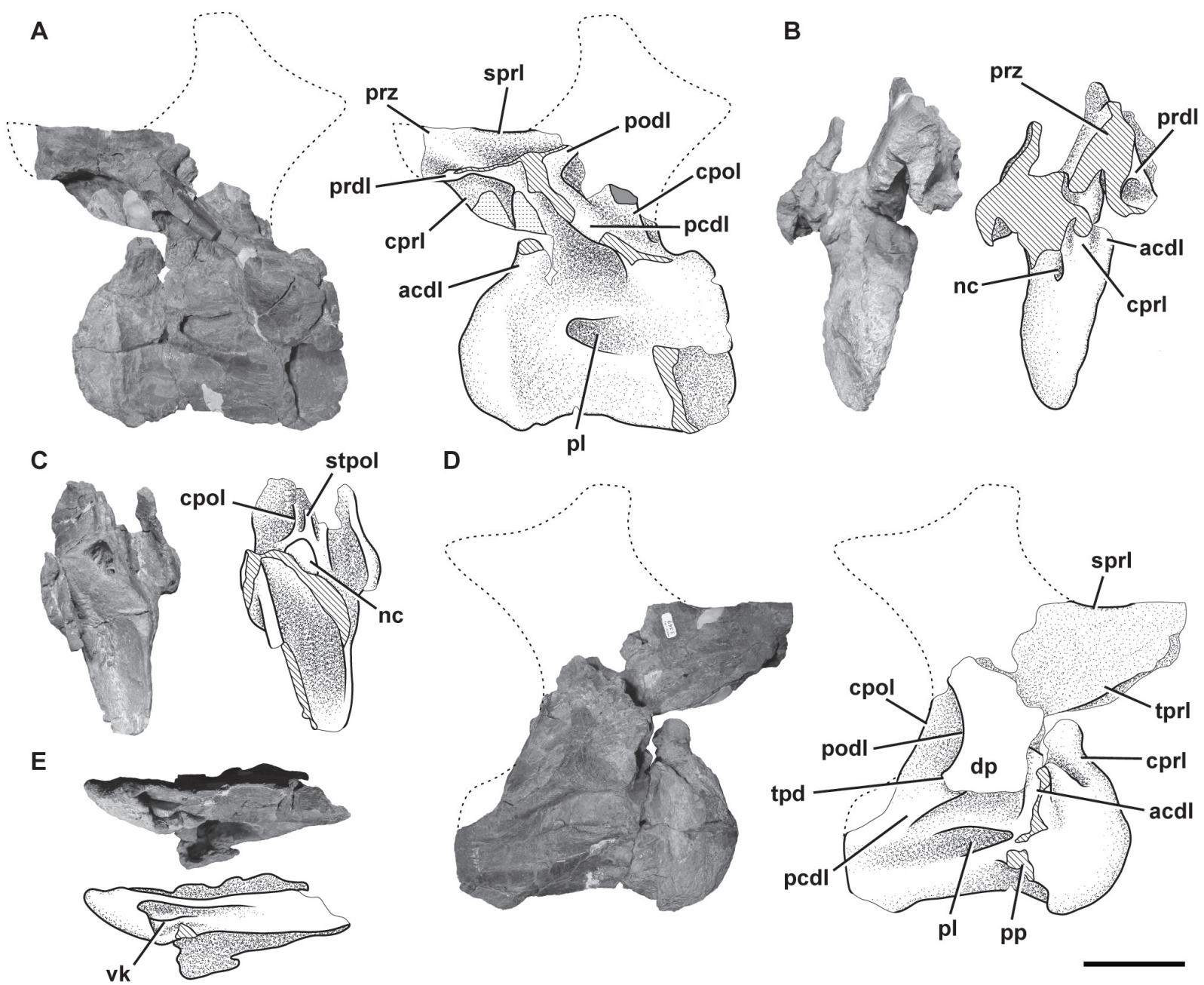

FIGURE 12. Posterior cervical vertebra of Bagualia alba (MPEF-PV 3349) in left lateral (A), anterior (B), posterior (C), right lateral (D), and ventral (E) views. Abbreviations: acdl, anterior centrodiapophyseal lamina; cpol, centropostzygapophyseal lamina; cprl, centroprezygapophyseal lamina; dp, diapophysis; nc, neural canal; pcdl, posterior centrodiapophyseal lamina; pl, pleurocoel; podl, postzygodiapophyseal lamina; pp, parapophysis; prdl, prezygodiapophyseal lamina; prz, prezygapophysis; sprl, spinoprezygapophyseal lamina; stpol, simple intrapostzygapophyseal lamina; tpd, triangular process of the diapophysis; tprl, intraprezygapophyseal lamina; vk, ventral keel. Dashed line for the reconstructed parts. Hatched pattern represents broken surfaces and grey form represents sediment. Scale bar equals $10 \mathrm{~cm}$.

are preserved. They are rounded and restricted to the anteroventral corner of the centrum (Figure 12D). The lateral surfaces present distinct pleurocoels that are similar in shape to those seen in more anterior cervical vertebrae, with the anterior, dorsal, and ventral edges well-marked and the posterior one absent (Figure 12A, D). In posterior view, the neural canal is triangular in shape, and in anterior view is slit-shaped due to the lateromedial compression of this element (Figure 12C). The right transverse process is nearly complete. The transverse processes are positioned higher on the vertebra than in more anterior cervicals. They are ventrally supported by a broad, vertically oriented ACDL and a posteroventrally directed PCDL (at an angle of 45 degrees). The ACDL extends downwards from the anterior margin of the diapophysis to the anterodorsal corner of the centrum. The PCDL runs from the posterior edge of the diapophysis and reaches the posterior articular surface of the centrum. The posteriorly oriented triangular process, also present in middle cervical vertebrae, is incompletely preserved but well developed. Both ACDL and PCDL define a triangular and deep CDF. 
Only the left prezygapophyseal process is preserved. It is nearly horizontal, medially flat, and bears a small portion of the prezygapophysis. The PRDL runs from the lateral surface of the prezygapophyseal process to the anterodorsal margin of the transverse process, with a similar angle as the PCDL. On the lateral surface of the distal end of the prezygapophyseal process, there is a small, deep fossa on the PRDL. The incomplete left CPRL is broad and runs ventromedially from the ventral surface of the prezygapophysis to the anterodorsal surface of the centrum, where it reaches the ACDL (Figure 12B). Although both postzygapophyses are absent, it is possible to recognize some postzygapophyseal laminae. Only the bases of the PODLs are preserved. The left anterior portion of the PODL is thin. The CPOL, which was not developed in the anterior and mid-cervical vertebrae, can be recognized in this posterior cervical. The CPOLs run dorsally from each side of the roof of the neural canal. Right above the roof of the neural canal there is a thin, vertical STPOL (Figure 12C). This lamina and the CPOLs define small, paired, vertical, elliptic CPOF above the neural canal. Between the PODL, PCDL, and CPOL there is a deep POCDF on the left lateral side.

The proximal portion of the left SPRL extends horizontally backwards and defines, with the PRDL and PODL, a deep SDF above the transverse process. The SPDL is absent in this vertebra.

MPEF-PV 3348. This vertebra is very deformed and fragmented. In anterior view, the left parapophysis is anteroventrally oriented, and the posterior portion of the vertebra is curved towards the right. This deformation was caused by compressive force on the right anterolateral side of this element. The centrum is complete and has a low El (0.9; see Table 3 in Appendix 1). Both sides of the centrum have well-defined pleurocoels that occupy almost their entire length and do not connect with internal chambers or other pneumatic cavities. The septum separating the pleurocoels is smooth in texture, differing from those of the holotype specimen where this surface was rough. On the anterior part of the pleurocoel, there is a marked circular depression. The parapophyses are well developed, and the right has the proximal portion of its cervical rib fused with it, indicating a certain degree of maturity in this specimen. As in MPEF-PV 11040/2, the dorsal surface of the parapophysis is excavated and separated from the pleurocoel by a slight elevation of the bone. The proximal portion of the rib has the capitular and tubercular processes form a high angle (almost 180 degrees), indicating the posterior position of this vertebra in the neck, where the diapophysis becomes higher in the neural arch. The neural canal is triangular anteriorly and posteriorly, which is a product of deformation. The diapophyses are well developed, with their long, welldefined ACDL reaching the anterior articular face of the centrum, while the PCDL connects the diapophysis to the posterior articular surface. The right transverse process has the triangular process described for more anterior cervical vertebrae. This process is better developed in this specimen than in any of the more anterior cervical vertebrae described. Below the transverse process there is a deep CDF. The PRDL is well developed, and defines a deep PRCDF in conjunction with the $A C D L$ and the CPRL. Underneath the right PODL, there is a deep POCDF. Both prezygapophyses are well preserved, although very deformed. The CPRLs are well developed, and are long and form the walls of the neural canal. The TPRL is wider than in the anterior vertebrae, contacts the roof of the neural canal, and forms a deep CPRF with the CPRL. The postzygapophyses are highly developed. Their articular surfaces are flat and sheetlike. On the dorsal surface of the right postzygapophyseal process, there is a well-developed epipophysis as an extension of the SPOL, which reaches the posterior margin of the postzygapophysis without surpassing it.

Observing this, we can trace the development of the epipophysis along the cervical series. As described, the axis of Bagualia has epipophyses that occupy the entire dorsal surface of the postzygapophysis and extend beyond the posterior margin of it as a prong (sensu D'Emic, 2012). This epipophysis is separated from the SPOL, which is medial to the postzygapophysis. In the anterior and middle cervicals, the epipophysis continues to be developed on the dorsal surface of the postzygapophysis, but there is a slight union between it and the SPOL, which now takes a more lateral position. Towards the posterior cervicals, the SPOL becomes more lateral and extends over the dorsal surface of the postzygapophyses, forming the epipophysis. In these elements, the ridge on the dorsal surface of the postzygapophysis observed in the anterior vertebrae does not exist, because now the epipophysis is formed by the SPOL on the dorsal surface of the postzygapophysis. In the most posterior cervicals, the epipophysis forms a laminar posterior extension of the SPOL just above the postzygapophysis without contacting it, as observed in other sauropods (e.g., Shunosaurus, 


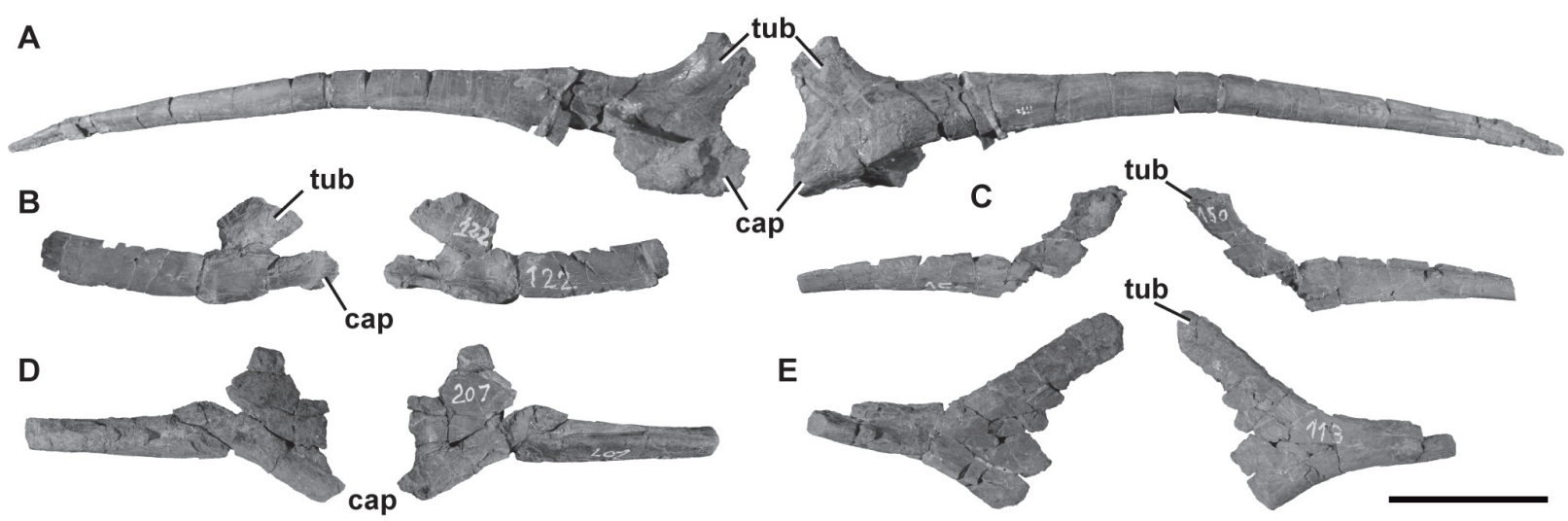

FIGURE 13. Cervical ribs of Bagualia alba. A, MPEF-PV 11058 in medial and lateral views; B, MPEF-PV 11054 in lateral and medial views; C, MPEF-PV 11057 in medial and lateral views; D, MPEF-PV 11056 in lateral and medial views; E, MPEF-PV 11055 in medial and lateral views. Abbreviations: cap, capitulum; tub, tuberculum. Scale bar equals 10 $\mathrm{cm}$.

Patagosaurus, Spinophorosaurus, Jobaria; ZDM T5401; PVL 4170; GCP-CV 4229, NMB 1699 R; MNN TIG 3-5). This would indicate that the epipophysis shows greater development on the dorsal surface of the postzygapophysis in the anterior elements of the neck, whereas posteriorly in the series it develops like a prong from the margin of the postzygapophysis and migrates dorsally to be a posterior extension of SPOL in the last cervicals.

The neural spine is lateromedially wider (by about six times) than anteroposteriorly long. Although this relation may be exaggerated by deformation in this vertebra, it is clear that the neural spines of the posterior-middle and posterior cervical vertebrae are wider than long. This feature and the elongation index of the centrum indicate that this vertebra could belong to a posteriormost position in the cervical series. The right SPOL is complete and exposed in both lateral views due to poor preservation of the left one. This lamina is broad and runs from the dorsal margin of the postzygapophysis to the distal posterior edge of the neural spine.

\section{Cervical Ribs}

Several cervical ribs were recovered from the Cañadón Bagual locality, which were found isolated and without clear association to vertebral elements. Because most of the cervical ribs are represented by fragments of different sizes, this description is based mainly on the most complete elements (Figure 13). Taking into account that the angle between capitulum and tuberculum (and their lengths) varies along the neck (following the separation between parapophyses and diapophyses; Carballido and Sander, 2014) some elements can be identified as anterior cervical ribs (MPEFPV 11058, MPEF-PV 11054) and others as posterior cervical ribs (MPEF-PV 11055, MPEF-PV 11056, MPEF-PV 11057). The ribs are tetraradiate proximally, with a well-developed tuberculum, capitulum, and anterior process, and a long, slender shaft directed posteriorly as in most sauropods (Upchurch et al., 2004). The most complete cervical rib is MPEF-PV 11058, which is longer than any preserved cervical centrum (Figure 13A). Therefore, it seems that the cervical ribs of Bagualia were around twice the length of the centrum, as in most sauropods except diplodocoids (Wilson and Sereno, 1998; Upchurch et al., 2004). MPEF-PV 11058 has its proximal portion well preserved. In this rib, the anterior process is incomplete, having a triangular shape in cross section with the major axis oriented dorsoventrally. The tuberculum is vertically oriented, blade-shaped (mediolaterally flattened), and lacking its articular surface with the diapophysis. The capitulum is more robust than the tuberculum but has the same length. This process is oriented anteriorly, slightly surpassing the anterior margin of the tuberculum. In cross section, the capitulum has a semicircular shape, being flat ventrally and convex dorsally. The articular surface with the parapophysis is circular and slightly concave. The angle between capitular and tubercular processes is about 60 degrees, similar to that of the anterior cervical ribs of other sauropods (e.g., Spinophorosaurus, Camarasaurus; GCP-CV 4229; Mclntosh et al., 1996). In most posterior cervical ribs this angle increases to be about 90 degrees (Figure 13B-E). At the base of the tuberculum, there is an elongate concavity subdivided by a short, posteroventrally oriented lamina. This con- 
cavity and its division are also present in MPEF-PV 11054 (Figure 13B), and it could be an incipient, albeit not truly, pneumatic structure. The shaft is around $37 \mathrm{~cm}$ long. The ventral surface of the shaft is proximally flat, becoming concave as a sulcus that fades near the half of the full anteroposterior length of the rib. Distally, the shaft becomes rounded in cross section.

\section{Dorsal Vertebrae}

Nine dorsal vertebrae referable to Bagualia alba are present, described as eight different specimens, seven represented by isolated elements (MPEF-PV 3403; MPEF-PV 3405; MPEF-PV 3343; MPEF-PV 11000; MPEF-PV 11012; MPEF-PV 11023; MPEF-PV 11027), and one (MPEF-PV 11011) being part of a single specimen represented by the last two dorsal vertebrae, an incomplete sacrum with a pubis, and the first 10 caudal vertebrae. Preserved dorsal elements represent the anterior, middle, and posterior sections of the dorsal series. As in the cervical vertebrae, the description is following an anterior-posterior anatomical direction. The preserved dorsal vertebrae have greater development of their zygapophyses and apophyses than cervical vertebrae, as well as of the bony laminae that unite them (see Table 2 in Appendix 1). The parapophyses ascend in this axial region from the dorsoventral mid-length of the centrum in the anteriormost dorsal vertebrae (Carballido and Sander, 2014), to being completely located in the neural arch from the third dorsal onward. This change in the position of the apophyses is found in all sauropods and is especially important for recognizing the position of dorsal vertebrae (Upchurch et al., 2004). The neural spines are higher than in the cervical vertebrae, so that the SPRLs and SPOLs have a greater angle.

MPEF-PV 11023. Due to the low position of the parapophyses and the lack of the hyposphenehypantrum system (Carballido and Sander, 2014), this vertebra is interpreted as the first or second dorsal (Figure 14). The centrum is strongly opisthocoelous, lateromedially compressed due to deformation, and, as in most sauropods, lacks the ventral keel (e.g., Spinophorosaurus; GCP-CV4229). The ventral keel is present in some eusauropods (e.g., Patagosaurus; Holwerda et al., 2021) and derived taxa (e.g., Diplodocus, Haplocanthosaurus; Upchurch et al., 2004). The El is around 1.9 (see Table 4 in Appendix 1). The shape of the pleurocoels resembles those present in cervical vertebrae (see above). The parapophyses are located in the centrum, at the anterodorsal corner of the pleurocoels (Figure 14A, E). They are circular and concave to receive the capitulum of the dorsal rib. As the parapophyses are located in the centrum, parapophyseal laminae (ACPL, PCPL, PRPL, and PPDL) are not present. In anterior view, the prezygapophyses are inclined ventromedially at an angle of 45 degrees. The articular surface is flat and elliptical in shape, with the major axis oriented slightly posteromedially with respect to the sagittal plane. The CPRL is a long, robust lamina running from the anteroventral tip of the prezygapophyses to the anterodorsal surface of the centrum. The ventral end of the CPRL is connected to the anterior end of the ACDL. As in the posterior cervical vertebrae, there is a STPRL that connects both TPRLs with the roof of the neural canal, although it is longer than that of the cervical vertebrae due to the increased dorsoventral height of the neural arch (Figure 14B). The presence of a TPRL is common in the anteriormost dorsal vertebrae of sauropods, which lack the hypantrum (Wilson, 1999; Carballido and Sander, 2014). Between the CPRL and the TPRL + sTPRL there is a deep CPRF. A broad PRDL runs from the lateral surface of the prezygapophysis to the diapophysis. In both prezygapophyseal processes there is a ridge extending anteriorly from the PRDL, which could be observed in at least the first two dorsal vertebrae of other eusauropods (e.g., Shunosaurus, Patagosaurus, Mierasaurus, Tendaguria; IVPP V 9065; MACN-CH 936; Royo Torres et al., 2017: figure AB; Mannion et al., 2019a, figure 19) and the titanosauriform Euhelopus (PMU 233). This anterior extension of the PRDL appears to be homologous with the pre-epipophysis present in cervical vertebrae, or a relic of it within the anteriormost dorsal vertebrae. This feature is not present in the rest of the preserved dorsal vertebrae, which correspond to the middle and posterior region of the axial series, so can be considered as a structure that generally appears in the middle cervical vertebrae, reaches its maximum development towards the posterior cervicals, and persists in the first dorsal vertebra.

The postzygapophyses are elliptical in shape and oriented ventromedially. Both TPOLs are longer than TPRLs (Figure 14D). Due to poor preservation, the presence of a sTPOL is unknown. The CPOL is only preserved on the left side as a short lamina lateral to the TPOL, as in Patagosaurus (MACN-CH 936). The PODL is as long as the PRDL and runs parallel to the SPRL from the anterior margin of the postzygapophysis to the dorsal surface of the diapophysis. The PODL, PCDL, and 

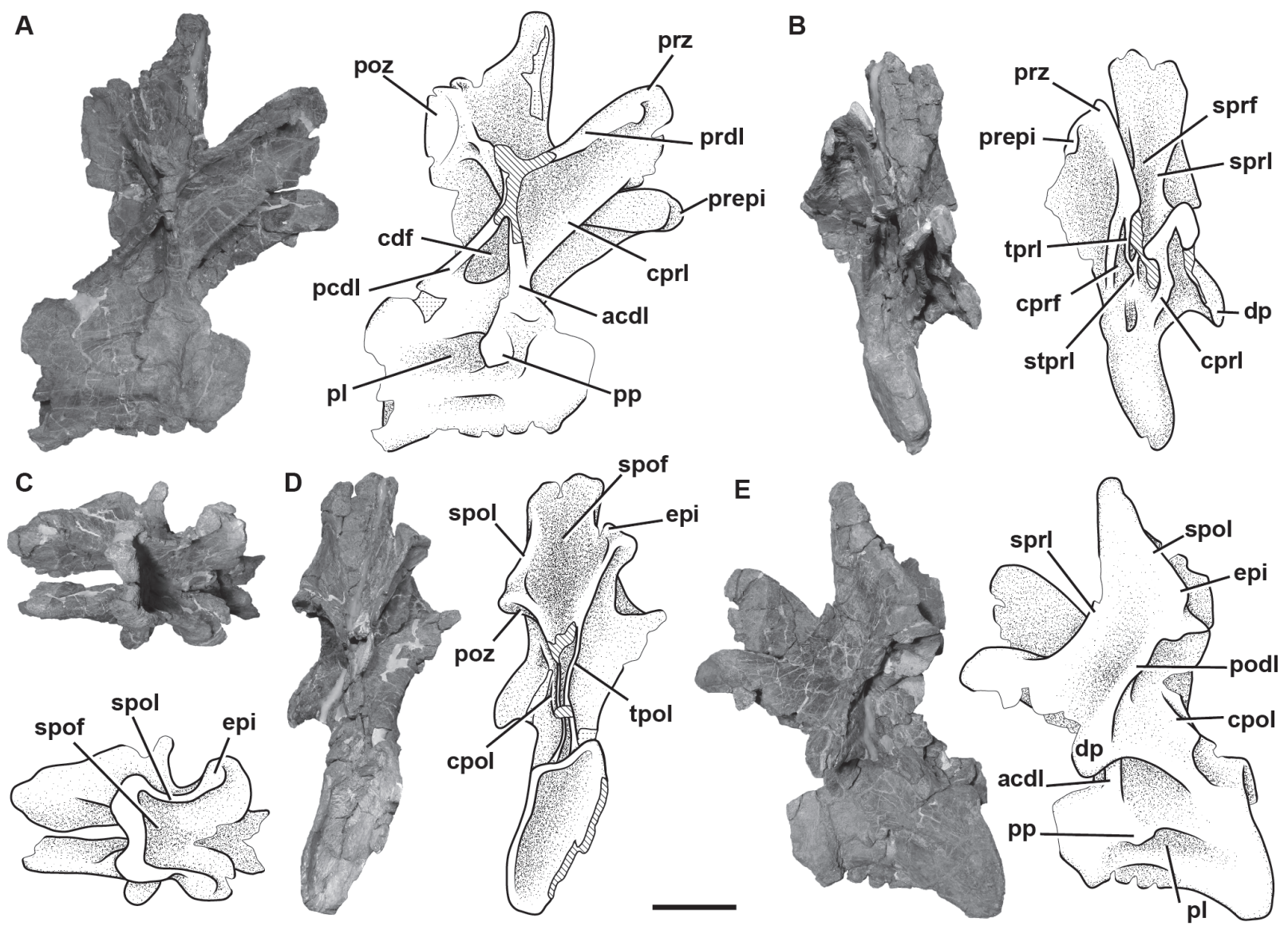

FIGURE 14. Anterior dorsal vertebra of Bagualia alba (MPEF-PV 11023) in right lateral (A), anterior (B), dorsal (C), posterior (D), left lateral (E) views. Abbreviations: acdl, anterior centrodiapophyseal lamina; cdf, centrodiapophyseal fossa; cpol, centropostzygapophyseal lamina; cprf, centroprezygapophyseal fossa; cprl, centroprezygapophyseal lamina; dp, diapophysis; epi, epipophysis; pcdl, posterior centrodiapophyseal lamina; pl, pleurocoel; podl, postzygodiapophyseal lamina; poz, postzygapophysis; pp, parapophysis; prdl, prezygodiapophyseal lamina; prepi, pre-epipophysis; prz, prezygapophysis; spof, spinopostzygapophyseal fossa; spol, spinopostzygapophyseal lamina; sprf, spinoprezygapophyseal fossa, sprl, spinoprezygapophyseal lamina; stprl, single intraprezygapophyseal lamina; tpol, intrapostzygapophyseal lamina; tprl, intraprezygapophyseal lamina. Hatched pattern represents broken surfaces. Scale bar equals $10 \mathrm{~cm}$.

TPOL define a deep POCDF as in other sauropods (e.g., Spinophorosaurus, Patagosaurus). On the anterodorsal corner of this fossa there are at least two shallow concavities separated by a thin lamina, which is also present in Moabosaurus (Britt et al., 2017, figure 19G). As in posterior cervical vertebrae, an epipophysis is present, although in this vertebra this is an extension of the SPOL above the postzygapophysis without exceeding its posterior margin (Figure 14D, E). Furthermore, this lamina curves laterally above the postzygapophysis. The presence of epipophyses in anteriormost dorsal vertebrae is rare, being present in the first dorsal vertebrae of a few taxa (e.g., Yunnanosaurus, Moabosaurus, NHMUK 1871, Euhelopus; Lu et al., 2007; Britt et al., 2017, figure 19; Mannion, 2019;
Wilson and Upchurch, 2009) but absent in most sauropods, such as Patagosaurus (PVL 4170; $\mathrm{MACN}-\mathrm{CH}$ 936). The transverse process is almost complete on the left side, being large and slightly oriented anteroventrally. The dorsal surface of the transverse process is flat and smooth. This process lacks any fossae on its anteroventral surface, which are seen in Moabosaurus (Britt et al., 2017). The diapophysis is rough and rounded. The ACDL is nearly vertical and runs from the ventral surface of the transverse process to the anterodorsal surface of the centrum, where this is fused with the CPRL. Between the CPRL, ACDL, and PRDL, there is a deep PRCDF. The PCDL runs from the posterior margin of the transverse process posteroventrally to the posterodorsal surface of the cen- 
trum close the articular surface. Both ACDL and PCDL enclose a deep triangular CDF, which is separated from its opposing fossa by a thin septum of bone, as in Patagosaurus (Holwerda et al., 2021). Like Spinophorosaurus (CP-CV-4229) and Patagosaurus (MACN-CH 936), between the transverse process and the neural spine there is a rounded, undivided, very deep SDF. A divided SDF is present in some sauropods (e.g., Mamenchisaurus, Camarasaurus, Brachiosaurus; Wilson et al., 2011).

The neural spine is vertical, and around 2.6 times wider lateromedially than anteroposteriorly long (Figure 14C). Its anteroposterior length is 13\% of the full anteroposterior length of the centrum. Both anterior and posterior surfaces are rough, and although no clear POSL and PRSL are present, the rough surface is in the place of these single laminae. The SPOL extends to the distal posterior tip of the spine and encloses a tall, deep SPOF. The SPRL extends at least for half of the dorsoventral length of the spine. The lateral margins of the neural spine are expanded slightly close to the tip, giving it a convex shape in anterior or posterior views. This morphology clearly differs from that of other taxa, which have an aliform process (see below).

MPEF-PV 11012. Isolated dorsal centrum dorsoventrally compressed so that both articular surfaces are exaggeratedly concave. This centrum preserves a small portion of the base of the neural arch. The centrum does not have a ventral keel. The lateral fossa is elliptical in shape, with its greater axis oriented anteroposteriorly and, in contrast to the anterior dorsal vertebra, only the ventral margin is distinct. This fossa is located a little closer to the anterior articular surface than the posterior one and restricted to the centrum.

MPEF-PV 3343. Isolated dorsal centrum with a small portion of the neural arch. This vertebra is dorsoventrally crushed so that the posterior articular surface and lateral fossae are exaggeratedly deepened due to taphonomic deformation. As in the other dorsal vertebrae, there is no ventral keel. The lateral fossae are elliptical, with their major axis oriented anteroposteriorly.

MPEF-PV 3405. Centrum with a small portion of the neural arch crushed lateromedially. Its centrum is amphicoelous and lacks a ventral keel. The left lateral fossa is ovoid in shape and is placed near the anterodorsal corner of the centrum.

MPEF-PV 3403. Centrum dorsoventrally compressed. This centrum has a posterior articular sur- face deeper than the anterior one and does not have a ventral keel.

MPEF-PV 11027. This is the fragmentary, isolated neural arch of a mid-posterior dorsal vertebra (Figure 15). This vertebra was recovered in association with a posterior caudal vertebra that bears the same collection number, although cannot be confidentially assigned to the same specimen. The dorsal element is solely represented by the left ventral section of the neural arch. The hyposphene-hypantrum, if it was present, is not preserved. The parapophysis has a semicircular articular surface and was separated from the dorsal margin of the centrum. The PCPL curves and becomes thin posteroventrally, whereas the ACPL is not recognizable (Figure 15B, D). As the parapophysisis located in the neural arch, the lamina that runs anteroventrally from the diapophysis is the PPDL, which connects the diapophysis and the parapophysis at an angle of some 12 degrees. The right prezygapophysis has a flat, oval-shaped surface, with its lateral margin anteroventrally inclined (Figure 15C). In anterior view, the prezygapophyses are inclined ventromedially, forming an angle of approximately 45 degrees. The short PRPL is thick and projects posteroventrally from the base of the prezygapophysis to the anterodorsal margin of the parapophysis (Figure 15B).

As in MPEF-PV 11023, there are no fossae on the anteroventral surface of the transverse process. The diapophysis is 3.4 times anteroposteriorly longer than dorsoventrally high (Figure 15A, C). In both its anterior and posterior edges, the diapophysis expands dorsoventrally, giving its dorsal edge a concave shape. The PRCDF is oval, long, and oriented anteroposteriorly below the PRDL. Close to its mid-length, the PRDL is divided into two laminae, a very thin dorsal lamina and a thicker ventral one that connects the prezygapophysis with the anteromedial margin and the ventral surface of the transverse process, respectively (Figure 15D). The PCDL arise under the transverse process as a short single lamina. The PPDL anteriorly and PCDL posteriorly enclose a triangular CDF (Figure 15D). The SPDL, widespread in many other sauropods (e.g., Tazoudasaurus, Omeisaurus, Mierasaurus, Moabosaurus; Allain and Aquesbi, 2008; He et al., 1988, figure 28; Royo Torres et al., 2017; Britt et al., 2017), is absent in dorsal vertebrae of Bagualia (Figure 15C). The eusauropod Spinophorosaurus appears to have a lateral spinal lamina posterior to the SPRL that could be a reduced SPDL, which has been observed by Tschopp et al. (2015) in the specimen NMB-1699-R. This lamina 

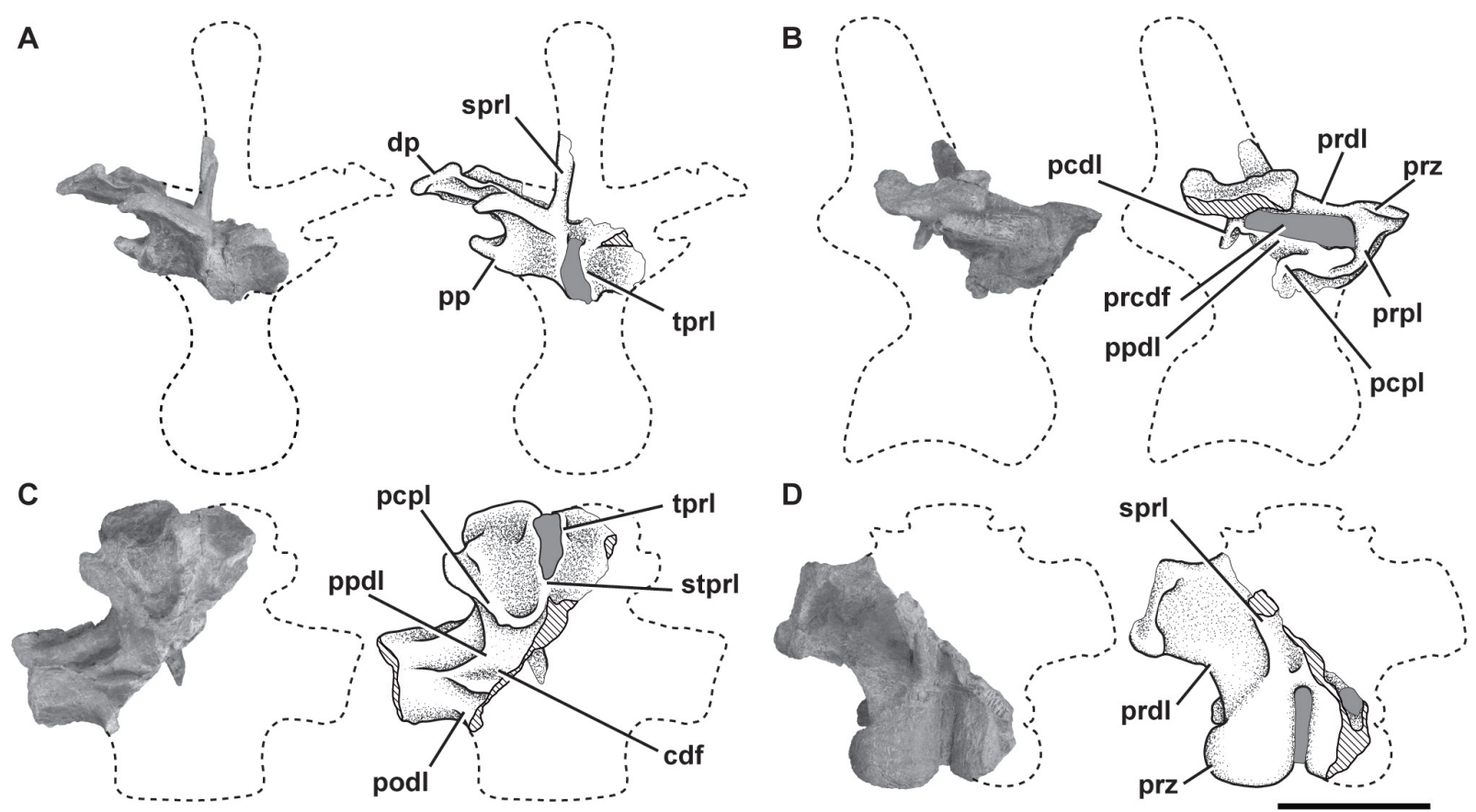

FIGURE 15. Mid-posterior dorsal vertebra of Bagualia alba (MPEF-PV 11027) in anterior (A), right lateral (B), ventral (C), and dorsal (D) views. Abbreviations: cdf, centrodiapophyseal fossa; dp, diapophysis; pcdl, posterior centrodiapophyseal lamina; pcpl, posterior centroparapophyseal lamina; podl, postzygodiapophyseal lamina; pp, parapophysis; ppdl, paradiapophyseal lamina; prcdf, prezygapophyseal centrodiapophyseal fossa; prdl, prezygodiapophyseal lamina; prpl, prezygoparapophyseal lamina; prz, prezygapophysis; sprl, spinoprezygapophyseal lamina; stprl, single intraprezygapophyseal lamina; tprl, intraprezygapophyseal lamina. Dashed line for the reconstructed parts. Hatched pattern represents broken surfaces. Scale bar equals $10 \mathrm{~cm}$.

also appears to be present in the cervical vertebrae in the Spinophorosaurus specimen GCP-CV 4229, where it is marked on the lateral surface of the neural spine, and distally forms a protuberance (GCPCV 4229/HB 4-7).

MPEF-PV 11011. Two mid-posterior dorsal neural arches (probably 5 and 6 ) were found closely associated with a partial sacrum, pubis, and the first 10 caudal vertebrae, so are considered part of the same specimen. Both neural arches lack the distal portion of their neural spines (Figure 16). Whereas the anterior neural arch lacks the entire anterior portion from the level of the diapophyses, the posterior one is almost complete. Due to the articulation between both arches, the hyposphene cannot be visualized. The parapophyses, preserved in the posterior neural arch, are elliptical in shape, with the major axis oriented dorsoventrally (Figure 16A). The short, vertically oriented ACPL is visible on the right lateral side, running downward from the ventral edge of the parapophysis. The PCPL is developed on the left lateral side and is posteroventrally oriented at an angle of 45 degrees, fading posteriorly without reaching the PCDL or the centrum (Figure 16D). A short, thick PRPL runs verti- cally from the lateral edge of the prezygapophysis to the parapophysis. The PRDL, only preserved in the posterior vertebra (Figure 16A, D), connects the prezygapophysis with the diapophysis. This lamina, in conjunction with the PRPL and the PPDL, defines a deep PRCDF which is oriented posterodorsally. The prezygapophyses (solely preserved in the more posterior neural arch of the two preserved) are medially obscured by the postzygapophyses of the anterior vertebra, as they are in articulation, although they seem to be dorsoventrally long and oriented dorsomedially. Although the hypantrum is obscured by the hyposphene of the anterior vertebra, its presence results in the absence of the TPRL, a common change in all sauropods (Wilson, 1999). The postzygapophyses are hoof-shaped and have flat surfaces. A weakly developed hyposphene is present. The hyposphene is slightly triangular in shape, with its major axis oriented vertically and with an incipient ventral expansion (Figure 16B). Its posterior surface is concave and smooth, and below it runs two thin mCPOLs, which bound the neural canal. The presence of both a hyposphene-hypantrum and the $\mathrm{mCPOLs}$ is common in posterior dorsal vertebrae 


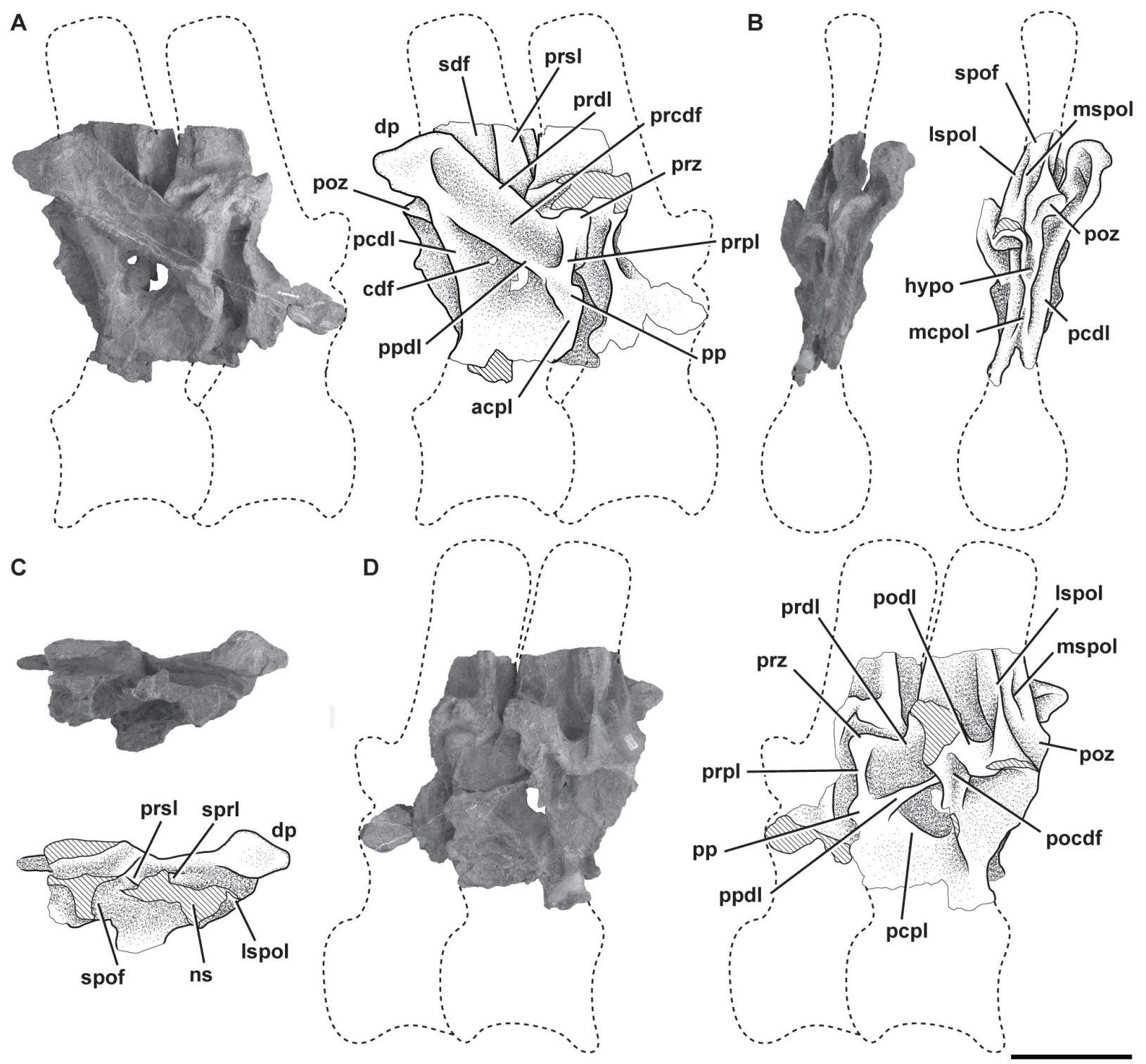

FIGURE 16. Middle-posterior dorsal neural arches of Bagualia alba (MPEF-PV 11011) in right lateral (A), posterior (B), dorsal (C), and left lateral (D) views. Abbreviations: acpl, anterior centroparapophyseal lamina; cdf, centrodiapophyseal fossa; dp, diapophysis; hypo, hyposphene; Ispol, lateral spinopostzygapophyseal lamina; mcpol, medial centropostzygapophyseal lamina; mspol, medial spinopostzygapophyseal lamina; ns, neural spine; pcdl, posterior centrodiapophyseal lamina; pcpl, posterior centroparapophyseal lamina; pocdf, postzygapophyseal centrodiapophyseal fossa; podl, postzygodiapophyseal lamina; poz, postzygapophysis; pp, parapophysis; ppdl, paradiapophyseal lamina; prcdf, prezygapophyseal centrodiapophyseal fossa; prdl, prezygodiapophyseal lamina; prpl, prezygoparapophyseal lamina; prsl, prespinal lamina; prz, prezygapophysis; sdf, spinodiapophyseal fossa; spof, spinopostzygapophyseal fossa; spol, spinopostzygapophyseal lamina; sprl, spinoprezygapophyseal lamina. Dashed line for the reconstructed parts. Hatched pattern represents broken surfaces. Scale bar equals $10 \mathrm{~cm}$.

of other sauropods (Carballido and Sander, 2014). The transverse process is large and oriented posterodorsally. The dorsal orientation is exaggerated due to lateromedial compression of the bones. The diapophysis is rhomboid in shape, with the largest axis oriented anteroposteriorly. The PPDL is a thin, long lamina that arises below the transverse pro- cess from its union with the PCDL. This lamina replaces the ACDL of the cervical and most anterior dorsal vertebrae in most sauropods (e.g., Camarasaurus, Amargasaurus; Osborn and Mook, 1921; Salgado and Bonaparte, 1991, MACN-N 15). The PCDL runs almost vertically from the diapophysis to the centrum. The PCDL, PPDL, and the 
PCPL define deep CDFs on each side of the arch (Figure 16A, D). Left and right CDFs are separated by a thin septum, which is broken due to its fragility. A short, thick PODL develops from the diapophysis and contacts the postzygapophysis anteroventrally. On the left side of the posterior vertebra, there is a short lamina that contacts the PODL with the PCDL. This accessory lamina divides the POCDF in two small fossae (Figure 16D). This subdivision of the POCDF by laminae is also present in other sauropods (e.g., Moabosaurus, Camarasaurus, Rapetosaurus; Britt et al., 2017, figures 4B, 23G; Wilson et al., 2011). Only the base of the neural spine is preserved in both vertebrae. It is around 1.5 times anteroposteriorly longer than lateromedially wide. In contrast to the cervical vertebrae, in this dorsal vertebra there is a well-marked PRSL (Figure 16C), as in many other sauropods (e.g., Shunosaurus, Mamenchisaurus, Moabosaurus, Europasaurus, Diplodocus; Britt et al., 2017; Carballido and Sander, 2014; Wilson, 1999). However, this lamina is absent in some sauropods (e.g., Tazoudasaurus, Patagosaurus, Spinophorosaurus, Mierasaurus; IVPP V9065; Allain and Aquesbi, 2008, table 2; PVL 4170; Remes et al., 2009; Royo Torres et al., 2017). The cross section of the neural spines reveals a thin septum separating both SDFs between them in dorsal view. Two long SPOLs are present: the LSPOLs that define a deep SPOF, and the MSPOLs within this fossa (Figure 16B). The MSPOLs are thinner and lower than the LSPOLs and are medial to them. The dorsal division of the SPOL is also seen in other sauropods, such as Spinophorosaurus (GCP-CV-4229) and Patagosaurus (PVL 4170). As in the anterior dorsal vertebrae, the SPDL is absent.

MPEF-PV 11000. This specimen is composed of an almost complete but highly deformed posterior dorsal vertebra (Figure 17). Whereas in mid-dorsal centra, the concave posterior articular face is deeper than the anterior, in posterior dorsal vertebrae the centrum has a flat anterior articular surface and slightly concave posterior one. This feature is similar to that observed in some basal sauropods (e.g., Tazoudasaurus, Amygdalodon, Volkheimeria; Allain and Aquesbi, 2008; Rauhut, 2003; PVL 4077) and more derived taxa (e.g., Shunosaurus, Omeisaurus, Spinophorosaurus, Patagosaurus, Jobaria, Europasaurus; Zhang, 1988, figures 24-28; He et al., 1988, figures 1923; Remes et al., 2009; Bonaparte, 1986; Sereno et al., 1999; Carballido and Sander, 2014).

The centrum of this vertebra is almost as dorsoventrally high as anteroposteriorly long (El: 0.9; see Table 4 in Appendix 1). The ventral surface is convex in cross section and lacks a ventral keel (Figure 17E). The lateral fossa has only the ventral edge well-marked and becomes shallower dorsally and posteriorly (Figure 17A, C), as in other sauropods (e.g., Tazoudasaurus, Mamenchisaurus; Allain and Aquesbi, 2008, figure 14E-F; Ouyang and Ye, 2002, figures 22-27). This fossa is not considered a true pleurocoel, that is, a fossa with all its margins as observed in other sauropods (e.g., Omeisaurus, Moabosaurus; He et al., 1988, figure 20; Britt et al., 2017, figures 3-4). In Patagosaurus, the pleurocoels are present throughout the dorsal series but gradually become an oval depression in posterior elements (Holwerda et al., 2021), which is similar to that observed in MPEF-PV 11000 (PVL 4170), although in some vertebrae of Patagosaurus this lateral fossa is deeper and has all of its margins distinct, with the dorsal one most pronounced (Holwerda et al., 2021, figures 11-17; $\mathrm{MACN}-\mathrm{CH}$ 936). The neural arch is dorsoventrally crushed and inclined to the left. Only a portion of the right parapophysis is preserved. The ACPL is thin and vertical, extending from the parapophysis to the anterodorsal margin of the centrum and reaching its anterior articular surface. The PRPL is as thin as the ACPL, and also has a vertical orientation in lateral view, although it is somewhat longer than it. The PPDL is clearly visible on both sides, having different degrees of inclination due to the general deformation of the vertebra (Figure $17 \mathrm{~A}, \mathrm{C})$. The prezygapophyses are flat and inclined ventromedially at an angle of 45 degrees in anterior view. As in all sauropods, this posterior vertebra lacks the TPRL due to the presence of a well-developed hypantrum with the same morphology as that present in MPEF-PV 11027 (Figure 17B). The CPRF is deep, as in Patagosaurus (Holwerda et al., 2021, figure 13). The PRCDF has the shape of an inverted triangle with its base oriented dorsally. The diapophysis is anteroposteriorly long and triangular in shape. The posterior surface of the distal end of the diapophysis lacks the excavation described for the posterior dorsal vertebrae of Patagosaurus (Holwerda et al., 2021). The PCDL runs from the ventral surface of the transverse process posteroventrally at an angle of 30 degrees, and encloses a deep and triangular CDF with the PPDL (Figure 17C). The postzygapophyses have a flat articular surface and do not have epipophyses. The hyposphene is a small rhomboid structure hanging over the neural canal (Figure 17D).

The neural spine is crushed dorsoventrally and displaced posterolaterally to the left side. Dis- 


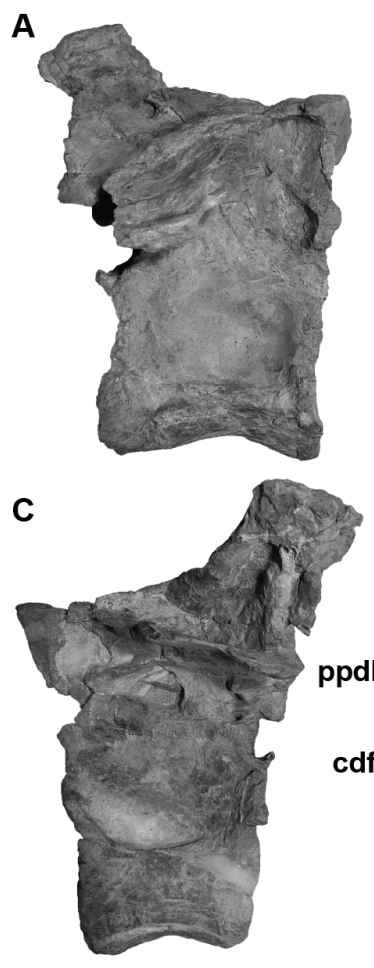

E

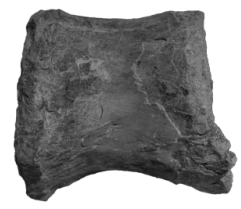

spol<smiles>CC1([SiH3])CCCCC1</smiles>

sprl

B
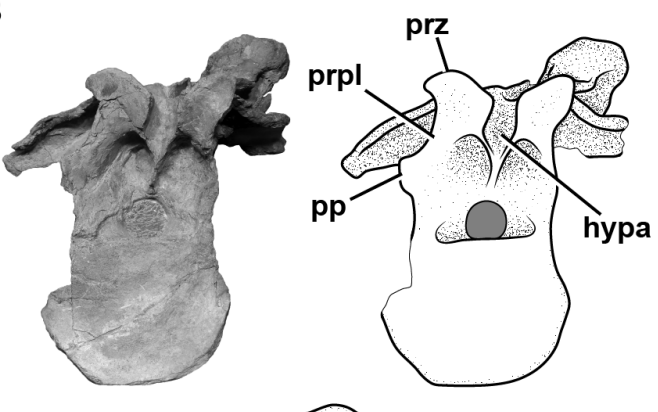

D
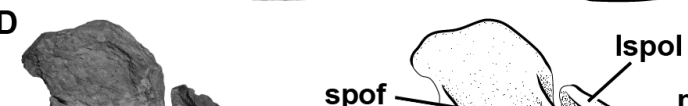

ppdl
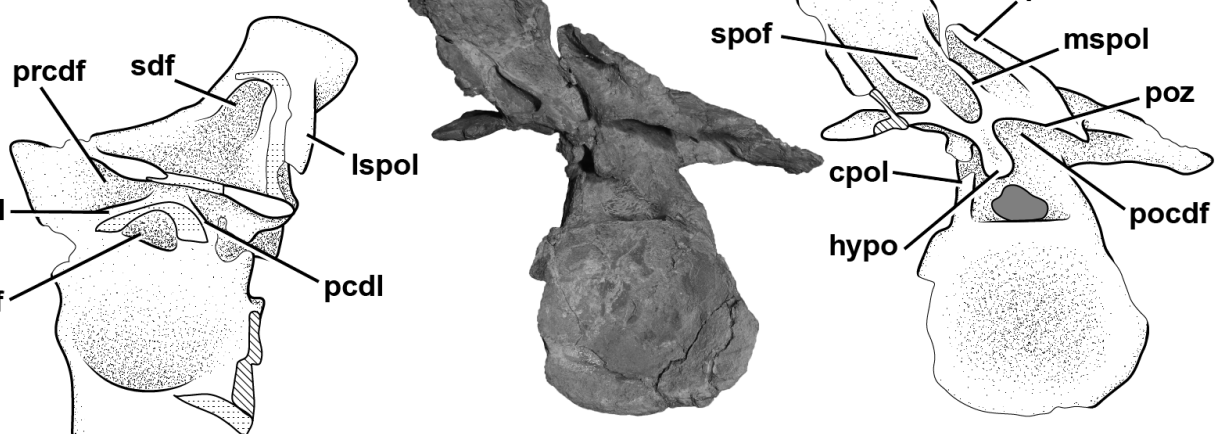

$\mathbf{F}$
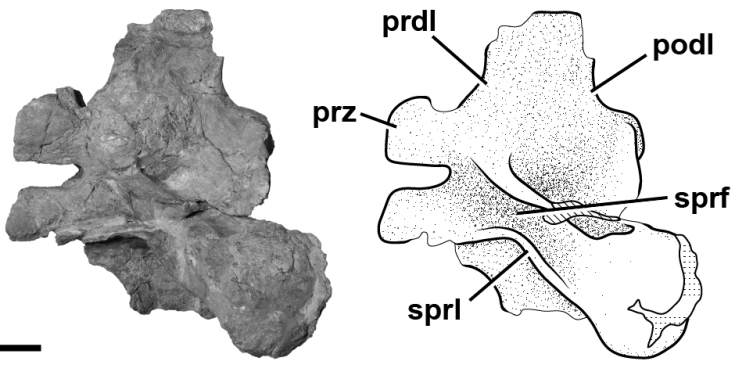

FIGURE 17. Posterior dorsal vertebra of Bagualia alba (MPEF-PV 11000) in right lateral (A), anterior (B), left lateral (C), posterior (D), ventral (E), and dorsal (F) views. Abbreviations: cdf, centrodiapophyseal fossa; cpol, centropostzygapophyseal lamina; dp, diapophysis; hypa, hypantrum; hypo, hyposphene; Ispol, lateral spinopostzygapophyseal lamina; mspol, medial spinopostzygapophyseal lamina; pcdl, posterior centrodiapophyseal lamina; pocdf, postzygapophyseal centrodiapophyseal fossa; podl, postzygodiapophyseal lamina; poz, postzygapophysis; pp, parapophysis; ppdl, paradiapophyseal lamina; prcdf, prezygapophyseal centrodiapophyseal fossa; prdl, prezygodiapophyseal lamina; prpl, prezygoparapophyseal lamina; prz, prezygapophysis; sdf, spinodiapophyseal fossa; spof, spinopostzygapophyseal fossa; sprf, spinoprezygapophyseal fossa; sprl, spinoprezygapophyseal lamina. Hatched pattern represents broken surfaces and dotted pattern represents putty. Scale bar equals $10 \mathrm{~cm}$.

tally the neural spine widens lateromedially, being 1.27 times lateromedially wider than anteroposteriorly long (Figure 17F). This is unlike Volkheimeria, in which the neural spine is around two times anteroposteriorly longer than lateromedially wide (Bonaparte, 1986, figure 67). The maximum lateromedial expansion of the neural spine in MPEF-PV 11000 is close to the distal tip, forming a slightly expanded, convexlateral margin. Such an expansion clearly contrasts with the triangular and more expanded aliform process present in several sauropods (e.g., Patagosaurus, Spinophorosaurus, Losillasaurus, Moabosaurus; MACN-CH 936; GCP-CV-4229; Royo Torres et al., 2021, figure 7;
Britt et al., 2017, figure 4). The morphology of Bagualia could be considered as a very incipient expansion, homologous to the triangular expansion in the aforementioned taxa, but was treated as an ambiguous character for purposes of phylogenetic analysis. Given the degree of variation observed in the development of this process in Patagosaurus, new materials from Bagualia could clarify the homology of this structure.

The SDF extends from the dorsal base of the transverse process to two thirds of the dorsoventral length of the neural spine (Figure 17A). This fossa is deep and has all its margins well-marked, as in sauropods such as Tazoudasaurus (Allain and 


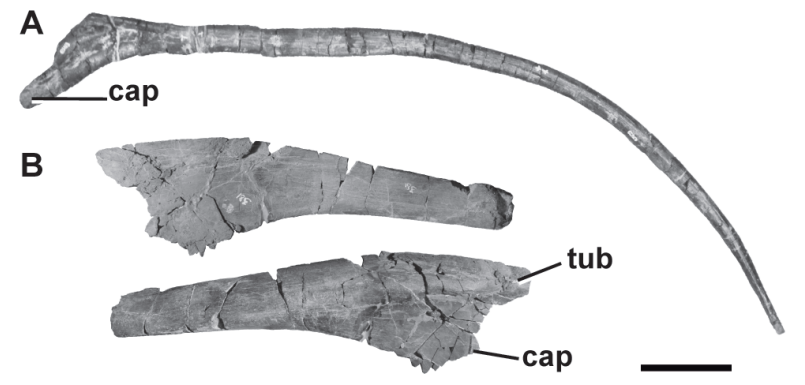

FIGURE 18. Dorsal ribs of Bagualia alba. A, MPEF-PV 11052 in posterior view; B, MPEF-PV 11053 in anterior and posterior views. Abbreviations: cap, capitulum; tub, tuberculum. Scale bar equals $10 \mathrm{~cm}$.

Aquesbi, 2008, figure 13) and Patagosaurus (MACN-CH 936; Holwerda et al., 2021, figure 13). In contrast, Volkheimeria does not have a marked SDF in its posterior dorsal vertebrae (Bonaparte, 1986, figures 66-67). As in the previous vertebrae, there are two (left and right) long LSPOLs that run from the dorsal surface of the postzygapophyses to the distal posterior tip of the neural spine (Figure 17D), and two MSPOLs that run medially to the LSPOLs and extend vertically from the posteromedial margin of the postzygapophyses to the dorsoventral mid-length of the neural spine. There is no PODL+LSPOL complex, which is present in Patagosaurus and Cetiosaurus (Holwerda et al., 2021). There are no signs of a PRSL or POSL, which are present in Volkheimeria (Bonaparte, 1986, figures 66-67). However, the anterior and posterior surfaces of the neural spine do feature a slight roughness.

\section{Dorsal Ribs}

The dorsal ribs recovered of Bagualia are represented by several isolated and mixed fragments that, as for the cervical ribs, cannot be assigned precisely to any of the described dorsal vertebrae. This description is mainly based on the two best preserved dorsal ribs (Figure 18). The right rib MPEF-PV 11052 is one of the anteriormost dorsal ribs of Bagualia and only lacks its capitulum and the proximal tip of the tuberculum (Figure 18A). The tuberculum is very long, is aligned with the capitulum, and the angle between the tuberculum and the shaft is approximately 45 degrees. This process is around two times anteroposteriorly longer than lateromedially wide. The shaft is $90 \mathrm{~cm}$ long, is triangular in cross section near the proximal part, and becomes rounded distally. The very long shaft and its alignment with the capitulum and tuberculum indicates that MPEF-PV 11052 had a

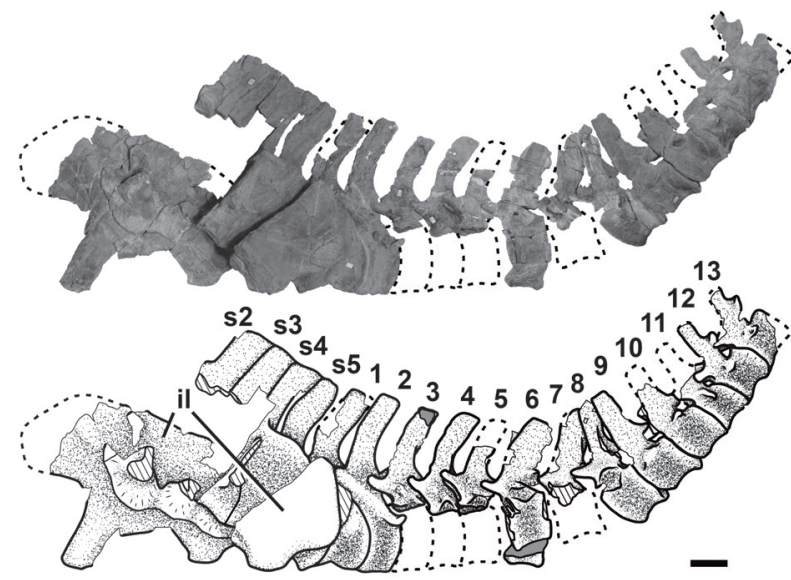

FIGURE 19. Articulated sacrum, ilia, and caudal vertebrae from first to tenth (MPEF-PV 11011), and possible eleventh, twelfth and thirteenth caudal vertebrae (MPEFPV 11044) of Bagualia alba. Abbreviations: il,ilium; s2s5, sacral vertebrae from second to fifth; 1-13, caudal vertebrae from first to thirteenth. Bold numbers indicate specimen MPEF-PV 11044. Dashed line represents reconstructed parts, hatched pattern for broken surfaces, and grey for sediment. Scale bar equals $10 \mathrm{~cm}$.

lateroventral orientation when articulating with a vertebra, so it is interpreted as a dorsal rib. In addition, the very long tuberculum and the circular shaft in cross section indicate that this rib could be the most anterior (maybe the first) of the dorsal series.

MPEF-PV 11053 is a left dorsal rib from a middle position in the dorsal series (Figure 18B). This rib lacks the capitulum and tuberculum and the distal half of the shaft. The proximal portion is anteroposteriorly flat. The shaft is directed vertically downward and curves slightly ventrally. This shaft is triangular in cross section near the proximal portion, and becomes plate-like with the major axis vertically oriented towards the most distal zone preserved. As in the cervical ribs, no pneumatophore or internal pneumaticity is present in the dorsal ribs of Bagualia, differing from derived titanosauriformes (Wilson and Sereno, 1998; Taylor et al., 2011; Carballido and Sander, 2014).

\section{Sacrum}

Only one incomplete sacrum was recovered from the Bagual locality (MPEF-PV 11011). This sacrum was found in natural articulation with both incomplete ilia and the first 10 caudal vertebrae (Figure 19). This specimen also comprises two posterior dorsal neural arches (see above) and one right pubis. In general, the pelvic girdle is crushed lateromedially and displaced anteroposteriorly, so the left elements are posteriorly positioned with 
respect to the right ones. The description of osteological units of the sacrum is made following the nomenclature of Wilson et al. (2011). Following this nomenclature, the vertebrae present parapophyseal and diapophyseal articulations on which the capitulum and tuberculum of the sacral rib become fused. Distally, the sacral ribs end in an acetabular arm (ventrally) and an alar arm (dorsally), which contacts the ilium.

Although the sacrum is incomplete, the preserved parts reflect that it was composed of five vertebrae (Figure 20A), as in Patagosaurus and more derived sauropods (Wilson, 2011). The first three sacral vertebrae are incomplete, almost completely lost, whereas the fourth and fifth are almost complete. The centrum is preserved in the posteriormost three sacral vertebrae (3-5), although the third sacral preserves only the posterior half (Figure 20B).

Despite not having all sacral vertebrae of Bagualia preserved, we have attempted to identify them according to the criteria proposed by Nesbitt (2011) to determine the primordial sacral vertebrae. When the primordial vertebrae are identified, it is easy to know if there were additions on the anterior, middle, or posterior zones of the sacral series.

The first sacral vertebra (S1, dorsosacral) only preserves a small portion of the acetabular arm of its sacral rib fused to the right ilium (Figure 20C) by a small and rugose contact with the second sacral rib. If it was complete, $\mathrm{S} 1$ would be fused close to the preacetabular process and the anterior margin of the base of the pubic peduncle, as in other sauropodomorphs (e.g., Xingxiulong, Leonerasaurus, Patagosaurus; Wang et al., 2017; Pol et al., 2011; PVL 4170).

The second sacral vertebra (S2, first primordial) consists of an incomplete sacral rib and most of its neural spine (Figure 20B-C). Its development and location are coincident with the definition of a first primordial sacral vertebra (Nesbitt, 2011). The sacral rib is composed of an almost complete acetabular arm and by an incomplete vertical alar arm, which are fused to the ilium. The acetabulum arm of the rib is located between the base of the pubic peduncle and the acetabulum, occupying around $60 \%$ of its anteroposterior length. The acetabular arm is circular in section, and its inferior portion defines a concave anterior surface. The area of attachment of the acetabular arm with the ilium is oval and expanded distally, with a rough surface of scratches in a radial orientation from the rib-ilium contact. This rib is far from the acetabular margin.
Judging from the shaft of the acetabular arm, this rib had a full lateral orientation.

The third sacral vertebra has preserved the posterior half of the centrum, sacral ribs, and most of the neural spine (Figure 20B-C). When complete, this vertebra would be located at the level of the posterior margin of the acetabulum and the anterior half of the ischial peduncle of the ilium. The centrum is subcircular in cross section and lacks pleurocoels or deep lateral fossae. On the left side of the centrum, there is a proximal portion of the sacral rib. The acetabular arm is broad and rounded in cross section, whereas the alar arm is laminar and dorsally elongated. The acetabular arm occupies the dorsal mid-height of the centrum and is continuous with the laminar and elongated alar arm, so the transverse foramen is not present in this element. The right sacral rib only preserves its ventral distal portion (acetabular arm), which is fused to the ilium and the second and fourth sacral ribs. The contact area between this rib and the fourth is slightly larger than that with the second. The acetabular arm of this rib is located at the level of the anterior margin of the ischial pedicel of the ilium, far from the acetabular margin. As in the previous rib, it is distally expanded and has several radial scratches on its surface. This acetabular arm is smaller than the previous one and has a posterodorsal orientation. Taking into account both proximal and distal portions of the ribs of this vertebra, we can infer that these were anterolaterally oriented before contacting the ilium.

The fourth sacral vertebra (second primordial) is nearly complete (Figure 20B-C). The centrum is around 1.5 times dorsoventrally taller than anteroposteriorly long and lacks any lateral fossa or pleurocoel. The left acetabular arm is robust and is attached to the centrum on the dorsoventral and anteroposterior mid-length of the centrum. This area of attachment of the rib occupies almost the entire anteroposterior length of the centrum. The transverse process is fused to the alar arm of the rib, forming a single and laminar complex that contacts the ilium and in this case is crushed, so it obscured part of the following vertebra. The complete rib attachment would be C-shaped (if not crushed), with the anterior surface concave (Figure 20B). No transverse foramen is present in this vertebra. The right sacral rib is fused to the ilium by a distally expanded acetabular arm, which is smaller than that of the previous rib. This rib is at the level of the posterior half of the ischial peduncle and the proximal portion of the postacetabular process. The degree of anterior orientation of the ribs of this 

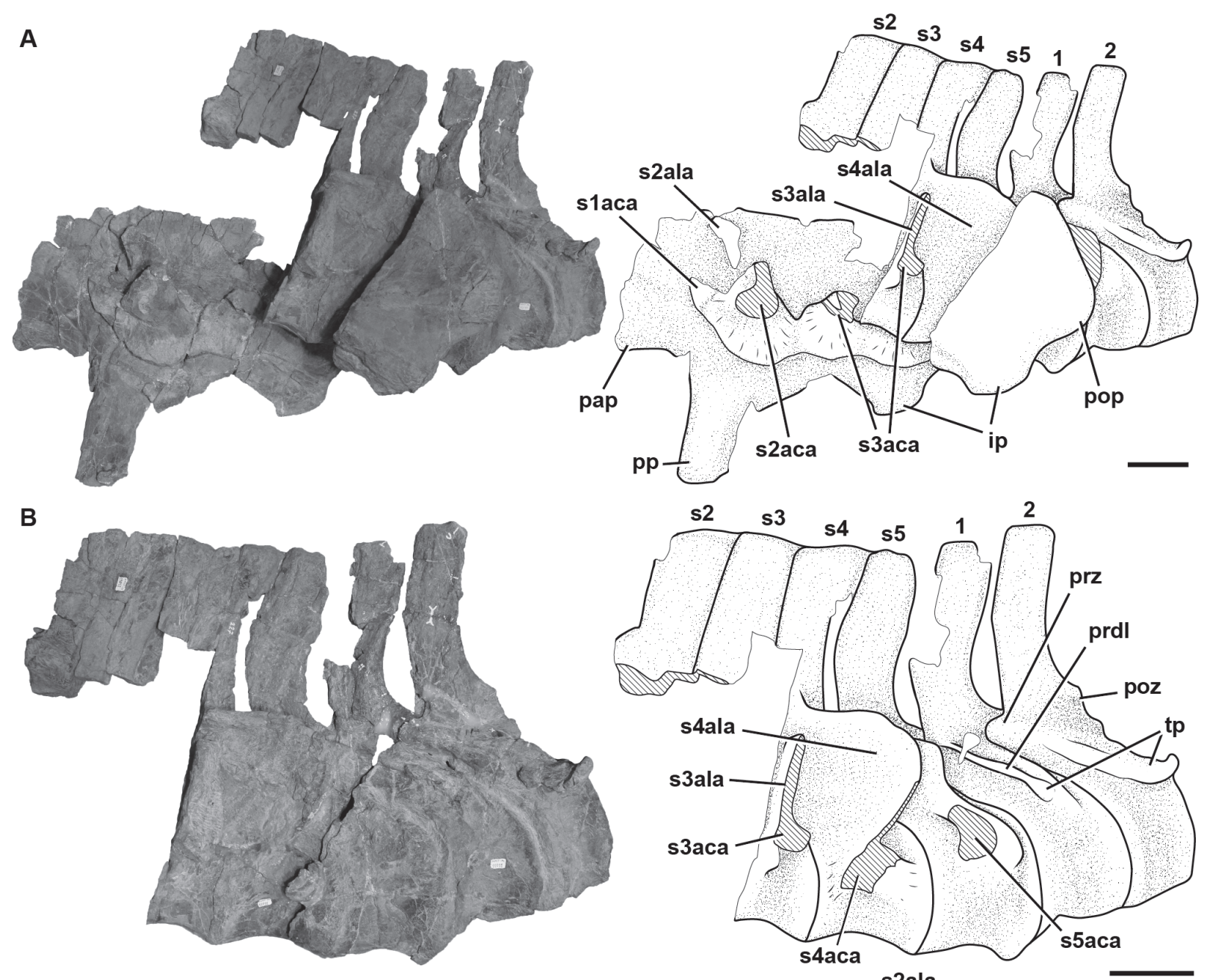

C
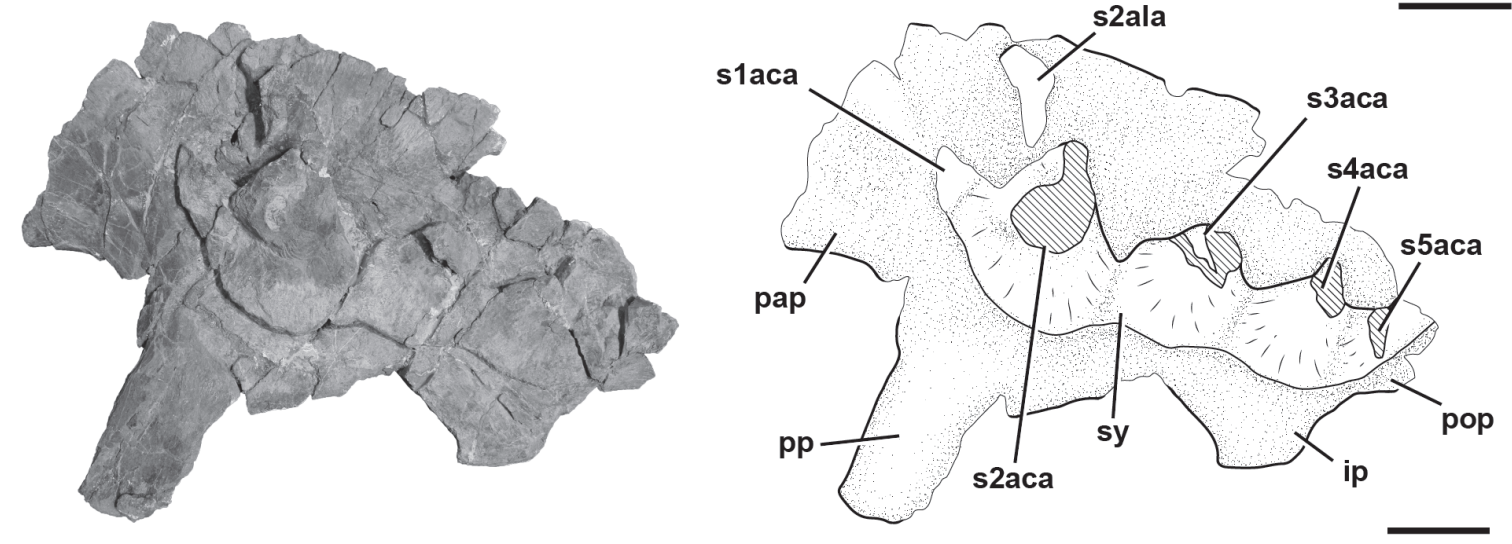

FIGURE 20. Articulated sacrum and first caudal vertebrae of Bagualia alba (MPEF-PV 11011). A, articulated ilia, sacral and first caudal vertebrae in left lateral view; B, sacral and first caudal vertebrae in left lateral view; C, right ilium in medial view. Abbreviations: aca, acetabular arm of the rib; ala, alar arm of the rib; ip, ischial peduncle; ns, neural spine; p, protuberance; pap, preacetabular process; pop, postacetabular process; poz, postzygapophysis; pp, pubic peduncle; prdl, prezygodiapophyseal lamina; prz, prezygapophysis; s1-s5, sacral vertebrae from second to fifth; sy, sacricostal yoke; tp, transverse process; 1, first caudal vertebra; 2, second caudal vertebra. Hatched pattern represents broken surfaces. Scale bar equals $10 \mathrm{~cm}$. 
vertebra is slightly greater than that of the S3. These characteristics are typical of the second primordial rib (Nesbitt, 2011). In contrast, the third sacral rib is fused to the ilium anterior to the ischial peduncle, and this is not consistent with a primordial sacral rib, so we considerate that the third vertebra is an insertion between the first and second primordial sacral vertebrae.

The fifth sacral vertebra (S5, caudosacral) lacks part of its sacral ribs and an anterior portion of the neural spine (Figure 20B-C). The most posterior location (on the posterior end of the postacetabular process) and the lesser development of the fifth sacral rib allow identification of the fifth sacral vertebra as a caudosacral. As in the previous vertebrae, the centrum is taller than anteroposteriorly long and no pleurocoels are present. The centrum has similar measurements to that of the anterior vertebra, which is different from Spinophorosaurus, in which the centrum of the fifth vertebra is shorter than that of the fourth one (GCP-CV 4229/HB 27; see above). The proximal portion of the acetabular arm of the rib joins the centrum at a higher point than that on the fourth sacral vertebra, just above of the dorsoventral mid-length of the centrum (Figure 20B). The area of attachment of the rib in the centrum is slightly smaller than that previous vertebra, occupying around $75 \%$ of the anteroposterior length of the centrum. The transverse process extends to the base of the neural arch. The sacral rib is smaller than that of the anterior vertebra (Figure $20 \mathrm{~B}-\mathrm{C}$ ). The acetabular arm of the right sacral rib is fused to the ilium at the level of its postacetabular process, just posterior to the iliac peduncle. This rib is more poorly developed than that on the previous vertebrae, but the area of attachment with the anterior rib is more extensive than the rest. Judging by the orientation of portions of the acetabular arm with the vertebral centrum and ilium, the rib of this vertebra would have been much more anteriorly oriented then the preceding vertebrae.

All preserved centra are subcircular in cross section and 1.5 times taller than long (measured in the complete fourth and fifth centra), as in other derived sauropods (e.g., Patagosaurus, Haplocanthosaurus, Diplodocus; PVL 4170; Hatcher, 1903, figure 15; Osborn, 1904, figure 3). In Spinophorosaurus, the first four sacral vertebrae have the same ratio, but the fifth is shortened, being around twice as tall as long (GCP-CV 4229/HB27). Most basal sauropodomorphs have sacral centra longer than tall (e.g., Massospondylus, Jingshanosaurus, Adeopapposaurus, Leonerasaurus, Mussaurus;
Cooper, 1981, figure 12C; Zhang and Yang, 1995, figure 17; Martínez, 2009, figure 16A; Pol et al.,2011, figure 8E; Otero and Pol, 2013, figure $4 \mathrm{C})$, whereas in the basal sauropod Melanorosaurus (NM QR 1551), the centra are equally long as tall. The lack of deep fossae or pleurocoels on the lateral surfaces of preserved centra S3-S5 (Figure 20B) is also seen in basal sauropodomorphs (e.g., Jingshanosaurus, Leonerasaurus, Mussaurus; Zhang and Yang, 1995, figure 17; Pol et al., 2011, figure 8E; Otero and Pol, 2013, figure 4E) and some advanced sauropods (e.g., Cetiosaurus, Galvesaurus; Upchurch and Martin, 2002; Barco, 2009). Well-developed pleurocoels in at least the primordial and caudosacral vertebrae are present in some derived eusauropods (e.g., Mamenchisaurus, Jobaria, Camarasaurus, Europasaurus, Euhelopus, Diplodocus; Ouyang and Ye, 2002, figure 29A; MNN TIG 3-5; BYU 9047; Carballido and Sander, 2014, figure 25C; Wilson and Upchurch, 2009; Hatcher, 1901). Unlike Bagualia, in Patagosaurus the lateral surfaces of the centra have deep fossae, although they are not considered true pleurocoels (PVL 4170, MACN-CH 935/226). Similar fossae are also present in Spinophorosaurus, although these are deeper still than those of Patagosaurus and there seems to be a dorsal margin in the fourth vertebra that could be an early stage of a true pleurocoel (GCP-CV 4229/HB 26). The rib is fused on the dorsoventral mid-length of the centrum in the third and fourth sacral vertebrae, whereas in the fifth sacral it migrates slightly dorsally (see above). The zone where the sacral ribs attach to the centrum is variable along a single sacral series or between taxa (Upchurch et al., 2004). The fourth sacral rib of Bagualia shows greater dorsoventral development than that in the fifth, as in Spinophorosaurus (GCP-CV 4229/HB $26)$. This variation in the dorsoventral extent of the parapophysis-rib junction is also observed in other taxa. For example, basal sauropodomorphs have parapophyses that occupy a greater dorsoventral length of the centrum posteriorly in the series, which is also greater in the second primordial vertebra or third sacral vertebra of these taxa (e.g., Adeopapposaurus, Mussaurus, Leonerasaurus; Martínez, 2009; Otero and Pol, 2013, figure 4C; Pol et al., 2011, figure 8F).

Also, there is variation in the anteroposterior location and extent of the parapophysis-rib junction along the same sacral series. In Bagualia, the junction in the fourth and fifth sacral vertebra is located at the anteroposterior mid-length of the centrum and occupies around of $80 \%$ and $75 \%$ of it, respec- 
tively. Basal sauropodomorphs have the parapophysis-rib junction on the anterior half of the centrum in the dorsosacral and primordial vertebrae, occupying around $60 \%$ of the anteroposterior length of the centrum (e.g., Yizhousaurus, Adeopapposaurus; Zhang et al., 2018, figure 4D; Martínez, 2009, figure 16). In the case of Plateosaurus, which has a caudosacral plus the two primordial sacral vertebrae, the junction in the caudosacral is on the midlength of the centrum (Galton, 2000, figure 5C). When a fourth sacral vertebra is present, the caudosacral vertebra has its parapophysis in the anteroposterior mid-length of the centrum, and the two other sacral vertebrae maintain their parapophysis in the anterior half in most basal sauropodomorphs (e.g., Xingxiulong, Mussaurus, Melanorosaurus; Wang et al., 2017, figure 4B; Otero and Pol, 2013, figure 4C; NM QR 1551), and possibly in Leonerasaurus (Pol et al., 2011, figure $8 \mathrm{E})$. As in Bagualia, Spinophorosaurus has fourth and fifth sacral vertebrae with their parapophysisrib junctions close to the anteroposterior mid-length of the centrum, whereas in the first three sacral vertebrae of Spinophorosaurus they are restricted to the anterior half (GCP-CV 4229). The eusauropod Mamenchisaurus has the first four sacral vertebrae with the parapophysis-rib junction on the anterior half of the length of the centrum, whereas the fifth is close the mid-length (Ouyang and Ye, 2002, figure 29A), as in the diplodocid Apatosaurus (Gilmore, 1936, figure 7) and the macronarian Europasaurus (Carballido and Sander, 2014, figure 25A). In contrast, the neosauropod Haplocanthosaurus has all sacral ribs fused to the anterior half of the centrum (Hatcher, 1903, plate 5). This indicates that, despite the fact that the articulation between the parapophysis and the sacral rib is variable both within the same axial series and between taxa, in most taxa the rib attaches to the centrum on the mid-length on the caudosacral vertebra.

The sacral ribs are concave anteriorly and posteriorly, as in Spinophorosaurus (GCP-CV 4229). The shape in cross section of the ribs is variable along the same sacral series and between taxa (e.g., Pol et al., 2011; Otero and Pol, 2013; Martínez, 2009). Due to incompleteness of the material, it is not possible to observe the presence of an intracostal fenestra, which is present in other sauropods (e.g., Patagosaurus; MACN-CH 935). The expanded acetabular arms of the sacral ribs are fused to the right ilium and contact one another (except for the first sacral rib which is incomplete), forming a sacricostal yoke, as in the sau- ropodomorph Sarahsaurus (Marsh and Rowe, 2018) and derived sauropods (e.g., Wilson, 2002; Allain and Aquesbi, 2008). The sacral ribs of the S2 and S3 are farther from the edge of the acetabulum than those of Spinophorosaurus (GCP-CV 4229). Due to the great distance between the ribs and the dorsal margin of the acetabulum, the sacral vertebrae could not contribute to the dorsal margin of the acetabulum, which contrasts with the typical condition in eusauropods (Wilson and Sereno, 1998; Wilson, 2002). This exclusion of the sacral ribs from the acetabular margin is typical of non-sauropod sauropodomorphs (Marsh and Rowe, 2018).

Only the first sacral vertebra completely lacks its neural spine. All neural spines are plate like, longer than wide, and lack lateral fossae. Distally, the neural spines are not markedly widened and have their anterior, dorsal, and posterior margins flat in lateral view, as in most other sauropods (e.g., Shunosaurus, Cetiosaurus, Mamenchisaurus; IVPP V9065; Upchurch and Martin, 2002; Ouyang and Ye, 2002, figure 29). In contrast, some other taxa have a strong distal widening of their sacral neural spines (e.g., Moabosaurus, Camarasaurus; Britt et al., 2017, figure 6; BYU 9047). Unlike Bagualia, Patagosaurus has neural spines that are more distally widened and anteroposteriorly short, with all margins rounded and the dorsal surface convex (PVL 4170). In Bagualia, the neural spines lack deep lateral fossae and the saddleshaped distal end seen in Spinophorosaurus and Patagosaurus (GCP-CV 4229; Holwerda et al., 2021). The lateral surfaces of the sacral neural spines of Bagualia lack SPDLs, as in Patagosaurus (Holwerda et al., 2021). The SPDL in sacral vertebrae is present in some eusauropods (e.g., Spinophorosaurus, Moabosaurus; MACN-CH 935; Remes et al., 2009; Britt et al., 2017) and several neosauropods (e.g., Camarasaurus, Diplodocus, Europasaurus; BYU 9047; CM 94; Carballido and Sander, 2014). All sacral neural spines are fully fused and curved posteriorly from the first third of its dorsoventral height as seen in the last two vertebrae. The neural spines are around 1.8 times dorsoventrally longer than the anteroposterior length of the centrum, as in most sauropodomorphs except diplodocoids, in which the ratio is nearly 4 .

\section{Caudal Vertebrae}

A total of 45 caudal vertebrae assigned to Bagualia alba have been found in the Cañadón Bagual locality. A few elements are articulated 
(MPEF-PV 11011, MPEF-PV 11044, MPEF-PV 3316 ), but the majority were found isolated and treated as different specimens. Caudal vertebrae were divided into anterior, middle, and posterior caudal elements, using as reference the specimen MPEF-PV 11011 (which preserves the first 10 caudals) and the sequence changes in caudal morphology observed in other sauropods, such as Spinophorosaurus (GCP-CV-4229). The division of the caudal vertebrae in these three categories is based on anatomical differences such as: 1) the elongation index (in the case of both anterior and posterior faces being measurable, the average of both values was used; see Table 5 in the Appendix $1) ; 2$ ) the degree of inclination of the neural spine; and 3 ) the development of the transverse processes.

The anterior and middle section of the caudal sequence is represented by the specimen MPEFPV 11011 (Figure 19). This specimen is considered a late juvenile, as many of the caudal vertebrae lack their corresponding transverse processes, and the third, fourth, fifth, and seventh caudal vertebrae are solely represented by the neural arches, as their centra were not fused at the time of death.

Anterior caudal vertebrae. The centrum of the first two caudal vertebrae is at least twice as dorsoventrally higher as anteroposteriorly long (Figure 20B), as in all sauropods (Upchurch et al., 2004; Otero et al., 2015). The centra are amphicoelous, with concave anterior and posterior articular surfaces as in other sauropods (e.g., Patagosaurus, Haplocanthosaurus, Camarasaurus; PVL 4170; McIntosh and Williams, 1988, figure 10; McIntosh et al., 1996). This morphology differs from the slightly procoelous centra present in Losillasaurus (Royo Torres et al., 2021) and the strongly procoelous centra seen in mamenchisaurids and several turiasaurs (e.g., Mamenchisaurus, Mierasaurus, Moabosaurus; Ouyang and Ye, 2002, figures 3031; Royo Torres et al., 2017; Britt et al., 2017), most titanosaurs (e.g., Wilson, 2002; Upchurch et al., 2004), and diplodocoids (Whitlock, 2011). The ventral edges of the centra are concave in lateral view, and the ventral surface is convex in cross section. No articular facets for haemal arches are present on the first two caudal vertebrae of MPEPV 11011, or on other specimens interpreted as anteriormost caudals (e.g., MPEF-PV 3315, MPEF-PV 3402, MPEF-PV 3407). This matches the condition in other sauropods, in which the haemal arches appear around the third or fourth caudal vertebra. The ventral surface does not have a ventral keel, nor the deep, longitudinal groove described for other sauropods (e.g., Vulcanodon, Cetiosaurus, Patagosaurus, Camarasaurus, Haplocanthosaurus, Omeisaurus; Cooper, 1984; Holwerda et al., 2021; Upchurch and Martin, 2002; Upchurch et al., 2004; He et al., 1988) or the longitudinal ridges observed in the basal sauropodomorph Thecodontosaurus (Benton et al., 2010, figure 9G). The lateral surfaces of the centra lack a discrete fossa. The neural arch is located in the anterior half of the centrum, closer to the anterior articular face. In the first two caudal vertebrae, the neurocentral suture is not visible, and the transverse processes are, at least superficially, completely fused. The transverse processes are well developed and posterolaterally oriented, with a broad base. They are somewhat anteroposteriorly longer than dorsoventrally tall and are located on the centrum near its dorsal surface. The PRDL is anterodorsally oriented from the anterior margin of the transverse process towards the lateral surface of the prezygapophyseal processes, being similar in development and orientation to that observed in Tazoudasaurus (Allain and Aquesbi, 2008, figure 16A-B; Figure 20B) and other taxa. In the second caudal vertebra, the PRDL is shorter and thicker than in the first. The prezygapophyses of the first caudal vertebrae are anterodorsally oriented at an angle of 45 degrees. Postzygapophyses are short and laminar, and the hyposphene cannot be recognized. As in Patagosaurus (PVL4170), the pre- and postzygapophyses surpass the articular edges of the centrum. At base, the neural spines of the anteriormost caudals are vertically directed, whereas distally they bend slightly posteriorly as in other sauropodomorphs (e.g., Pulanesaura, Spinophorosaurus, Patagosaurus; McPhee et al., 2015, figure 3F; GCP-CV-4229-HB 28/29; MACN-CH 935-217). The height of the spines is slightly higher than their centra. The distal widening in the anteriormost caudal vertebrae of Bagualia is similar to that observed in some other sauropods (e.g., Shunosaurus, Patagosaurus, Omeisaurus; IVPP V 9065; MAC-CH 935; He et al., 1988, figures 33, 34A). In other taxa, however, this widening is better developed, and the dorsal margin is above the lateral margin, giving them a rhombus shape in anterior view (e.g., Spinophorosaurus, Camarasaurus; Remes et al., 2009, figure 3F; McIntosh et al., 1996, figures 43, 44). The dorsal margin of the neural spine in the anteriormost caudal vertebrae of Bagualia is straight, as in most sauropods (e.g., Antetonitrus, Shunosaurus, Camarasaurus; McPhee et al., 2014, figure 6B; IVPP V 9065; Mclntosh et al., 1996, figures 43,44$)$. The distal widening of the 
neural spine in the eusauropod Spinophorosaurus is greater than that observed in Bagualia (Remes et al., 2009, figure 3E-F). Also Spinophorosaurus has a saddle-shaped or concave dorsal margin in lateral view, which is also present in other eusauropods (e.g., Patagosaurus, Mamenchisaurus, Jobaria, Moabosaurus; Remes et al., 2009, figure 3E-F; Holwerda et al., 2021; Pi et al., 1996, figure 4; MNN TIG 3; Britt et al., 2017, figure 24A-H) and neosauropods (e.g., Haplocanthosaurus, CM 879). The eusauropod Tonganosaurus has a variant of this morphology, since within the concavity of the dorsal margin there is a central elevation in the anterior-middle caudal vertebrae (Li et al., 2010, figure $2 \mathrm{Q}-\mathrm{S}$ ). Two neural spine laminae are developed in the anteriormost caudals. The SPRLs and SPOLs are thin and reach the dorsoventral half of the neural spine, where they fade. All caudal vertebrae lack pre- or postspinal laminae, as in Patagosaurus (PVL 4170, MACN-CH 935) and Spinophorosaurus (GCP-CV-4229). The presence of prespinal laminae in anterior caudal vertebrae is common in derived eusauropods (Tschopp et al., 2015) such as Moabosaurus (Britt et al., 2017, figure 24) and Jobaria (Sereno et al., 1999), and most neosauropods.

The third, fourth, fifth, and seventh caudal vertebrae consist of isolated neural arches (Figures $21,22 D-F)$, whereas the sixth, eighth, ninth, and tenth are almost complete (Figures 22, 23A-C). The absence of fused centra or transverse processes between the third and eighth caudal vertebrae indicates that in this area of the tail, the junctions between the centrum and neural arch, and the centrum-neural arch to the transverse process, were weak or not fully closed. In contrast, in the ninth and tenth caudal vertebrae the suture is not visible. The lateral surfaces of the centrum have a shallow concavity, which is not bounded by marked margins, as also occurs in many sauropods (e.g., Tazoudasaurus, Patagosaurus; Allain and Aquesbi, 2008, figures 17-18; PVL 4170). As observed in the sixth and eighth caudal vertebrae, the transverse process articulates with the ventral section of the pedicels of the neural arch and the dorsal end of the centrum. In the neural arches, the articular surface for the transverse process is located at the anteroposterior mid-length and is triangular in shape, with the apex directed dorsally. The articular surface for the transverse process in the centrum (observed in the sixth and eighth caudal vertebrae) form an inverted triangle with its apex directed ventrally (Figure 22A, D).
In the third caudal vertebra, between the dorsal margins of the articular facet for the transverse process and the lateral surface of the prezygapophysis, there is a crest-like protuberance oriented anterodorsally (Figure 21A). This crest-like protuberance is in a similar position to the PRDL seen in the first two caudal elements. Unlike the PRDL in those vertebrae, however, in the third caudal this crest is shorter and becomes thick at its mid-length with a striated surface. Posteriorly, in the fourth and fifth caudal elements, this structure is like a rounded protuberance with a slightly striated surface and a more vertical orientation (Figure $21 \mathrm{~F})$. By the sixth vertebra, this protuberance is just a subtle elevated area with a smooth surface (Figure 22A). This protuberance is also observed in other specimens corresponding to the anterior and middle portion of the axial series (see below). In the rest of the vertebrae of MPEF-PV 11011, this protuberance is only observed as a small, rounded bump on the right lateral surface of the ninth caudal (Figure 23A). This feature has not been recognized in other sauropods (e.g., Cetiosaurus, Patagosaurus; Upchurch and Martin, 2002, figure7; PVL 4170). Towards the more posterior vertebrae of MPEF-PV 11011, the prezygapophyseal processes show a dorsoventral orientation at a lower angle than that observed in more anterior elements (around 30 degrees). In all anterior caudal vertebrae with preserved centra, the prezygapophyses extend beyond the anterior articular surface of the centrum. Postzygapophyses are laterally exposed in the third and eighth caudal vertebrae (Figures 21A, 22D). A laminar hyposphene (present in several sauropods; Upchurch et al., 2004 ) is not observed in any of the preserved caudal vertebrae of Bagualia. The neural spines are plate-like and around three times anteroposteriorly longer than mediolaterally wide. The total height of the neural spine is around 3.5 times its minimum anteroposterior length in the third caudal and decreases posteriorly, being 2.2 times by the eighth caudal vertebra. The SPOL and SPRL extend from the zygapophyses to the dorsoventral half of the neural spine (Figure 21B-C).

Middle caudal vertebrae. The centra of the middle caudal vertebrae are more elongate than those of the anterior caudals (between 1 and 2 times longer than tall; see Table 5 in Appendix 1), and the neural arch is located near the anterior articular surface (Figure 24). The articular faces are equally concave and lateral fossae are present (Figure 24A, E, I), unlike the anterior caudal vertebrae. The ventral surface is convex in cross section and the 
A
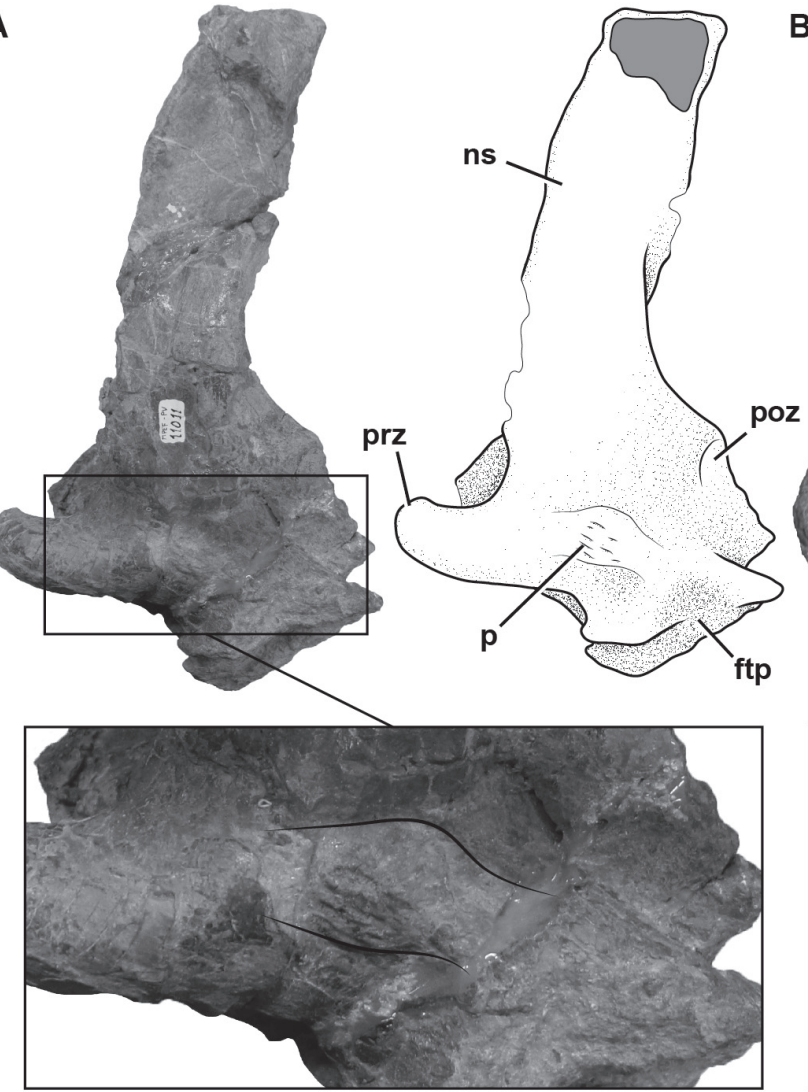

B

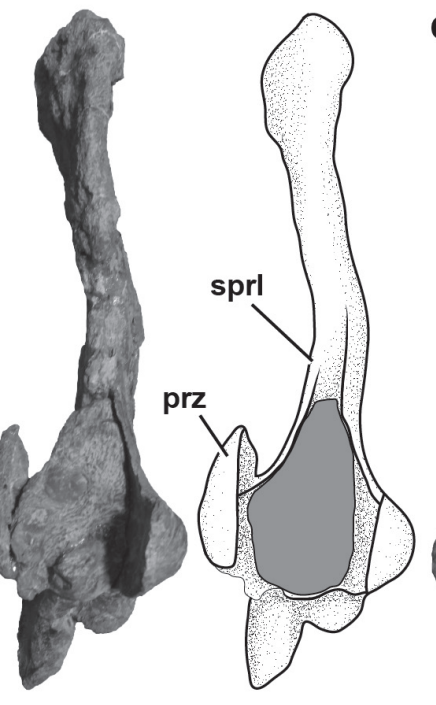

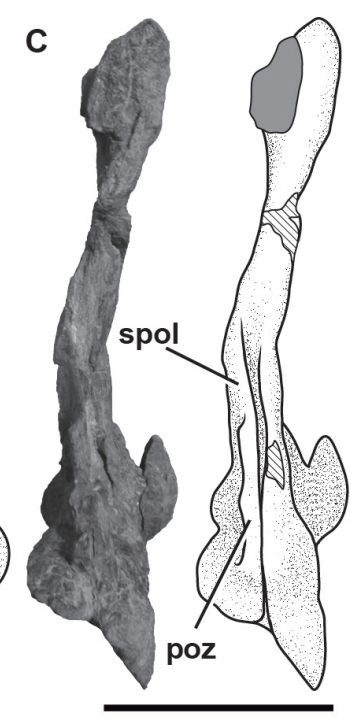
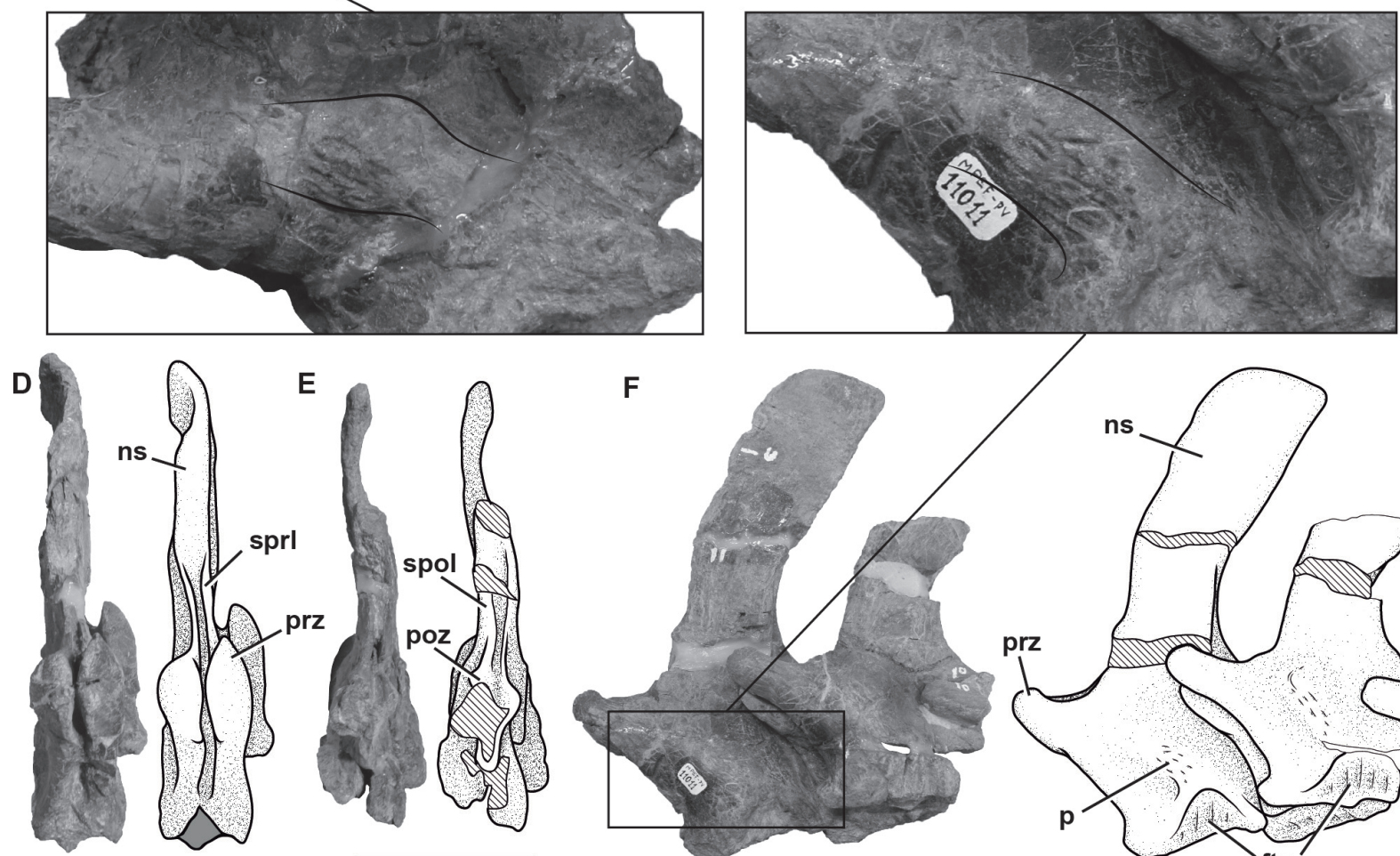

E
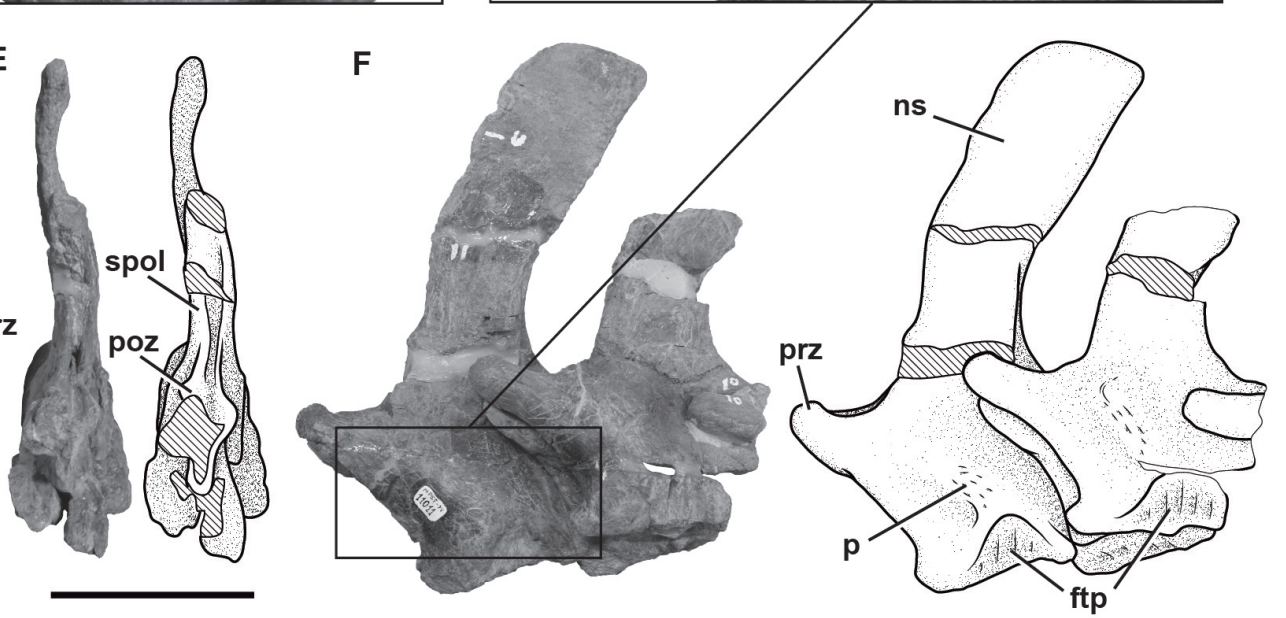

FIGURE 21. Anterior caudal neural arches of Bagualia alba (MPEF-PV 11011). A-C, third caudal neu-

ral arch in left lateral (A), anterior (B), and posterior (C) views. D-F, fourth and fifth caudal neural arches in anterior (D), posterior $(\mathbf{E})$, and left lateral $(\mathbf{F})$ views. Abbreviations: ftp, facet for transverse process; $\mathbf{n s , ~ n e u r a l ~ s p i n e ; ~} \mathbf{p}$, protuberance; poz, postzygapophysis; prz, prezygapophysis; sprl, spinoprezygapophyseal lamina; spol, spinopostzygapophyseal lamina. Hatched pattern represents broken surfaces and grey form represents sediment. Scale bar equals 10 $\mathrm{cm}$.

articular facets for haemal arches are well marked. The transverse processes are dorsoventrally flat and laterally projected and are located on the dorsal margin of the centrum (Figure 24A, C). As expected, development of the transverse processes decreases throughout the caudal series, and the posteriormost middle caudal vertebrae exhibit a short, laminar process (Figure 24E, I). The bump-like process above the transverse processes described in the anterior caudal vertebrae is present in the middle elements of the tail, although here this structure becomes even lower 

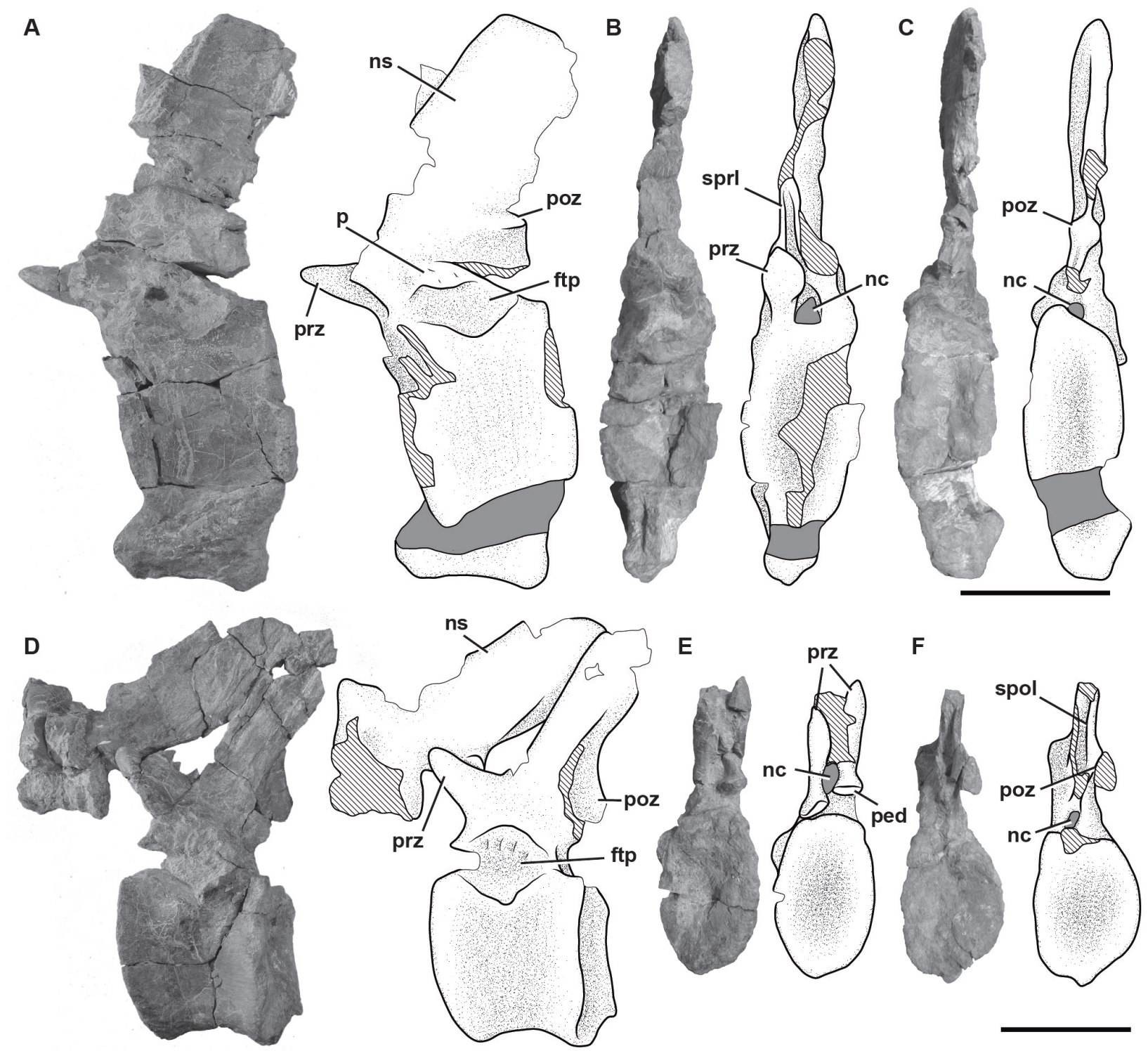

FIGURE 22. Sixth, seventh, and eighth caudal vertebra of Bagualia alba (MPEF-PV 11011). A-C, sixth caudal vertebra in left lateral (A), anterior (B), and posterior (C) views. D-F, seventh and eighth caudal vertebrae in left lateral (D), anterior (E), and posterior (F) views. Abbreviations: ftp, facet for transverse process; nc, neural canal; ns, neural spine; p, protuberance; ped, pedicel; poz, postzygapophysis; prz, prezygapophysis; spol, spinopostzygapophyseal lamina; sprl, spinoprezygapophyseal lamina. Hatched pattern represents broken surfaces and grey form represents sediment. Scale bar equals $10 \mathrm{~cm}$.

(Figure 24A, E, I). The prezygapophyseal processes are well developed, directed anteriorly at an angle less than 30 degrees, and surpass the anterior face of the centrum. The pre- and postzygapophyses are almost vertical, and no laminar hyposphene is present. The postzygapophyses surpass the posterior face of the centrum due to a more posterior inclination of the neural spine in these vertebrae. The neural spines maintain consistent anteroposterior length and lateromedial width throughout their dorsoventral height. The total height of the neural spine is less than two times its minimum anteroposterior length. They are posterodorsally projected, ranging from an angle of around 80 degrees in anterior middle caudals to a more posterior inclination (around 60 degrees) in the posteriormost middle caudal vertebrae. The SPRLs and SPOLs are shorter than in anterior caudal vertebrae and do not extend beyond the beginning of the neural spine.

Posterior caudal vertebrae. Posterior caudal vertebrae are recognized as those which centra are 

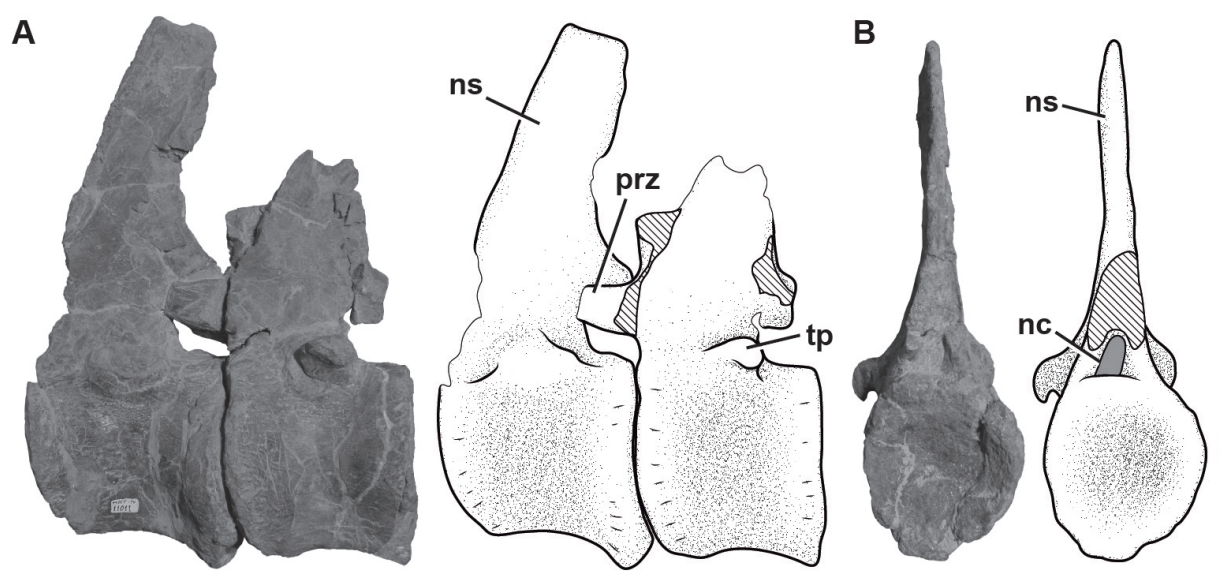

C
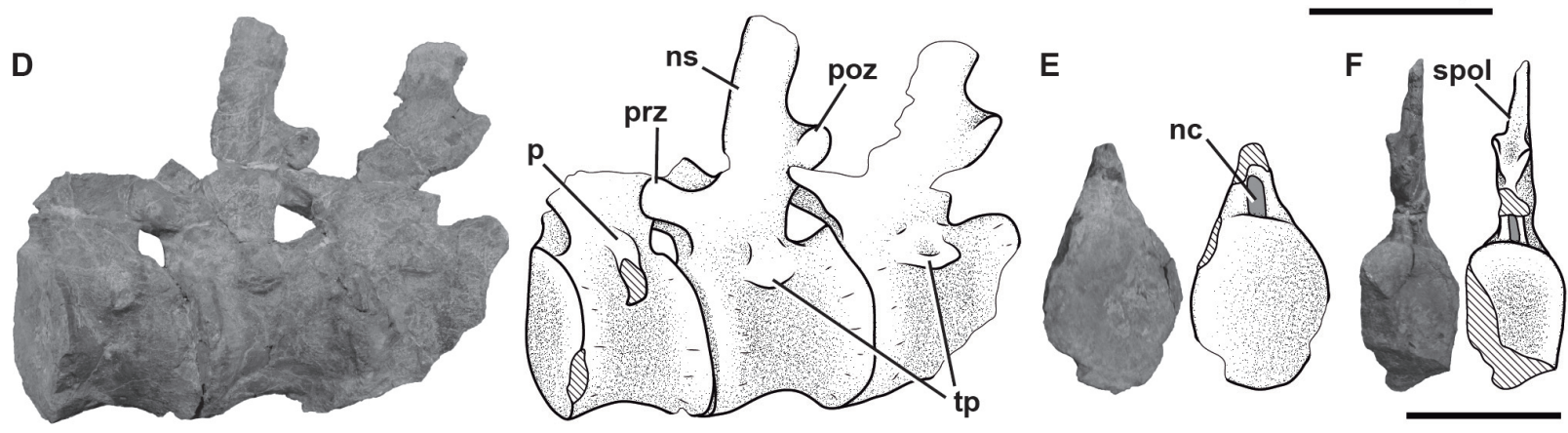

FIGURE 23. Ninth and tenth, and possible eleventh, twelfth and thirteenth caudal vertebrae of Bagualia alba. A-C, ninth and tenth caudal vertebrae (MPEF-PV 11011) in left lateral (A), anterior (B), and posterior (C) views. D-F, possible eleventh, twelfth and thirteenth caudal vertebrae (MPEF-PV 11044) in left lateral (D), anterior (E), and posterior (F) views. Abbreviations: nc, neural canal, ns, neural spine; p, protuberance; poz, postzygapophysis; prz, prezygapophysis; spol, spinopostzygapophyseal lamina; tp, transverse process. Hatched pattern represents broken surfaces and grey form represents sediment. Scale bar equals $10 \mathrm{~cm}$.

more than twice as long as tall (see Table 5 in the Appendix 1), transverse processes are completely missing, and no lateral fossae are present (Figure 25A, E, I). Preserved posterior caudal centra have concave anterior and posterior articular surfaces, as in basal sauropods (e.g., Upchurch et al., 2004), with a few exceptions (e.g., Mamenchisaurus; Ouyang and Ye, 2002). The protuberance observed above the transverse process in the anterior and middle caudal vertebrae is not present in any of the posterior caudals (Figure 25). The facets for the haemal arches are still present in some posterior caudals, developed to a greater degree than in the anterior caudals (MPEF-PV 3319/3322). The anterior margin of the neural arch is straight. The prezygapophyses are slightly marked and ovoid in shape. They do not surpass the anterior articular surface of the centra. On the anteriormost posterior caudal vertebrae, the postzygapophyses are visible as low surfaces at the base of the neural spine (Figure 25A, D), whereas in more posterior vertebrae they cannot be recognized (Figure 25E, H, I, $\mathrm{L})$. Throughout the posterior caudal vertebrae, the angle of the neural spine continues to decrease, varying from about 40 degrees (Figure 25A) to less than 10 degrees in the most posterior caudal vertebra preserved (Figure 25I). The centrum of the posteriormost caudal vertebra preserved is rod-like with expanded articular ends (Figure 25K).

\section{Transverse Processes}

Five isolated caudal transverse processes have been found, four of which are right ones (MPEF-PV 11001; MPEF-PV 11002; MPEF-PV 11003; MPEF-PV 11004) and one left (MPEF-PV 11005). These have the same morphology: dorsoventrally flattened with straight anterior and concave posterior margins in dorsal view. The distal dorsal surface bears an anteroposteriorly directed step with roughened surface; the ventral surface is smooth.

\section{Haemal Arches}

A total of 13 haemal arches were found isolated or in association with some of the caudal vertebrae preserved (Figure 26). As in basal 


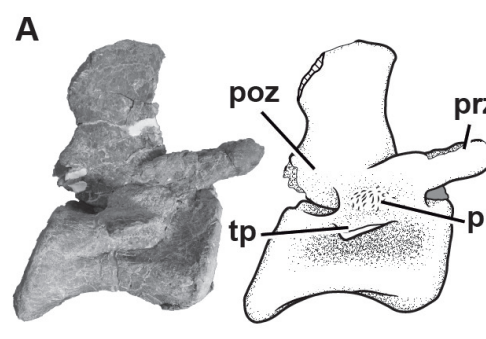

E

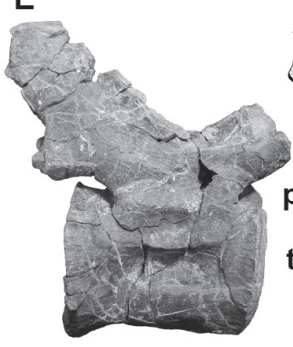

I

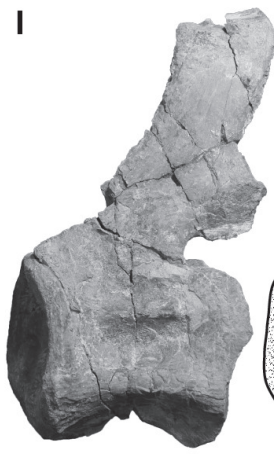

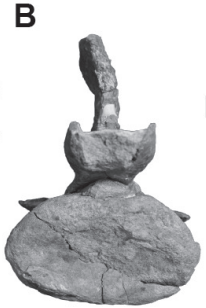
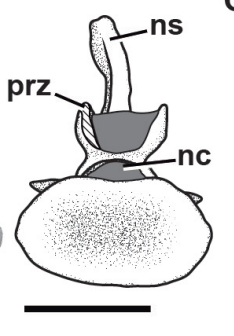

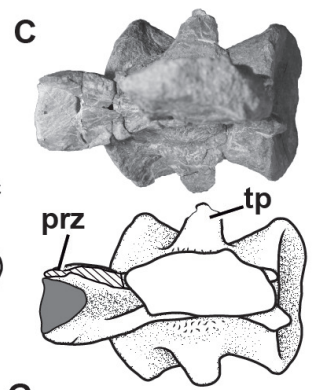

$\circ$
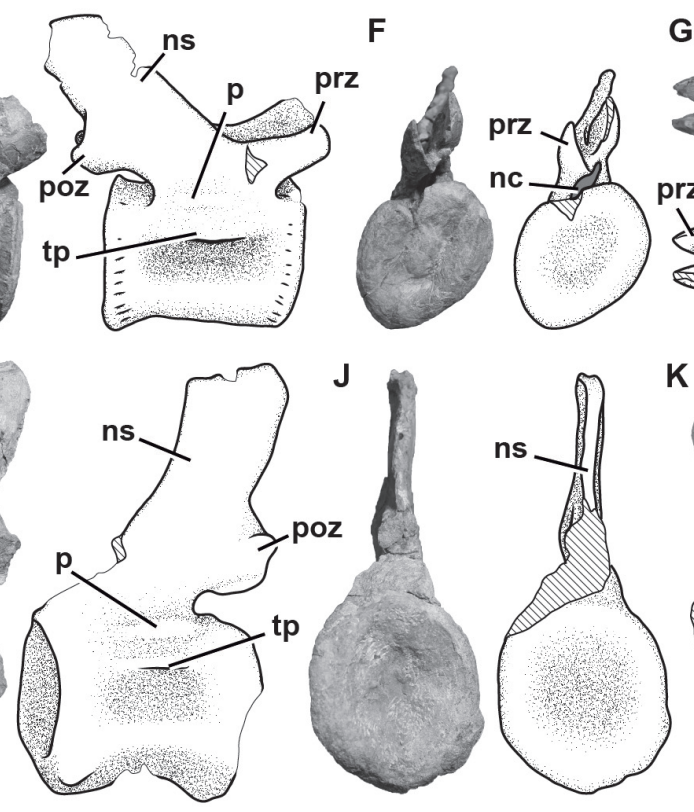

$\mathrm{J}$

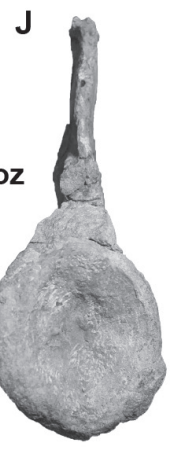

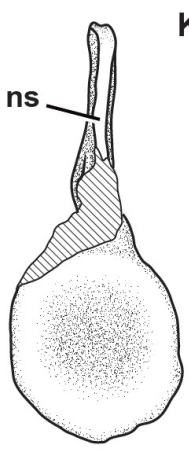

K

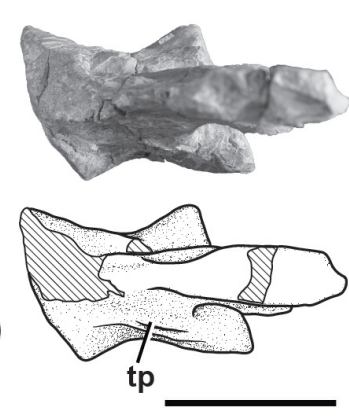

D

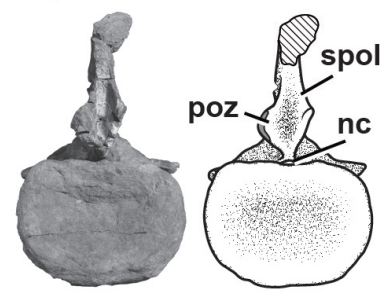

$\mathrm{H}$

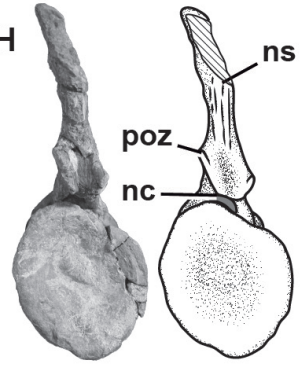

L

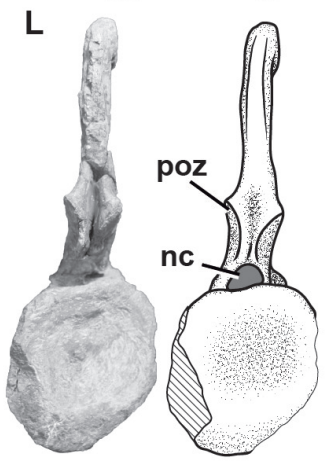

FIGURE 24. Middle caudal vertebrae of Bagualia alba. A-D, caudal vertebra MPEF-PV 3300 in right lateral (A), anterior (B), dorsal (C), and posterior (D) views. E-H, caudal vertebra MPEF-PV 3324 in right lateral (E), anterior (F), dorsal (G), and posterior (H) views. I-L, caudal vertebra MPEF-PV 3346 in left lateral (I), anterior (J), dorsal (K), and posterior (L) views. Abbreviations: nc, neural canal; ns, neural spine; p, protuberance; poz, postzygapophysis; prz, prezygapophysis; tp, transverse process. Hatched pattern represents broken surfaces and grey form represents sediment. Scale bar equals $10 \mathrm{~cm}$.

sauropodomorphs, the haemal arches are forked proximally, producing the characteristic Y-shape (Otero et al., 2012). All haemal arches have proximally expanded arms bridged by the intercentrum, as in most basal sauropods (e.g., Tazoudasaurus, Patagosaurus, Omeisaurus, Zby; Allain and Aquesbi, 2008; Bonaparte, 1986, figure 48; He et al., 1988, figure 39; Mateus et al., 2014, figure 2E) and flagellicaudatans (e.g., Wilson, 2002). This condition contrasts with that of Losillasaurus and some basal neosauropods, which have anterior and middle haemal arches bridged proximally, but their posterior ones open dorsally (Royo Torres et al., 2021). Having unbridged haemal arches throughout the caudal series is a derived condition present in some basal eusauropods (e.g., Shunosaurus, Mierasaurus; Zhang, 1988, figure 40; Royo Torres et al., 2017), rebbachisaurids, and macro- narians (Wilson, 2002; Upchurch et al., 2004). The complete anterior arches (Figure 26A, C, E) have a haemal canal that occupied around $20 \%$ of the total chevron length, which increases Table 6 in the Appendix 1). This ratio is similar to that of the anterior haemal arches of some other eusauropod taxa, such as Spinophorosaurus (18-23\%; GCP-CV 4229), but greater than that of others (e.g., Patagosaurus: 9-11\%, Mamenchisaurus: 9-10\%; MACN$\mathrm{CH}$ 935; Ouyang and Ye, 2002, plate XV). However, the extension of the haemal canal of Bagualia is lower than that of some turiasaurs (e.g., Losillasaurus: $27 \%$, Narindasaurus: $55.3 \%$, Mierasaurus: 41\%; Royo Torres et al., 2021; MNHN MAJ 425; Royo Torres et al., 2017) and most titanosauriforms (Curry Rogers and Foster, 2001; Wilson, 2002). All haemal arches have concave proximal anterior and posterior facets, with the posterior 

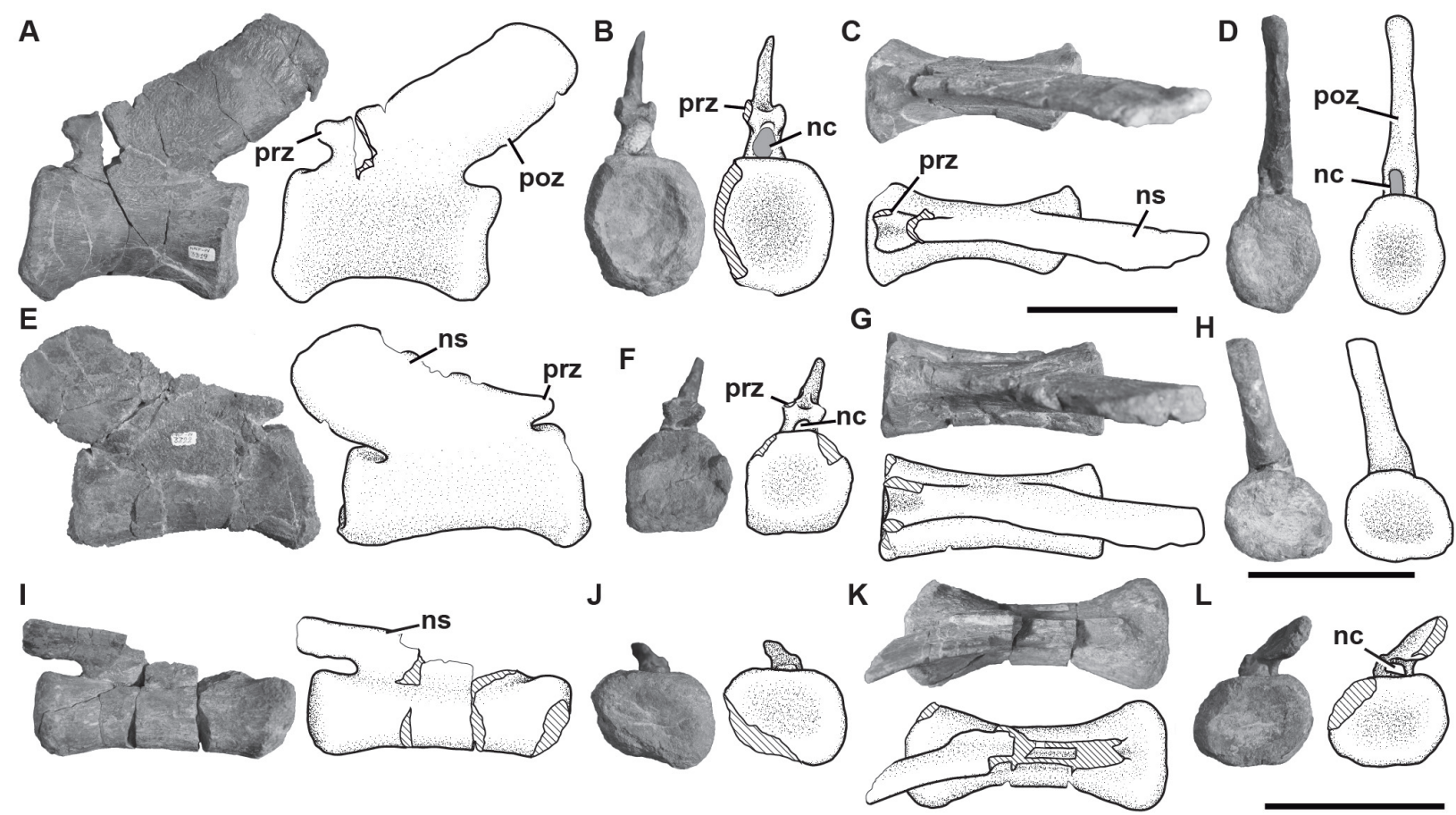

FIGURE 25. Posterior caudal vertebrae of Bagualia alba. A-D, caudal vertebra MPEF-PV 3319 in left lateral (A), anterior (B), dorsal (C), and posterior (D) views. E-H, caudal vertebra MPEF-PV 3322 in right lateral (E), anterior (F), dorsal (G), and posterior (H) views. I-L, caudal vertebra MPEF-PV 3331 in right lateral (I), anterior (J), dorsal (K), and posterior (L) views. Abbreviations: nc, neural canal; ns, neural spine; poz, postzygapophysis; prz, prezygapophysis. Hatched pattern represents broken surfaces and grey form represents sediment. Scale bar equals $10 \mathrm{~cm}$.

being slightly larger. The anterior haemal arches have a straight ventral blade that is twice as long anteroposteriorly as lateromedially. On the posterior surface of the ventral blade of MPEF-PV 3356, there is a ridge extending along the midline of the shaft (Figure 26A), while in MPEF-PV 3357 and MPEF-PV 3351, the ridge is also seen on the anterior surface (Figure 26C-D). Striated ridges along the midline of the haemal shafts are also present in other sauropods (e.g., Tazoudasaurus, Cetiosaurus, Patagosaurus, Spinophorosaurus; Allain and Aquesbi, 2008, figure 19E; Upchurch and Martin, 2002; Bonaparte, 1986, figure 48; GCP-CV 4229). The distal end of the ventral blade is expanded anteroposteriorly and compressed mediolaterally in all arches except the posteriormost one preserved, which has a thin distal blade (Figure 26L). Towards the posterior haemal arches, the ventral blade is shortened and curved posteriorly (e.g., Figure 26G, $\mathrm{I}, \mathrm{K})$. Also, the posterior haemal arches have a thickening of their arms at the beginning of the distal plate. This thickening has a dorsoventrally striated lateral surface (e.g., Figure $26 \mathrm{H}-\mathrm{J}, \mathrm{L}-\mathrm{M}$ ).

\section{DISCUSSION}

The axial skeleton of sauropods is very informative, due to the high morphological variability in their vertebrae (Bonaparte, 1999). Vertebral features contribute to around a third of the total characters used in most phylogenetic analyses of sauropods that have been published (e.g., Wilson, 2002; Upchurch et al., 2004; Mannion et al., 2019a; Pol et al., 2020). These features not only encompass the large number of differences between eusauropods and basal sauropodomorphs, but also the numerous changes that occurred in this part of the skeleton in the main lineages of Eusauropoda. The axial remains of Bagualia allow us to study the morphological differences between early eusauropod taxa and their outgroups, and to trace character evolution among early sauropods. We carried out a phylogenetic analysis (see Materials and Methods, Phylogenetic Analysis, and the Appendix 1 and 2 for details) to outline the most relevant aspects regarding the early evolution of the axial skeleton in sauropodomorphs, in order to understand the origins of one of their most striking anatomical features: the long neck. 


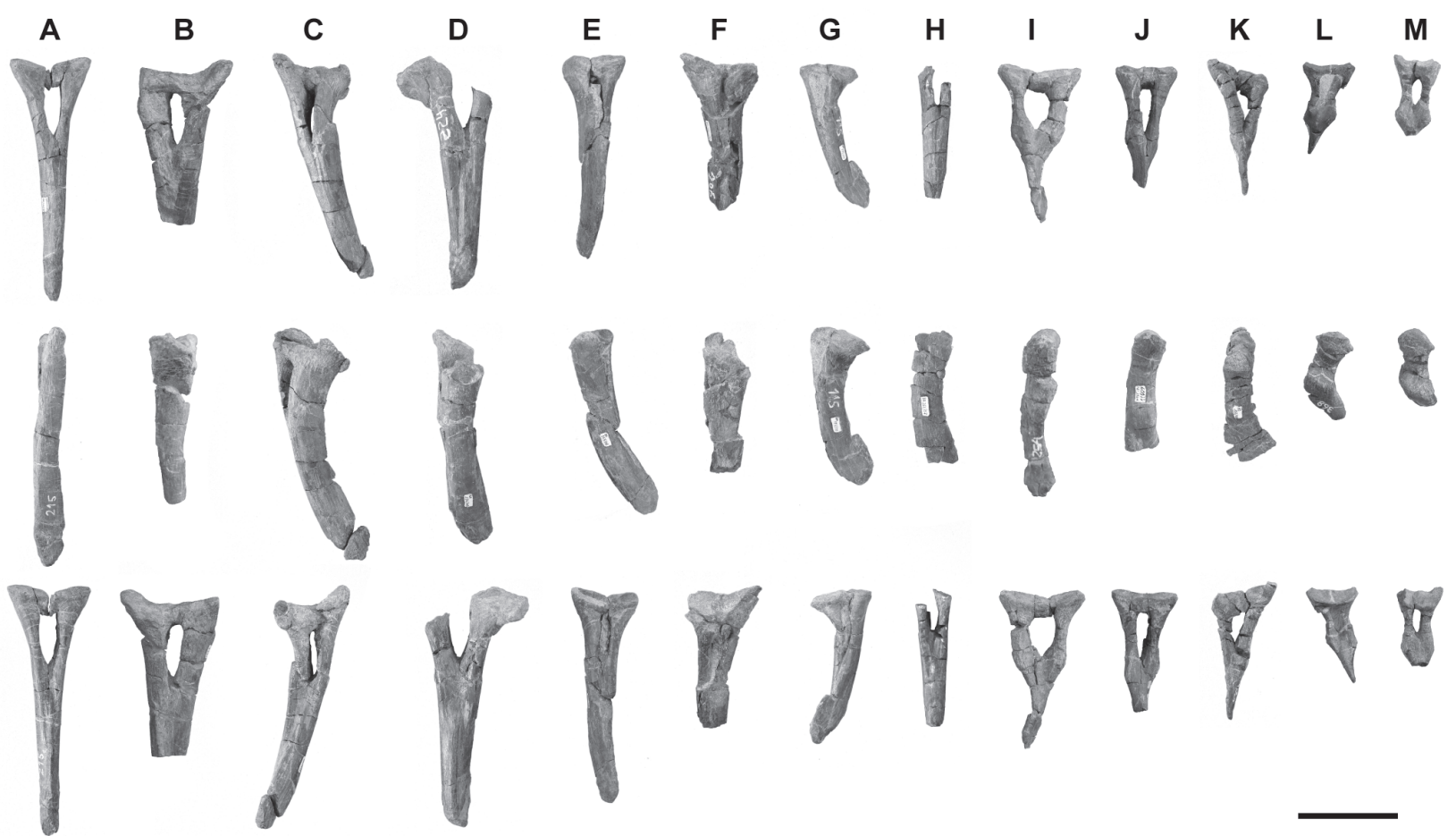

FIGURE 26. Haemal arches of Bagualia alba (from top to bottom) in anterior, left lateral and posterior views. A, MPEF-PV 3356; B, MPEF-PV 11025; C, MPEF-PV 3351; D, MPEF-PV 3357; E, MPEF-PV 3353; F, MPEF-PV 3355; G, MPEF-PV 3352; H, MPEF-PV 3359; I, MPEF-PV 3358; J, MPEF-PV 11009; K, MPEF-PV 3354; L, MPEF-PV 3390; M, MPEF-PV 11010. From left to right the haemal arches are ordered as proximal to distal elements. Scale bar equals $10 \mathrm{~cm}$.

\section{Phylogenetic Analysis}

In agreement with the results of Pol et al. (2020), Bagualia is recovered within a monophyletic group in the base of Eusauropoda (Figure 27; Figures 1-2 in Appendix 1). Taking polarity relative to Neosauropoda, this group is more derived than Barapasaurus, but more basal than Cetiosaurus and Mamenchisauridae. The positions of the main subclades within Sauropodomorpha are similar to that obtained by most recent phylogenetic analyses focused on basal sauropodomorphs (e.g., Pol and Powell, 2007; Otero et al., 2015; Zhang et al., 2018) and eusauropods (e.g., Xing et al., 2015; Mannion et al., 2019a; Royo Torres et al., 2021). Compared to recent studies with broad taxonomic sampling of sauropod taxa (e.g., Mannion et al., 2019a; Royo Torres et al., 2021), our analysis has greater representation (31 operational taxonomic units) for non-eusauropod sauropodomorphs, allowing more robust testing of evolutionary patterns at the base of and outside Eusauropoda (Appendix 2).

The monophyletic group containing Bagualia also contains other taxa recorded during the late
Early and Middle Jurassic (Spinophorosaurus, Nebulasaurus, and Patagosaurus; Figure 27; Figure 2 in Appendix 1). The monophyly of this group is supported by the following synapomorphies: up to three replacement teeth per alveolus in the dentary (present in Bagualia and Patagosaurus); cervical vertebrae with epipophyses that project posteriorly as thin prongs (convergent in Pulanesaura, Turiasauria, and some neosauropods); cervical vertebrae with pre-epipophyses (convergent in Mamenchisaurus and some turiasaurians and neosauropods); anteriormost dorsal vertebrae with epipophyses (present in Bagualia and Patagosaurus and convergent in Mamenchisaurus, Moabosaurus, Jobaria, and some neosauropods); posterior dorsal vertebrae with $\mathrm{mSPOL}$ (convergent in several neosauropods); first posterior caudal vertebrae with the neural spine strongly directed posteriorly (also present in Tazoudasaurus, Mamenchisaurus, and some neosauropods); and postacetabular process of the ilium between 40$100 \%$ of the distance between the pubic and ischial peduncles (widespread in Neosauropoda). Within this group, Patagosaurus is recovered as the sister taxon to a polytomy composed by Bagualia, Nebu- 


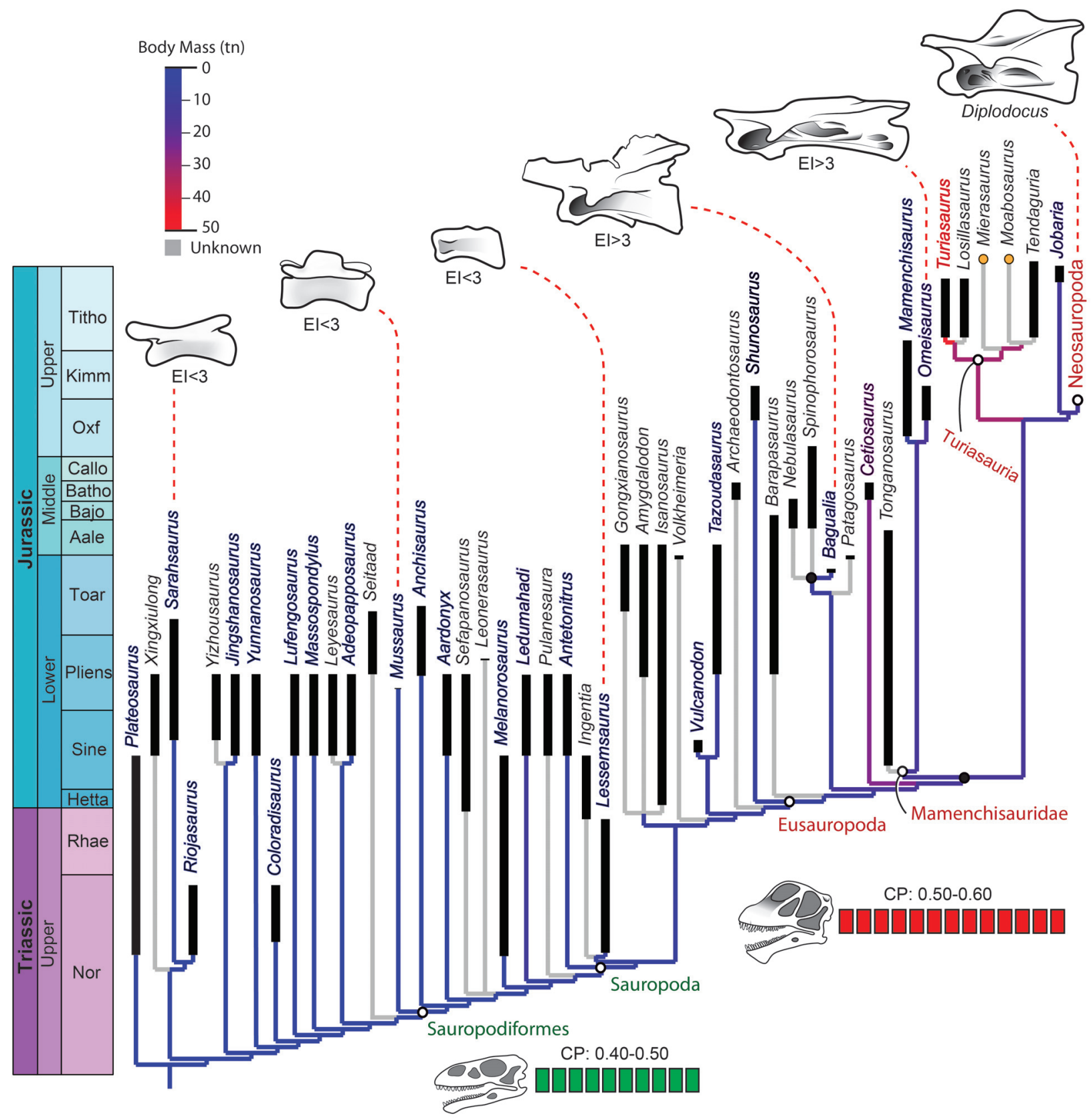

FIGURE 27. Simplified reduced consensus tree showing the calibrated phylogeny of non-neosauropod sauropodomorphs with the optimization of axial characters of the neck (i.e., CP ratio, El index, Body Mass, and pleurocoels). Black circle indicates the change in the El. Orange circles indicate Cretaceous taxa.

lasaurus, and Spinophorosaurus, which is supported by the following synapomorphies: D-shaped tooth crown mesiodistally narrow along its apical half (widespread amongst sauropods, also an ambiguous synapomorphy of Turiasauria); middle and posterior dorsal vertebrae with the height of the neural arch (below the postzygapophyses) less than the height of the centrum (also present in Moabosaurus, Turiasaurus, Jobaria, and several neosauropods); femoral head directed dorsally above the level of the greater trochanter (also present in Shunosaurus, Moabosaurus, and some neosauropods); femur with distal condyles subequal in transverse breadth (widespread character in non eusauropod sauropodomorphs); and femur with the fourth trochanter near the midline of the posterior surface of the shaft (also present in Shunosaurus, Mamenchisauridae, and some neosauropods). 
Within non-neosauropod Eusauropoda, we also obtained two other subclades previously recognized by other authors, Mamenchisauridae and Turiasauria (Figure 27). Mamenchisauridae is found in a more derived position than the lineage formed by Bagualia and related taxa. This position is similar to that obtained historically for this group, contrasting with a more basal position recently obtained by Mannion et al. (2019a) and Royo Torres et al. (2021). This difference could be due to the fact that our taxon sampling outside Eusauropoda is greater, which could modify the optimizations in the most basal nodes within Eusauropoda. In our analysis, Mamenchisauridae is composed of derived eusauropods from the Jurassic of China, with Tonganosaurus recovered as the sister taxon of a clade formed by Omeisaurus and Mamenchisaurus. A close relationship between Omeisaurus and Mamenchisaurus has been traditionally recognized by other authors (e.g., Allain and Aquesbi, 2008; Remes et al., 2009; Carballido and Sander, 2014; Tschopp et al., 2015; Carballido et al., 2020), although some recent analyses have recovered Omeisaurus outside of Mamenchisauridae (e.g., Mannion et al., 2019a; Moore et al., 2020; Royo Torres et al., 2021). Another diverse clade of basal eusauropods is Turiasauria, which was recovered as a lineage more closely related to Neosauropoda than Mamenchisauridae (Figure 27). This clade, defined by Royo Torres et al. (2006), is composed of Jurassic and Cretaceous taxa from Europe (e.g., Losillasaurus, Turiasaurus), North America (e.g., Moabosaurus, Mierasaurus), and Africa (i.e., Tendaguria). Within Turiasauria, Turiasaurus and Losillasaurus form the sister clade to a more diverse subclade including Mierasaurus, Moabosaurus, and Tendaguria. The strict consensus renders this latter subclade as a polytomy when Zby and Haestasaurus are included (Figure 1 in the Appendix 1). The reduced consensus shows possible positions of Zby within Turiasauria, recovered mostly within the clade composed of Mierasaurus, Moabosaurus, and Tendaguria (Figure 2 in Appendix 1). Another possible position for Zby is within the subclade composed of Turiasaurus and Losillasaurus, as has been suggested by other analyses (e.g., Mannion et al., 2019a; Royo Torres et al., 2021). In the case of Haestasaurus, this taxon can take four possible positions, one within Turiasauria, related to Moabosaurus and Tendaguria, and the other three within Neosauropoda, as a dicraeosaurid or as a basal macronarian. The position of Haestasaurus within Turiasauria is also seen in the equal weights analysis of Mannion et al. (2019a), although that analysis recovers it together with $\mathrm{Bel}-$ lusaurus and Tehuelchesaurus (here, these taxa are recovered as basal macronarians). In the analysis under implied weighing of Mannion et al. (2019a) and Royo Torres et al. (2021), Haestasaurus is recovered outside of Turiasauria and Bellusaurus and Camarasaurus were recovered as basal eusauropods instead of basal macronarians, as recovered here (Figure 2 in the Appendix 1 ) and by other authors (e.g., Canudo et al., 2008; Carballido et al., 2020). The alternative position of Haestasaurus as a basal macronarian is also similar to that obtained by other authors (e.g., Upchurch et al., 2015; Moore et al., 2020). Our analysis recovers Jobaria as the sister taxon of Neosauropoda, a position shared with various other authors (e.g, Remes et al., 2009; Carballido and Sander, 2014; Carballido et al., 2020; Holwerda et al., 2021), but a more derived placement than that recently obtained by Mannion et al. (2019a) and Royo Torres et al. (2021), where it is recovered as a basal eusauropod between Mamenchisauridae and Turiasauria.

The main clades within Neosauropoda have been recovered as traditionally recovered by other authors (e.g., Salgado et al., 1997; Wilson, 2002; Upchurch et al., 2004), but as it is not our objective to resolve their relationships here, we will not focus on topological variations within this diverse group. Based on the results of the phylogenetic analysis, certain axial characters related to neck lengthening have been optimized (Table 7, Figures 3-11 in Appendix 1) and are discussed below to understand cervical elongation in Sauropodomorpha.

\section{Neck Lengthening}

Neck elongation is a hallmark change in the evolution of sauropods, with major ecological implications (Sander et al., 2011; Barrett, 2014). Three possible changes to the ancestral neck morphology have been implicated in the lengthening of the sauropodomorph cervical series: 1) appearance of new elements in the cervical region; 2) conversion of a dorsal vertebra and its incorporation into the cervical region; and 3) elongation of pre-existing cervical vertebrae (Upchurch, 1994; Wilson and Sereno, 1998). Changes 1 and 3 are the result of somitogenetic and somatic growth processes (meristic change), whereas point 2 (homeotic change; Kessel and Gruss, 1991) is possibly due to changes in the expression of hox genes during the early stages of embryonic development (Burke et al., 1995; Richardson et al., 1998; Carroll, 2005). The expression pattern of hox genes is correlated 
with changes in the vertebral morphology, which in turn have been used to group vertebrae in different axial regions and subregions (Böhmer et al., 2015). To explore the processes that could have produced longer necks in sauropods, we analyze here two types of mechanisms: addition of new cervical elements to the neck (both due to somitogenesis or regionalization) and elongation of the cervical elements of the neck.

Addition of new cervical elements to the neck. Most studies concerning axial development in archosaurs are based on observations in the embryonic stages in crocodiles and birds (Mansfield and Abzhanov, 2010; Böhmer et al., 2019; Bui and Larsson, 2021). Since we do not have preserved complete embryonic stages of sauropodomorphs, we use the vertebral count of fossil specimens with complete (or almost complete) axial series to infer possible mechanisms of neck elongation in the phylogeny, as conducted previously for other groups (Müller et al., 2010). The presacral formula of basal sauropodomorphs is, in general, 10 cervicals and 14 dorsal vertebrae, whereas the general pattern in eusauropods is that they usually have more cervical and fewer dorsal vertebrae. For example, the early eusauropod Shunosaurus has 13 cervicals and 13 dorsals (1313 presacral formula) and Spinophorosaurus has a 13-12 presacral formula (Remes et al., 2009). Other basal eusauropods, such as Omeisaurus had even more cervicals, reaching a 16-12 or 1712 presacral formula (Wilson and Sereno, 1998). This increase was paralleled in Neosauropoda, in which there are lineages that evolved long necks, such as Diplodocidae with 15 cervicals and 10 dorsals, and some titanosaurs such as Futalognkosaurus that probably had 14 cervicals and 10 dorsals (Wilson, 2002; Calvo et al., 2007). Meristic and homeotic changes are not correlated, so the ratio between the cervical number and the total presacral vertebral number (CP ratio) of the taxa can give information about whether variation in the axial formula is due to differences in regionalization mediated by hox genes or by somitogenesis (Müller et al., 2010).

The first radiation of Sauropodomorpha in the Norian-Rhaetian is characterized by low CP values (0.39-0.40; Figure 27; Figure 3 in the Appendix 1), as evidenced by taxa with a complete axial skeleton such as Plateosaurus or Riojasaurus. From this radiation, many lineages of sauropodomorphs continued to emerge in the earliest Jurassic, diversifying further into different clades. The CP values are maintained as relatively low
(0.40-0.42) during this subsequent diversification, as exemplified by many sauropodomorphs recorded during this time (e.g., Massospondylus, Lufengosaurus, Sarahsaurus, Yunnanosaurus, Yizhousaurus; Table 7 in the Appendix 1), which have a presacral formula of 10-14 and retained the ancestral condition of a total of 24-25 presacral vertebrae present in early plateosaurians. A notable exception is Adeopapposaurus, which has a higher $\mathrm{CP}$ ratio $(0.46)$ due to the presence of 11 cervicals but the same presacral number as closely related massospondylid taxa. This would represent a first pulse in the addition of a new element to the neck, likely by changes in the expression of homeotic genes.

An important group that radiated during the Early Jurassic is Sauropodiformes, which is represented by Melanorosaurus with a CP ratio similar to that of early plateosaurians (0.38). Its condition is optimized as a reversal to the ancestral sauropodomorph condition in this terminal branch, but the lack of information on other early sauropodiforms precludes determining whether this is a more generalized pattern for the group. In fact, the lack of information on CP values extends from early sauropodiforms to the base of Eusauropoda, in which the range of CP values is larger (above 0.5). This gap in the knowledge of CP ratio includes lessemsaurids (basal sauropods sensu Yates, 2007) and other sauropodiform or early sauropod taxa (e.g., Gongxianosaurus, Pulanesaura, Vulcanodon, Tazoudasaurus) that are critical for understanding the origin of the increase in $\mathrm{CP}$ ratio in Eusauropoda. More complete axial skeletons of any of these taxa would provide important information on changes happening during the latest Triassic or the Early Jurassic prior to the Toarcian.

The radiation of Eusauropoda (during the Toarcian and earliest Middle Jurassic; see Pol et al., 2020) is characterized by CP ratios greater than 0.5 (Figure 27). Most early eusauropods have a presacral formula of 13-12 (e.g., Cetiosaurus, Patagosaurus, Spinophorosaurus). These CP ratios are high with respect to the ancestral condition, but the presacral number is similar (i.e., 25), therefore, we can infer that the appearance of a new cervical element in the earliest eusauropods was a result of changes in the expression of homeotic genes and a change in the regionalization of some elements. A lineage of non-neosauropod eusauropods recorded in China during the Late Jurassic (e.g., Mamenchisaurus and Omeisaurus, here recovered forming the clade Mamenchisauridae) has even higher $\mathrm{CP}$ ratios (close to 
$0.6)$ and a larger number of presacral vertebrae with respect to the ancestral condition, so in this clade there were likely also meristic changes.

Within Neosauropoda, a CP ratio of 0.5 is present in most clades (Dicraeosauridae, Macronaria, Camarasauromorpha, Brachiosauridae). However, the case of Diplodocidae is different, because it has higher $\mathrm{CP}$ ratios, similar to mamenchisaurid eusauropods (greater than 0.6), but unlike these, the presacral number is equal to that the ancestral condition, so probably only homeotic changes operated in the generation of very long necks in this clade (e.g., Diplodocus, Apatosaurus, Barosaurus). Other taxa within certain clades of Neosauropoda have indications of both types of change, as in the cases of Euhelopus or the titanosaurian Rapetosaurus, indicating that evolutionary changes in the necks of neosauropods probably followed a complex and dynamic history with extensive convergences and parallelisms.

In the evolution of Sauropodomorpha, the first cases of appearance of new cervical elements seem to be the result of presacral regionalization, likely caused by changes in the expression of homeotic genes. During the first 40 million years of sauropodomorph evolution, there seems to be a single case of this type of evolutionary change (Adeopapposaurus), suggesting the presence of a developmental constraint that maintained cervical count. The evolutionary radiation of Eusauropoda (or perhaps Sauropoda, although more data at the base of this clade are needed) seems to be characterized by a release of this constraint, and consequently more diverse configurations of the cervical region. This evolutionary plasticity probably reflects the presence of both homeotic and meristic changes operating in the presacral region, processes that also characterize the evolutionary dynamic within neosauropods.

Elongation of cervical centra. The increase in the number of cervical vertebrae in Sauropodomorpha was coupled with an elongation of the individual cervical vertebrae to produce the long neck of sauropods (Wilson and Sereno, 1998). The postaxial vertebrae of Bagualia increase in length towards the middle of the neck, which is evident not only from the total length of each centrum, but also by their El (see Table 3 in Appendix 1). This is the usual pattern in sauropodomorphs, where the El peaks at the mid-cervicals and decreases anterior and posterior to this point (Carballido and Sander, 2014). Since their radiation during the Late Triassic-Early Jurassic, basal sauropodomorphs had mid-cervical centra about two to three times longer than tall, except for a few taxa in which the El was much higher (e.g., Leyesaurus: 5.12; Adeopapposaurus: 4.11; see Figure 4 in Appendix 1). This shows that at the beginning of the sauropodomorph radiation (likely in the Norian), there was an initial event of elongation of the cervical centra in taxa that have their neck composed of fewer elements than the trunk (low CP).

Despite the absence of complete axial series in early sauropodiforms, the El measured in the fifth cervical vertebra is similar than those of more basal sauropodomorphs (e.g., Mussaurus: 2.31; Leonerasaurus: 2.75; Ingentia: 3.29; Lessemsaurus: 2; Table 7 in Appendix 1). The early sauropods from the Early Jurassic have El values that remain within the range of basal sauropodomorphs (e.g., Amygdalodon: 3.3; Tazoudasaurus: 2.5). Similarly, almost all early eusauropods from the late Early to Middle Jurassic have El values that are similar to those present in basal sauropodomorphs (e.g., Shunosaurus: 2.64; Patagosaurus: 2.1; Spinophorosaurus: 3 ). The sole exception is Bagualia, which has a greater elongation $(\mathrm{aEI}=4.6)$, here interpreted as convergently acquired with the long neck of later-diverging eusauropods, such as the mamenchisaurids from the Late Jurassic of China (e.g., Omeisaurus: 5.96; Mamenchisaurus: 3.76).

The basal nodes of Neosauropoda maintain these relatively low El values in their mid-cervical vertebrae (e.g., Dicraeosaurus: 2.58; Amargasaurus: 2.20; Camarasaurus: 3.50). However, within this clade there is a clear increase in cervical elongation inferred at the base of Diplodocoidea (e.g., Diplodocus: 4.95; Suuwassea: 4.73; Nigersaurus: 4) and Titanosauriformes (e.g., Giraffatitan: 4.6; Malawisaurus: 6.48; Rapetosaurus: 6.43), or possibly more basal nodes within Macronaria (e.g., Haplocanthosaurus: 4.22). Thus, the case of Bagualia represents an early, independent elongation event during the radiation of eusauropods in the late Early Jurassic, preceding three similar events occurring in Late Jurassic sauropods (e.g., mamenchisaurids, diplodocoids, titanosauriformes), some of which coupled this elongation with an increase in the number of cervical vertebrae.

\section{Pneumaticity}

Pneumaticity in eusauropod presacral vertebrae takes different forms and can be developed both internally (e.g., Wedel, 2003, 2007) and externally (e.g., lateral fossae, pleurocoels; Wilson et al., 2011; Carballido and Sander, 2014), affecting the centrum and the neural arch of presacral verte- 
brae, which usually have a complex pattern of bony laminae. Furthermore, pneumaticity can even be present in the sacrum and the anterior and middle caudal vertebrae (Schwarz et al., 2007; Wedel et al., 2000; Wedel 2003, 2007, 2009; Cerda et al., 2012). The presacral vertebrae of non-eusauropod sauropodomorphs, in general, lack large internal pneumatic structures (Wedel, 2005; Figure 6 in Appendix 1). This is the case in Bagualia and the earliest eusauropods, which have cervical, dorsal, and caudal vertebrae that lack internal cavities.

Cervical vertebrae. In general, basal sauropodomorphs lack pleurocoels in cervical vertebrae (Wilson and Sereno, 1998; Wedel, 2007). A marked pleurocoel appears at least in the noneusauropod sauropod Volkheimeria from the end of the Early Jurassic, although the oldest record is present in Bagualia, found in a somewhat lower stratigraphic level in the same formation. Within Eusauropoda, the pleurocoels are already evident in most of the Middle and Late Jurassic taxa (e.g., Spinophorosaurus, Shunosaurus, Turiasaurus; Figure 8 in the Appendix 1). In general, pleurocoels in early eusauropods recorded during the Early to Middle Jurassic are simple, without any division (Figure 9 in the Appendix 1). Only in the eusauropod Patagosaurus is there a posteroventrally oriented crest that originates just below the diapophysis and divides the pleurocoel into two subfossae (Holwerda et al., 2021), a feature that is incipiently present in Spinophorosaurus. More derived eusauropods (e.g., Jobaria) and several neosauropods have the same degree of development as in Patagosaurus. Furthermore, some eusauropods from the Late Jurassic show pleurocoels with several septa that divide them into numerous subfossae (e.g., Mamenchisaurus, Moabosaurus), a derived condition convergently acquired in some neosauropod lineages, such as Diplodocidae (e.g., Apatosaurus, Barosaurus) and some brachiosaurids (e.g., Giraffatitan, Brachiosaurus). This distribution indicates that although the generalized and plesiomorphic condition for early eusauropods in the Early-Middle Jurassic is the presence of simple pleurocoels, there were independent events of increased complexity in the pneumatization of the cervical centra since the Early Jurassic, as evidenced by Patagosaurus. Subsequently, multiple lineages further develop more complex pneumatization of the cervical centra during the Late Jurassic, as observed in several neosauropod lineages and mamenchisaurids. Regarding the internal pneumaticity of the cervical centra, this begins to be evident from the Late
Jurassic with Mamenchisaurus, and expands widely during the Cretaceous with the turiasaurian Moabosaurus and most of neosauropods, where a great diversity of internal spaces is present (e.g., camellate, camerate, polycamerate).

The cervical neural arches of Bagualia have pneumatic structures, but the origin of these features has a deeper origin in the evolution of Sauropodomorpha. Several non-eusauropod sauropods recorded as early as the Late Triassic have incipient pneumatic structures in the neural arch, such as fossae with internal subfossae in the CDF and the PRCDF of posterior cervical vertebrae (e.g., Thecodontosaurus, Ingentia, Lessemsaurus; Yates, 2003; Apaldetti et al., 2018) or anteriormost dorsal vertebrae (e.g., Lessemsaurus; Pol and Powell, 2007). The cervical neural arches of eusauropods, however, have a greater development of laminae and fossae, as seen in several late Early Jurassic taxa (e.g., Barapasaurus, Patagosaurus). Bagualia has the greatest development of neural arch laminae and fossae among Early Jurassic eusauropods. This is evidenced by the presence of accessory laminae within the SDF in middle and posterior cervical vertebrae, which are also recorded later in the Late Jurassic and Early Cretaceous turiasaurs (e.g., Mierasaurus, Moabosaurus) and some neosauropod clades (e.g., Rebbachisauridae, Macronaria). Other eusauropod lineages further develop neural arch pneumaticity, as shown by the presence of pits on the lateral surface of the prezygapophyseal process (e.g., Omeisaurus, Moabosaurus, Apatosaurus).

Dorsal vertebrae. Pleurocoels vary in their extent along the dorsal series in Early Jurassic sauropods. Whereas pleurocoels are restricted to the anterior dorsal vertebrae in some taxa (e.g., Tazoudasaurus, Bagualia), some eusauropods during the late Early Jurassic have pleurocoels throughout the entire dorsal series (Patagosaurus; Holwerda et al., 2021). Subsequently, this condition is widely present in most eusauropods during the MiddleLate Jurassic and Cretaceous (e.g., Cetiosaurus, Mamenchisaurus, Turiasaurus; Figure 9 in Appendix 1). As in the cervical vertebrae, the dorsal centra develop internal spaces only in the derived eusauropods recorded during the Late Jurassic and Cretaceous. On the other hand, internal pneumaticity of the dorsal neural arches first appear in some eusauropods during the Early and Middle Jurassic as neural cavities (e.g., Barapasaurus, Patagosaurus, Cetiosaurus; Upchurch and Martin, 2003; Wedel, 2005; Holwerda et al., 2021). 
The cervical region of early sauropodomorphs remained relatively static during the first radiation of the group towards the end of the Triassic. In general, non-eusauropod sauropodomorphs have simple cervical vertebrae that lack both internal and external pneumatic structures, with a rudimentary laminar development and a relatively short neck both in terms of the vertebral count (10 cervical vertebrae; CP: $0.40-0.50$ ) and the elongation of each cervical vertebra $(E I<3)$. These characteristics are maintained throughout the diversification of Sauropodiformes between the Late Triassic and the Early Jurassic, despite a marked increase in body size marking the appearance of taxa with weights greater than 5 tons (e.g., Lessemsaurus, Ledumahadi, Antetonitrus).

The most notable changes in the neck of Sauropodomorpha occurred during the radiation of Eusauropoda (Figure 27). The eusauropods recorded by the end of the Early Jurassic and Middle Jurassic show a great increase in body size, with several species that exceeded 10 tons. This increase was accompanied by changes in the neck, such as the development of external structures related to vertebral pneumaticity (i.e., the appearance of pleurocoels in the centrum and a greater development of the laminae in the neural arch; Wedel, 2007; Rauhut et al., 2011). These changes are accompanied by an overall elongation of the neck (Wedel, 2006) both in terms of the number of elements (13-16 cervical vertebrae; CP: $0.50-0.60)$ and by the greater elongation of each individual vertebra $(3<\mathrm{EI}<5)$.

More derived eusauropods recorded from the Late Jurassic, such as the Turiasauria or Neosauropoda, further develop the pleurocoel, which becomes deeper with discrete edges on all sides (Figure 27). These features are maintained throughout Neosauropoda, although a reduction of these occurs later in Eutitanosauria. Another important, late appearing feature is the appearance of laminae within the pleurocoels, which are observed in mamenchisaurids (e.g., Omeisaurus, Mamenchisaurus), Jobaria, and Neosauropoda (a reversal of this feature is also present in eutitanosaurians with undivided pleurocoels). Finally, the internal pneumaticity of the cervical vertebrae is exclusive to Neosauropoda, where large internal cavities are observed in diplodocoids and macronarians, and a camellate-type system is present in diplodocids and titanosauriforms (Britt, 1997; Wedel, 2003).

Despite having a fairly homogeneous body plan and body mass exceeding 10 tons, eusauro- pods show high variability in the anatomical details of the neck, which do not always correlate with variations in their body size. In summary, current evidence indicates that: early sauropodomorphs maintained a plesiomorphic neck configuration, and cases of neck elongation among basal sauropodomorphs occurred in the absence of pneumatic structures and at small body size (e.g., Adeopapposaurus); sauropodomorphs with longer necks appear in lineages with large body size (greater than 10 tons) and are frequently associated with pneumatic structures (e.g., mamenchisaurids); and having a large body size, however, does not require the presence of a long neck or of sophisticated pneumatic structures, as exemplified by taxa with large body size (6-14 tons) and relatively short necks with reduced pneumaticity (e.g., lessemsaurids, dicraeosaurids).

The Toarcian age of Bagualia reveals the timing of appearance of some of these axial features, such as the presence of pleurocoels and accessory laminae in the neural arch of the cervical vertebrae, as well as great elongation of their centra (Figure 27). This morphology indicates an early lengthening event of a pneumatic neck in animals with large body size (10 tons) immediately after the Pliensbachian-Toarcian crisis recorded in Patagonia. A long neck allowed covering a larger feeding area, reaching vegetation that is not easily accessible to other herbivores (Dodson, 1990; Christian and Szemski, 2007). The increase in the pneumaticity of the vertebrae has been noted to reduce weight and facilitate heat dissipation (Sander et al., 2011). After the volcanic event recorded in Patagonia during the Pliensbachian-Toarcian (180-185 $\mathrm{Ma}$ ), the flora changed markedly, being dominated by Araucariaceae, Cupressaceae, and Cheirolepidiaceae conifers (Cúneo et al., 2013; Figari et al., 2015). These plants are abundant in the global Jurassic record and likely were important components of the diet of high browser sauropods like Bagualia due to their high energy content (Hummel et al., 2008). Teeth with a spoon shape and thick enamel, as described in Bagualia (Pol et al., 2020), allowed the intake of this type of hard vegetation (Upchurch and Barret, 2000; Sellers et al., 2019). Bagualia represents the first record of the axial characters that enabled the Eusauropoda to be a successful group in the face of the new environmental conditions that arose after the Pliensbachian-Toarcian crisis in Patagonia, which possibly helped them to become the dominant group of herbivores in Middle Jurassic ecosystems worldwide. 


\section{ACKNOWLEDGEMENTS}

We thank the personnel of the MEF for the preparation of the materials studied in the present work. Comments made by anonymous reviewers and the editor helped improve an earlier version of this paper. The authors gratefully acknowledge the financial support provided by PICT 2014-1288 y PICT 2020-03834 (to DP) and PICT 1925 (to JLC).

\section{REFERENCES}

Allain, R. and Aquesbi, N. 2008. Anatomy and phylogenetic relationships of Tazoudasaurus naimi (Dinosauria, Sauropoda) from the late Early Jurassic of Morocco. Geodiversitas, 30:345-424.

Allain, R., Aquesbi, N., Dejax, J., Meyer, C.A., Monbaron, M., Montenat, C., Richir, P., Rochdy, M., Russell, D.A., and Taquet, P. 2004. A basal sauropod dinosaur from the Early Jurassic of Morocco. Systematic Paleontology (Vertebrate Paleontology), 3:199-208. https://doi.org/10.1016/j.crpv.2004.03.001

Apaldetti, C., Martinez, R.N., Alcober, O.A., and Pol, D. 2011.A new basal sauropodomorph (Dinosauria: Saurischia) from Quebrada del Barro Formation (Marayes-El Carrizal Basin), northwestern Argentina. PLoS ONE, 6:e26964. https://doi.org/10.1371/journal.pone.0026964

Apaldetti, C., Martínez, R.N., Cerda, I.A., Pol, D., and Alcober, O. 2018. An early trend towards gigantism in Triassic sauropodomorph dinosaurs. Nature Ecology and Evolution, 2(8):1227. https://doi.org/10.1038/s41559-018-0599-y

Bandyopadhyay, S., Gillette, D.C., Ray, S., and Sengupta, D.P. 2010. Osteology of Barapasaurustagorei (Dinosauria: Sauropoda) from the Early Jurassic of India. Palaeontology, 53:533-569. https://doi.org/10.1111/j.1475-4983.2010.00933.x

Barco, J.L. 2009. Sistemática e implicaciones filogenéticas y paleobiogeográficas del saurópodo Galvesaurus herreroi (Formación Villar del Arzobispo, Galve, España). Unpublished PhD Thesis, Universidad de Zaragoza, España.

Barrett, P.M. 2014. Paleobiology of herbivorous dinosaurs. Annual Review of Earth and Planetary Sciences, 42:207-230. https://doi.org/10.1146/annurev-earth-042711-105515

Becerra, M.G., Gomez, K.L., and Pol, D. 2017. A sauropodomorph tooth increases the diversity of dental morphotypes in the Cañadón Asfalto Formation (Early - Middle Jurassic) of Patagonia. Comptes Rendus Palevol, 16:832-840. https://doi.org/10.1016/j.crpv.2017.08.005

Benton, M.J., Juul, L., Storrs, G.W., and Galton, P.M. 2010. Anatomy and systematics of the prosauropod dinosaur Thecodontosaurus antiquus from the upper Triassic of southwest England. Journal of Vertebrate Paleontology, 20(1):77-108.

Böhmer, C., Rauhut, O.W., and Wörheide, G. 2015. Correlation between Hox code and vertebral morphology in archosaurs. Proceedings of the Royal Society B: Biological Sciences, 282(1810):20150077. https://doi.org/10.1098/rspb.2015.0077

Böhmer, C., Plateau, O., Cornette, R., and Abourachid, A. 2019. Correlated evolution of neck length and leg length in birds. Royal Society open science, 6(5):181588. https://doi.org/10.1098/rsos.181588

Bonaparte, J.F. 1979. Dinosaurs: A Jurassic assemblage from Patagonia. Science, 205(4413):1377-1379. https://doi.org/10.1126/science.205.4413.1377

Bonaparte, J.F. 1986. Les Dinosaures (Carnosaures, Allosauridés, Sauropodes,

Cétiosauridés) du Jurassique Moyen de Cerro Cóndor (Chubut, Argentine). Annales de Paléontologie, 72:325-386.

Bonaparte, J.F. 1999. Evolución de las vértebras presacras en Sauropodomorpha. Ameghiniana, 36:115-187.

Bonaparte, J.F. and Pumares, J.A. 1995. Notas sobre el primer cráneo de Riojasaurus incertus (Dinosauria, Prosauropoda, Melanorosauridae) del Triásico Superior de La Rioja, Argentina. Ameghiniana, 32:341-349.

Britt, B.B. 1997. Postcranial pneumaticity. Postcranial pneumaticity, 590-593. In Currie, P. J., and Padian, K. (eds.), The Encyclopedia of Dinosaurs. Academic Press, San Diego, CA. 
Britt, B.B., Scheetz, R.D., Whiting, M.F., and Wilhite, D.R. 2017. Moabosaurus utahensis n. gen., n. sp., a new sauropod from the Early Cretaceous (Aptian) of North America. Contributions from the Museum of Paleontology, 32(11):189-243.

Buffetaut, E., Suteethorn, V., Cuny, G., Tong, H., Le Loeuff, J., Khansubha, S., and Jongautchariyakul, S. 2000. The earliest known sauropod dinosaur. Nature, 407:72-74.

Bui, H.N.N., and Larsson, H.C. 2021. Development and evolution of regionalization within the avian axial column. Zoological Journal of the Linnean Society, 191(1):302-321. https://doi.org/10.1093/zoolinnean/zlaa038

Burges, S.D., Bowring, S.A., Fleming, T.H., and Elliot, D.H. 2015. High-precision geochronology links the Ferrar large igneous province with early-Jurassic ocean anoxia and biotic crisis. Earth and Planetary Science Letters, 415:90-99. https://doi.org/10.1016/j.epsl.2015.01.037

Burke, A.C., Nelson, C.E., Morgan, B.A., and Tabin, C. 1995. Hox genes and the evolution of vertebrate axial morphology. Development, 121(2):333-346. https://doi.org/10.1242/dev.121.2.333

Cabrera, A. 1947. Un saurópodo nuevo del Jurásico de Patagonia. Notas del Museo de La Plata, 12:1-17.

Calvo, J.O., Porfiri, J.D., González Riga, B.J., Kellner, A.W.A. 2007. Anatomy of Futalognkosaurus dukei Calvo, Porfiri, González Riga and Kellner, 2007 (Dinosauria, Titanosauridae) from the Neuquén Group (Late Cretaceous), Patagonia, Argentina. Arquivos do Museu Nacional, Rio de JaElro, 65 (4):511-526.

Canudo, J.I., Royo-Torres, R., and Cuenca-Bescós, G. 2008. A new sauropod: Tastavinsaurus sanzi gen. et sp. nov. from the Early Cretaceous (Aptian) of Spain, Journal of Vertebrate Paleontology, 28(3):712-731. https://doi.org/10.1671/0272-4634(2008)28[712:ANSTSG]2.0.CO;2

Carballido, J.L. and Pol, D. 2010. The dentition of Amygdalodon patagonicus (Dinosauria: Sauropoda) and the dental evolution in basal sauropods. Comptes Rendus Palevol, 9:8393. https://doi.org/10.1016/j.crpv.2010.01.003

Carballido, J.L. and Sander, P.M. 2014. Postcranial axial skeleton of Europasaurus holgeri (Dinosauria, Sauropoda) from the Upper Jurassic of Germany: implications for sauropod ontogeny and phylogenetic relationships of basal Macronaria. Journal of Systematic Palaeontology, 12(3):335-387. https://doi.org/10.1080/14772019.2013.764935

Carballido, J.L., Scheil, M., Knötschke, N., and Sander, P.M. 2020. The appendicular skeleton of the dwarf macronarian sauropod Europasaurus holgeri from the Late Jurassic of Germany and a re-evaluation of its systematic affinities. Journal of Systematic Palaeontology, 18(9):739-781. https://doi.org/10.1080/14772019.2019.1683770

Carroll, S.B. 2005. Endless Forms Most Beautiful. Norton, New York.

Casanovas, M.L., Santafé, J.V., Sanz, J.L. 2001. "Losillasaurus giganteus", un nuevo saurópodo del tránsito Jurásico-Cretácico de la cuenca de "Los Serranos" (Valencia, España). Paleontologia i Evolució, (32):99-122.

Cerda, I.A., Salgado, L., and Powell, J.E. 2012. Extreme postcranial pneumaticity in sauropod dinosaurs from South America. PaläontologischeZeitschrift, 86(4):441-449. https://doi.org/10.1007/s12542-012-0140-6

Christian, A. and Dzemski, G. 2007. Reconstruction of the cervical skeleton posture of Brachiosaurus brancai Janensch, 1914 by an analysis of the intervertebral stress along the neck and a comparison with the results of different approaches. Fossil Record, 10(1):38-49. https://doi.org/10.5194/fr-10-38-2007

Chure, D., Britt, B.B., Whitlock, J.A., and Wilson, J.A. 2010. First complete sauropod dinosaur skull from the Cretaceous of the Americas and the evolution of sauropod dentition. Naturwissenschaften, 97(4):379-391. https://doi.org/10.1007/s00114-010-0650-6

Cooper, M.R. 1981. The prosauropod dinosaur Massospondylus carinatus Owen from Zimbabwe: its biology, mode of life and phylogenetic significance. Occasional papers of the National Museums and Monuments of Rhodesia. Series B. Natural sciences, 6(10):690-840.

Cooper, M.R. 1984. A reassessment of Vulcanodon karibaensis Raath (Dinosauria: Saurischia) and the origin of the Sauropoda. Palaeontologia Africana, 25:203-231.

Cúneo, R., Ramezani, J., Scasso, R., Pol, D., Escapa, I., Zavattieri, A.M., and Bowring, S.A. 2013. High-precision U-Pb geochronology and a new chronostratigraphy for the Cañadón Asfalto Basin, Chubut, central Patagonia: Implications for terrestrial faunal and floral evolution in Jurassic. Gondwana Research, 24(3-4):1267-1275.

https://doi.org/10.1016/j.gr.2013.01.010 
Curry Rogers, K.C. 2009. The postcranial osteology of Rapetosaurus krausei (Sauropoda: Titanosauria) from the Late Cretaceous of Madagascar. Journal of Vertebrate Paleontology, 29(4):1046-1086. https://doi.org/10.1671/039.029.0432

Dodson, P. 1990. Sauropod paleoecology, p. 402-407. In Weishampel, D.B., Dodson, P., and Osmolska, H. (eds.), The Dinosauria. University of California Press.

D'Emic, M.D. 2012. The early evolution of titanosauriform sauropod dinosaurs. Zoological Journal of the Linnean Society, 166(3):624-671.

Figari, C.E., Scasso, R.A., Cúneo, N.R., and Escapa, I.H. 2015. Estratigrafía y evolución geológica de la Cuenca de Cañadón Asfalto, Provincia del Chubut, Argentina. Latin American Journal of Sedimentology and Basin Analysis, 22:135-169.

Figari, E.G. 2005. Evolución tectónica de la cuenca de Cañadón Asfalto (Zona del valle medio del río Chubut). Doctoral dissertation, Universidad de Buenos Aires, Facultad de Ciencias Exactas y Naturales, Buenos Aires, Argentina.

Galton, P.M. 1976. Prosauropod dinosaurs (Reptilia: Saurischia) of North America. Postilla, 169:1-98.

Galton, P.M. 2000. The prosauropod dinosaur Plateosaurus Meyer, 1837 (Saurischia: Sauropodomorpha). I. The syntypes of $P$. engelhardti Meyer, 1837 (Upper Triassic, Germany), with notes on other European prosauropods with "distally straight" femora. Neues Jahrbuch für Geologie und Paläontologie - Abhandlungen, 216(2):233-275.

Gilmore, C.W. 1936. Osteology of Apatosaurus, with special reference to specimens in the Carnegie Museum. Memoirs of the Carnegie Museum,11:172-298.

Gomani, E.M. 2005. Sauropod dinosaurs from the early Cretaceous of Malawi, Africa. Palaeontologia Electronica, 8(1):1-37. https://palaeo-electronica.org/2005_1/gomani27/issue1_05.htm

Harris, J.D. 2006. The axial skeleton of the dinosaur Suuwassea emilieae (Sauropoda: Flagellicaudata) from the Upper Jurassic Morrison Formation of Montana, USA. Palaeontology, 49(5):1091-1121. https://doi.org/10.1111/j.1475-4983.2006.00577.x

Hatcher, J.B. 1901. Diplodocus (Marsh): Its osteolology, taxonomy, and probable habits, with a restoration of the skeleton. Memoirs of the Carnegie Museum, 1:347-355.

Hatcher, J.B. 1903. Osteology of Haplocanthosaurus, with description of a new species, and remarks on the probable habits of the Sauropoda and the age and origin of the Atlantosaurus beds. Memoirs of the Carnegie Museum, 2:1-72.

He, X-L., Li, K., and Cai, K.-J. 1988. The Middle Jurassic Dinosaur Fauna from Dashanpu, Zigong, Sichuan. Volume IV. Sauropod Dinosaurs (2). Omeisaurus tianfuensis. Sichuan Publishing House of Science and Technology, Chengdu, China, pp. 143. (In Chinese with English abstract)

Hesselbo, S.P., Gröcke, D.R., Jenkyns, H.C., Bjerrum, C.J., Farrimond, P., Bell, H.S. M., and Green, O.R. 2000. Massive dissociation of gas hydrate during a Jurassic oceanic anoxic event. Nature, 406(6794):392-395. https://doi.org/10.1038/35019044

Holwerda, F.M. and Pol, D. 2018. Phylogenetic analysis of Gondwanan basal eusauropods from the Early-Middle Jurassic of Patagonia, Argentina. Spanish Journal of Palaeontology, 33(2): 289-298.

Holwerda, F.M., Rauhut, O.W.M., and Pol, D. 2021. Osteological revision of the holotype of the Middle Jurassic sauropod dinosaur Patagosaurus fariasi Bonaparte, 1979 (Sauropoda: Cetiosauridae). Geodiversitas,43(16):575-643. https://doi.org/10.5252/geodiversitas2021v43a16

Hummel, J., Gee, C.T., Südekum, K.H., Sander, P.M., Nogge, G., and Clauss, M. 2008. In vitro digestibility of fern and gymnosperm foliage: implications for sauropod feeding ecology and diet selection. Proceedings of the Royal Society B: Biological Sciences, 275(1638):10151021. https://doi.org/10.1098/rspb.2007.1728

Irmis, R.B. 2007. Axial skeleton ontogeny in the Parasuchia (Archosauria: Pseudosuchia) and its implications for ontogenetic determination in archosaurs. Journal of Vertebrate Paleontology, 25:350-361. https://doi.org/10.1671/0272-4634(2007)27[350:asoitp]2.0.co;2

Janensch, W. 1929. Die Wirbelsäule der Gattung Dicraeosaurus. Palaeontographica, 2(7):35133.

Kessel, M. and Gruss, P. 1991. Homeotic transformations of murine vertebrae and concomitant alteration of Hox codes induced by retinoic acid. Cell, 67(1):89-104.

https://doi.org/10.1016/0092-8674(91)90574-I 
Ksepka, D.T. and Norell, M.A. 2006. Erketu ellisoni, a long-necked sauropod from Bor Guvé (Dornogov Aimag, Mongolia). American Museum Novitates, 3508:1-16. https://doi.org/10.1206/0003-0082(2006)3508[1:eealsf]2.0.co;2

Kutty, T.S., Chatterjee, S., Galton, P.M., and Upchurch, P. 2007. Basal sauropodomorphs (Dinosauria: Saurischia) from the Lower Jurassic of India: their anatomy and relationships. Journal of Paleontology, 81(6):1218-1240. https://doi.org/10.1666/04-074.1

Li, K., Yang, C.Y., Liu, J., and Wang, Z.X. 2010. A new sauropod from the Lower Jurassic of Huili, Sichuan, China. Vertebrata PalAsiatica, 3:185-202.

Lu, J., Li, T., Zhong, S., Azuma Y., Fujita, M., Dong, Z., and Ji, Q. 2007. New yunnanosaurid dinosaur (Dinosauria, Prosauropoda) from the Middle Jurassic Zhanghe Formation of Yuanmou, Yunnan Province of China. Memoir of the Fukui Prefectural Dinosaur Museum, 6:1-15.

Madsen, J., Mclntosh, J.S., Berman, D.S. 1995. Skull and atlas- axis complex of the Upper Jurassic sauropod Camarasaurus Cope (Reptilia: Saurischia). Bulletin of the Carnegie Museum of Natural History, 31:1-115.

Mannion, P.D. 2019. A turiasaurian sauropod dinosaur from the Early Cretaceous Wealden Supergroup of the United Kingdom. PeerJ, 7:e6348. https://doi.org/10.7717/peerj.6348

Mannion, P.D., Upchurch, P., Schwarz, D., and Wings, O. 2019a. Taxonomic affinities of the putative titanosaurs from the Late Jurassic Tendaguru Formation of Tanzania: phylogenetic and biogeographic implications for eusauropod dinosaur evolution. Zoological Journal of the Linnean Society, 185(3):784-909. https://doi.org/10.1093/zoolinnean/zly068

Mannion, P.D., Upchurch, P., Jin, X., and Zheng, W. 2019b. New information on the Cretaceous sauropod dinosaurs of Zhejiang Province, China: impact on Laurasian titanosauriform phylogeny and biogeography. Royal Society Open Science, 6(8):191057. https://doi.org/10.1098/rsos.191057

Mansfield, J.H. and Abzhanov, A. 2010. Hox expression in the American alligator and evolution of archosaurian axial patterning. Journal of Experimental Zoology Part B: Molecular and Developmental Evolution, 314(8):629-644. https://doi.org/10.1002/jez.b.21364

Marpmann, J.S., Carballido, J.L., Sander, P.M., and Knötschke, N. 2015. Cranial anatomy of the Late Jurassic dwarf sauropod Europasaurus holgeri (Dinosauria, Camarasauromorpha): ontogenetic changes and size dimorphism. Journal of Systematic Palaeontology, 13(3):221263. https://doi.org/10.1080/14772019.2013.875074

Marsh, O.C. 1878. Principal characters of American Jurassic dinosaurs. Part I. American Journal of Science, 16:411-416.

Marsh, A.D. and Rowe, T.B. 2018. Anatomy and systematics of the sauropodomorph Sarahsaurus aurifontanalis from the Early Jurassic Kayenta Formation. PLoS ONE, 13(10):e0204007. https://doi.org/10.1371/journal.pone.0204007

Martínez, R.N. 2009. Adeopapposaurus mognai, gen. et sp. nov. (Dinosauria: Sauropodomorpha), with comments on adaptations of basal Sauropodomorpha. Journal of Vertebrate Paleontology, 29(1):142-164. https://doi.org/10.1671/039.029.0102

Mateus, O., Mannion, P.D., and Upchurch, P. 2014. Zby atlanticus, a new turiasaurian sauropod (Dinosauria, Eusauropoda) from the Late Jurassic of Portugal. Journal of Vertebrate Paleontology, 34(3):618-634. https://doi.org/10.1080/02724634.2013.822875

McIntosh, J.S. and Williams, M.E. 1988. A new species of sauropod dinosaur, Haplocanthosaurus delfsi sp. nov., from the Upper Jurassic Morrison Fm. of Colorado. Kirtlandia, 43:3-26.

Mclntosh, J.S., Miller, W.E., Stadtman, K.L., and Gillette, D.D. 1996. The osteology of Camarasaurus lewisi (Jensen, 1988). Brigham Young University Geology Studies, 41:73-95.

McPhee, B.W., Yates, A.M., Choiniere, J.N., and Abdala, F. 2014. The complete anatomy and phylogenetic relationships of Antetonitrus ingenipes (Sauropodiformes, Dinosauria): implications for the origins of Sauropoda. Zoological Journal of the Linnean Society, 171(1):151-205. https://doi.org/10.1111/zoj.12127

McPhee, B.W., Bonnan, M.F., Yates, A.M., Neveling, J., and Choiniere, J.N. 2015. A new basal sauropod from the pre-Toarcian Jurassic of South Africa: evidence of niche-partitioning at the sauropodomorph-sauropod boundary?. Scientific Reports, 5(1):1-12.

https://doi.org/10.1038/srep13224 
Moore, A.J., Upchurch, P., Barrett, P.M., Clark, J.M., and Xing, X. 2020. Osteology of Klamelisaurus gobiensis (Dinosauria, Eusauropoda) and the evolutionary history of MiddleLate Jurassic Chinese sauropods. Journal of Systematic Palaeontology, 18(16):1299-1393. https://doi.org/10.1080/14772019.2020.1759706

Müller, J., Scheyer, T.M., Head, J.J., Barrett, P.M., Werneburg, I., Ericson, P.G., Pol, D., and Sánchez-Villagra, M.R. 2010. Homeotic effects, somitogenesis and the evolution of vertebral numbers in recent and fossil amniotes. Proceedings of the National Academy of Sciences, 107(5):2118-2123. https://doi.org/10.1073/pnas.0912622107

Nesbitt, S.J. 2011. The early evolution of archosaurs: relationships and the origin of major clades. Bulletin of the American Museum of Natural History, 2011(352):1-292. https://doi.org/10.1206/352.1

Nicholl, C.S.C., Mannion, P.D., and Barrett, P.M. 2018. Sauropod dinosaur remains from a new Early Jurassic locality in the Central High Atlas of Morocco. Acta Palaeontologica Polonica, 63:147-157. https://doi.org/10.4202/app.00425.2017

Osborn, H.F. 1904. Manus, sacrum, and caudals of Sauropoda. Bulletin of the American Museum of Natural History, 20:181-190.

Osborn, H.F. and Mook, C.C. 1921. Camarasaurus, Amphicoelias, and other sauropods of Cope. Memoirs of the AmericanMuseum of Natural History New Series,3:247-387.

Otero, A. and Pol, D. 2013. Postcranial anatomy and phylogenetic relationships of Mussaurus patagonicus (Dinosauria, Sauropodomorpha). Journal of Vertebrate Paleontology, 33(5):1138-1168. https://doi.org/10.1080/02724634.2013.769444

Otero, A., Gallina, P.A., Canale, J.I., and Haluza, A. 2012. Sauropod haemal arches: morphotypes, new classification and phylogenetic aspects. Historical Biology, 24(3):243256. https://doi.org/10.1080/08912963.2011.618269

Otero, A., Krupandan, E., Pol, D., Chinsamy, A., and Choiniere, J. 2015. A new basal sauropodiform from South Africa and the phylogenetic relationships of basal sauropodomorphs. Zoological Journal of the Linnean Society, 174(3):589-634. https://doi.org/10.1111/zoj.12247

Ouyang, H. and Ye, Y. 2002. The First Mamenchisaurian Skeleton with Complete Skull: Mamenchisaurus youngi. Sichuan Science and Technology Press, Chengdu, pp. 111. (In Chinese with English abstract)

Owen, R. 1842. Report on British fossil reptiles, part II. Report of the British Association for the Advancement of Science, 11:60-104.

Parrish, J.M. 2006. The origins of high browsing and the effects of phylogeny and scaling on neck length in sauropodomorphs, p. 201-244. In Carrano, M.T., Gaudin, T.J., Blob, R.W., and Wi, J.R. (eds.), Amniote paleobiology: perspectives on the evolution of mammals, birds and reptiles. The University of Chicago Press, Chicago.

Pi, L., Ou, Y., and Ye, Y. 1996. A new species of sauropod from Zigong, Sichuan, Mamenchisaurus youngi, 87-91. In Department of Spatial Planning and Regional Economy (ed.), Publication in Geoscience Contributed to the 30th International Geological Congress. China Economic Publishing House, Beijing.

Pol, D. and Powell, J. E. 2007. New information on Lessemsaurus sauropoides (Dinosauria: Sauropodomorpha) from the Upper Triassic of Argentina. Special Papers in Palaeontology, 77:223-243.

Pol, D., Garrido, A., and Cerda, I.A. 2011. A new sauropodomorph dinosaur from the Early Jurassic of Patagonia and the origin and evolution of the sauropod-type sacrum. PloSOne, 6:e14572. https://doi.org/10.1371/journal.pone.0014572

Pol, D., Ramezani, J., Gomez, K., Carballido, J.L., Carabajal, A.P., Rauhut, O.W.M., and Cúneo, N.R. 2020. Extinction of herbivorous dinosaurs linked to Early Jurassic global warming event. Proceedings of the Royal Society B, 287(1939):20202310. https://doi.org/10.1098/rspb.2020.2310

Rauhut, O.W. 2003. Revision of Amygdalodon patagonicus Cabrera, 1947 (Dinosauria, Sauropoda). Fossil Record, 6(1):173-181. https://doi.org/10.5194/fr-6-173-2003

Rauhut, O.W.M., Fechner, R., Remes, K., and Reis, K. 2011. How to get big in the mesozoic: the evolution of the sauropodomorph body plan, p. 119-149. In Klein, N., Remes, K., Gee, C.T., Sander, P.M. (eds.), Biology of the Sauropod Dinosaurs: Understanding the life of giants, Indiana University Press. 
Remes, K. 2006. Revision of the Tendaguru sauropod dinosaur Tornieria africana (Fraas) and its relevance for sauropod paleobiogeography. Journal of Vertebrate Paleontology,26(3):651669. https://doi.org/10.1671/0272-4634(2006)26[651:rottsd]2.0.co;2

Remes, K., Ortega, F., Fierro, I., Joger, U., and Kosma, R. 2009. A new basal sauropod dinosaur from the Middle Jurassic of Niger and the early evolution of Sauropoda. PLoS ONE, 4(9):e6924. https://doi.org/10.1371/journal.pone.0006924

Richardson, M.K., Allen, S.P., Wright, G.M., Raynaud, A., and Hanken, J. 1998. Somite number and vertebrate evolution. Development, 125(2):151-160. https://doi.org/10.1242/dev.125.2.151

Royo-Torres, R., Cobos, A., and Alcalá, L. 2006. A giant European dinosaur and a new sauropod clade. Science, 314(5807):1925-1927. https://doi.org/10.1126/science.1132885

Royo-Torres, R., Upchurch, P., Kirkland, J.I., DeBlieux, D.D., Foster, J.R., Cobos, A., and Alcalá, L. 2017. Descendants of the Jurassic turiasaurs from Iberia found refuge in the Early Cretaceous of western USA. Scientific Reports, 7(1):1-12. https://doi.org/10.1038/s41598017-14677-2

Royo-Torres, R., Cobos, A., Mocho, P., and Alcalá, L. 2021. Origin and evolution of turiasaur dinosaurs set by means of a new 'rosetta' specimen from Spain. Zoological Journal of the Linnean Society, 191(1):201-227. https://doi.org/10.1093/zoolinnean/zlaa091

Salgado, L. and Bonaparte, J.F. 1991. Un nuevo saurópodo Dicraeosauridae, Amargasurus cazaui gen. et sp. nov., de la Formación La Amarga, Neocomiano de la provincia del Neuquén, Argentina. Ameghiniana, 28:333-346.

Salgado, L., Coria, R.A., and Calvo, J.O. 1997. Evolution of titanosaurid sauropods: Phytogenetic analysis based on the postcranial evidence. Ameghiniana, 34(1):3-32.

Salgado, L., Carvalho, I.D.S., and Garrido, A.C. 2006. Zapalasaurus bonapartei, a new sauropod dinosaur from La Amarga Formation (Lower Cretaceous), northwestern Patagonia, Neuquén Province, Argentina. Geobios, 39:695-707. https://www.sciencedirect.com/science/article/abs/pii/S0016699506000453

Sander, P.M., Mateus, O., Laven, T., and Knötschke, N. 2006. Bone histology indicates insular dwarfism in a new Late Jurassic sauropod dinosaur. Nature, 441(7094):739-741. https://doi.org/10.1038/nature04633

Sander, P.M., Christian, A., Clauss, M., Fechner, R., Gee, C.T., Griebeler, E.M., and Witzel, U. 2011. Biology of the sauropod dinosaurs: the evolution of gigantism. Biological Reviews, 86(1):117-155. https://doi.org/10.1111/j.1469-185x.2010.00137.x

Schwarz, D., Frey, E., and Meyer, C.A. 2007. Pneumaticity and soft-tissue reconstructions in the neck of diplodocid and dicraeosaurid sauropods. Acta Palaeontologica Polonica, 52(1):167188.

Seeley, H.G.1887. On the classification of the fossil animals commonly called Dinosauria. Proceedings of the Royal Society of London,43:165-171.

Sell, B., Ovtcharova, M., Guex, J., Bartolini, A., Jourdan, F., Spangenberg, J.E., and Schaltegger, U. 2014. Evaluating the temporal link between the Karoo LIP and climatic-biologic events of the Toarcian Stage with high-precision U-Pb geochronology. Earth and Planetary Science Letters, 408:48-56. https://www.sciencedirect.com/science/article/abs/pii/S0012821X14006293

Sellers, K.C., Schmiegelow, A.B., and Holliday, C.M. 2019. The significance of enamel thickness in the teeth of Alligator mississippiensis and its diversity among crocodyliforms. Journal of Zoology, 309(3):172-181. https://doi.org/10.1111/jzo.12707

Sereno, P.C., Beck, A.L., Dutheil, D.B., Larsson, H.C., Lyon, G.H., Moussa, B., and Wilson, J.A. 1999. Cretaceous sauropods from the Sahara and the uneven rate of skeletal evolution among dinosaurs. Science, 286(5443):1342-1347. https://doi.org/10.1126/science.286.5443.1342

Sereno, P.C., Wilson, J.A., Witmer, L.M., Whitlock, J.A., Maga, A., Ide, O., and Rowe, T.A. 2007. Structural extremes in a Cretaceous dinosaur. PloS ONE, 2(11):e1230. https://doi.org/10.1371/journal.pone.0001230

Stumpf, S., Ansorge, J., and Krempien, W. 2015. Gravisaurian sauropod remains from the marine late Early Jurassic (Lower Toarcian) of North-Eastern Germany. Geobios, 48(3):271279. https://doi.org/10.1016/j.geobios.2015.04.001

Taylor, M.P., Wedel, M.J., and Cifelli, R.L. 2011. A new sauropod dinosaur from the Lower Cretaceous Cedar Mountain Formation, Utah, USA. Acta Palaeontologica Polonica, 56(1):75-98. https://doi.org/10.4202/app.2010.0073 
Torcida Fernández-Baldor, F.T., Canudo, J.I., Huerta, P., Montero, D., Suberbiola, X. P., and Salgado, L. 2011. Demandasaurus darwini, a new rebbachisaurid sauropod from the Early Cretaceous of the Iberian Peninsula. Acta Palaeontologica Polonica, 56(3):535-552. https://doi.org/10.4202/app.2010.0003

Tschopp, E., Mateus, O., and Benson, R.B. 2015. A specimen-level phylogenetic analysis and taxonomic revision of Diplodocidae (Dinosauria, Sauropoda). PeerJ, 3:e857. https://doi.org/10.7934/p2124

Tsuihiji, T. 2005. Homologies of the transversospinalis muscles in the anterior presacral region of Sauria (crown Diapsida). Journal of Morphology, 263:151-178. https://doi.org/10.1002/jmor.10294

Upchurch, P. 1994. Sauropod phylogeny and palaeoecology. Gaia, 10:249-260.

Upchurch, P. 1995. The evolutionary history of sauropod dinosaurs. Philosophical Transactions of the Royal Society of London. Series B: Biological Sciences, 349(1330):365-390. https://doi.org/10.1098/rstb.1995.0125

Upchurch, P. 1998. The phylogenetic relationships of sauropod dinosaurs. Zoological Journal of the Linnean Society, 124(1):43-103. https://doi.org/10.1111/j.1096-3642.1998.tb00569.x

Upchurch, P. and Barrett, P.M. 2000. The evolution of sauropod feeding mechanisms, p. 79-122. In Sues, H.D. (ed.), Evolution of Herbivory in Terrestrial Vertebrates: Perspectives from the FossilRecord, Cambridge University. https://doi.org/10.1017/cbo9780511549717.005

Upchurch, P. and Martin, J. 2002. The Rutland Cetiosaurus: the anatomy and relationships of a Middle Jurassic British sauropod dinosaur. Palaeontology, 45(6):1049-1074. https://doi.org/10.1111/1475-4983.00275

Upchurch, P. and Martin, J. 2003. The anatomy and taxonomy of Cetiosaurus (Saurischia, Sauropoda) from the Middle Jurassic of England. Journal of Vertebrate Paleontology, 23(1):208-231. https://doi.org/10.1671/0272-4634(2003)23[208:taatoc]2.0.co;2

Upchurch, P., Barret, P.M., and Dodson, P. 2004. Sauropoda, p. 259-354. In Weishampel, D.P., Dodson, P., and Osmólska, H. (eds.), The Dinosauria, University of California, Berkeley. https://doi.org/10.1525/california/9780520242098.003.0015

Upchurch, P., Mannion, P.D., and Taylor, M.P. 2015. The anatomy and phylogenetic relationships of "Pelorosaurus" becklesii (Neosauropoda, Macronaria) from the Early Cretaceous of England. PloSone, 10(6):e0125819 https://doi.org/10.1371/journal.pone.0125819

Varricchio, D.J. 1997. Growth and embryology. Encyclopedia of Dinosaurs. Academic Press, San Diego, 282-288.

Wang, Y.M., You, H.L., and Wang, T. 2017. A new basal sauropodiform dinosaur from the Lower Jurassic of Yunnan Province, China. Scientific Reports, 7(1):1-11. https://doi.org/10.1038/srep41881

Wedel, M.J. 2003. The evolution of vertebral pneumaticity in sauropod dinosaurs. Journal of Vertebrate Paleontology, 23(2):344-357.

Wedel, M.J. 2005. Postcranial skeletal pneumaticity in sauropods and its implications for mass estimates, p. 201-228. In Curry-Rogers, K.A. and Wilson, J.A. (eds.), The Sauropods: Evolution and Paleobiology, University of California. https://doi.org/10.1525/california/9780520246232.003.0008

Wedel, M.J. 2006. Pneumaticity, neck length, and body size in sauropods. Journal of Vertebrate Paleontology, 26 (3):137-137).

Wedel, M.J. 2007. What pneumaticity tells us about 'prosauropods', and vice versa. Special Papers in Palaeontology, 77:207-222.

Wedel, M.J. 2009. Evidence for bird-like air sacs in saurischian dinosaurs. Journal of Experimental Zoology Part A: Ecological Genetics and Physiology, 311(8):611-628. https://doi.org/10.1002/jez.513

Wedel, M.J., and Sanders, R.K. 2002. Osteological correlates of cervical musculature in Aves and Sauropoda (Dinosauria: Saurischia), with comments on the cervical ribs of Apatosaurus. PaleoBios, 22:1-6.

Wedel, M.J., and Taylor, M.P. 2013. Neural spine bifurcation in sauropod dinosaurs of the Morrison Formation: ontogenetic and phylogenetic implications. PalArch's Journal of Vertebrate Palaeontology, 10(1):1-34.

Wedel, M.J., Cifelli, R.L., and Sanders, R.K. 2000. Osteology, paleobiology, and relationships of the sauropod dinosaur Sauroposeidon. Acta Palaeontologica Polonica, 45(4):343-388. 
Whitlock, J.A. 2011. A phylogenetic analysis of Diplodocoidea (Saurischia: Sauropoda). Zoological Journal of the Linnean Society, 161(4):872915. https://doi.org/10.1111/j.1096-3642.2010.00665.x

Wilson, J.A. 1999. A nomenclature for vertebral laminae in sauropods and other saurischian dinosaurs. Journal of Vertebrate Paleontology, 19(4):639-653. https://doi.org/10.1080/02724634.1999.10011178

Wilson, J.A. 2002. Sauropod dinosaur phylogeny: critique and cladistic analysis. Zoological Journal of the Linnean Society, 136(2):215-275. https://doi.org/10.1046/j.1096-3642.2002.00029.x

Wilson, J.A. and Sereno, P.C. 1998. Early evolution and higher-level phylogeny of sauropod dinosaurs. Journal of Vertebrate Paleontology, 18:1-79. https://doi.org/10.1080/ 02724634.1998 .10011115

Wilson, J.A. and Upchurch, P. 2009. Redescription and reassessment of the phylogenetic affinities of Euhelopus zdanskyi (Dinosauria: Sauropoda) from the Early Cretaceous of China. Journal of Systematic Palaeontology, 7(2):199-239. https://doi.org/10.1017/s1477201908002691

Wilson, J.A., D’Emic, M.D., Ikejiri, T., Moacdieh, E.M., and Whitlock, J.A. 2011. A nomenclature for vertebral fossae in sauropods and other saurischian dinosaurs. PLoS ONE,6:e17114. https://doi.org/10.1371/journal.pone.0017114

Xing, L., Miyashita, T., Currie, P.J., You, H., Zhang, J., and Dong, Z. 2015. A new basal eusauropod from the Middle Jurassic of Yunnan, China, and faunal compositions and transitions of Asian sauropodomorph dinosaurs. Acta Palaeontologica Polonica, 60(1):145154. https://doi.org/10.4202/app.2012.0151

Yates, A.M. 2003. A new species of the primitive dinosaur Thecodontosaurus (Saurischia: Sauropodomorpha) and its implications for the systematics of early dinosaurs. Journal of Systematic Palaeontology, 1(1):1-42. https://doi.org/10.1017/s1477201903001007

Yates, A.M. 2007. The first complete skull of the Triassic dinosaur Melanorosaurus Haughton (Sauropodomorpha: Anchisauria). Evolution and palaeobiology of early sauropodomorph dinosaurs, (77):9-55.

Young, C.C. and Zhao, K.J. 1972. Mamenchisaurus. Memoirs of the Institute of Vertebrate Paleontology and Paleoanthropology, Monograph Series $830 \mathrm{pp}$.

Zhang, Y.H. 1988. The Middle Jurassic dinosaur fauna from Dashanpu, Zigong, Sichuan: sauropod dinosaurs (1) Shunosaurus. Sichuan Publishing House of Science and Technology, Chengdu, China, 3:1-106.

Zhang, Y.H. and Yang, Z.L. 1995. A new complete osteology of Prosauropoda in Lufeng Basin, Yunnan, China: Jingshanosaurus. Yunnan Publishing House of Science and Technology.

Zhang, Q.N., You, H.L., Wang, T., and Chatterjee, S. 2018. A new sauropodiform dinosaur with a 'sauropodan' skull from the Lower Jurassic Lufeng Formation of Yunnan Province, China. Scientific Reports, 8(1):1-12. https://doi.org/10.1038/s41598-018-31874-9 


\section{APPENDIX 1}

Source and measure tables, methodology and results of phylogenetic analysis, character optimizations, character list, and data matrix (available in zipped file for download).

\section{APPENDIX 2}

Data matrix in .nex format (available in zipped file for download). 